\title{
Flora of vascular plants of the Seili island and its surroundings (SW Finland)
}

\author{
Andrzej Brzeg ${ }^{1}$, Wojciech Szwed ${ }^{2} \&$ Maria Wojterska $^{1 *}$
}

${ }^{1}$ Department of Plant Ecology and Environmental Protection, Faculty of Biology, Adam Mickiewicz University in Poznań, Umultowska 89, 61-614 Poznań, Poland

${ }^{2}$ Department of Forest Botany, Faculty of Forestry, Poznań University of Life Sciences, Wojska Polskiego 71D, 60-625 Poznań, Poland

* corresponding author (e-mail: mwzerios@amu.edu.pl; ORCID: https://orcid.org/0000-0002-7774-1419)

\begin{abstract}
The paper shows the results of floristic investigations of 12 islands and several skerries of the inner part of SW Finnish archipelago, situated within a square of $11.56 \mathrm{~km}^{2}$. The research comprised all vascular plants - growing spontaneously and cultivated, and the results were compared to the present flora of a square $10 \times 10 \mathrm{~km}$ from the Atlas of Vascular Plants of Finland, in which the studied area is nested. The total flora counted 611 species, among them, 535 growing spontaneously or escapees from cultivation, and 76 exclusively in cultivation. The results showed that the flora of Seili and adjacent islands was almost as rich in species as that recorded in the square $10 \times 10 \mathrm{~km}$. This study contributed 74 new species to this square. The hitherto published analyses from this area did not focus on origin (geographic-historical groups), socioecological groups, life forms and on the degree of threat of recorded species. Spontaneous flora of the studied area constituted about $44 \%$ of the whole flora of Regio aboënsis. In the studied flora, 22 species threatened in Finland were found.
\end{abstract}

Key words: flora, origin, life forms, socioecological groups, endangered species, distribution patterns, inner archipelago, Turku

\section{Introduction}

The flora of SW Finnish Archipelago was of interest to Eklund (1931, 1946, 1958), whose studies concentrated mainly on the islands of the KorpoHoutskär area and some other islands situated beyond Seili archipelago. The analyses of dynamic tendencies in the flora (based on Eklund's data and on own studies) and patterns of species distribution published by von Numers \& van der Maarel (1998), Korvenpää et al. (2003), Hannus \& von Numers (2008) and von Numers (2011, 2017), did not however cover Seili and adjacent islands. The investigation of the flora of vascular plants of the island Seili and its surroundings was a part of broader studies on the plant cover of this area (Wojterski et al. 1993). Some data on plant taxa, besides Eklund (1946), were given in the karyological work of Arohonka (1982). More recently, vascular plants have been investigated by Leila Linnaluoto, which has resulted in a rich herbarium collection deposited in the Archipelago Research Institute on Seili. Many new floristic data (the lists of species and dates of encounter) were presented in the atlas by Lampinen et al. (2016) and Lampinen (2017).

The aims of this study were to show the richness and differentiation of plant taxa in this part of the SW Finnish Archipelago and comparison to the existing data.

\section{Study area}

Geobotanical studies covered a fragment of archipelago around the island of Seili in SW Finland. The area has been characterised by Wojterska et al. (2018). Documented human influence dates back to the 17 th century, when a leper colony was located on a small island, at that time adjacent to Seili (contemporarily, due to the land uplift, its peninsula), and at least since that time, the main island of Seili has been inhabited. Settlements, roads or piers were present also on three other islands (Kalvgrundet, Katavaluoto and Lammasluoto), the other 16 remained uninhabited. After the death of the last leper patient, a mental hospital was established on Seili. It was closed in 1962, and its 
buildings were adapted in 1977 for the Archipelago Research Institute of the University of Turku. This brought about the cessation of land use, mainly in the valleys that cross the island in the $\mathrm{W}$-E direction, and resulted in serious changes in vegetation. On abandoned fields, different stages of secondary succession were observed, or plantations of trees of different geographic origin, mainly of genera Populus, Picea, Betula, Abies and Pinus, were introduced. Numerous ornamental and useful plants have been cultivated in the proximity of settlements. Nowadays, several years after completion of our investigations, the nature of Seili is protected by the Natura 2000 and Shore Conservation Programmes (Nature on the Island of Seili).

Geobotanical studies were designed and first conducted by the late prof. Teofil Wojterski, through 7 vegetation seasons in the years 1978-1997. The short information on these investigations was published in 1993 (Brzeg et al. 1993; Wojterski et al. 1993).

\section{Material and methods}

The authors' floristic studies, which began in 1978 and were continued in the years 1980, 1981, 1985, 1986, 1996 and 1997, were carried out in the height of the growing season - in July and August, therefore, the early spring flora has been assessed mainly on the basis of herbarium collections of the Archipelago Research Institute of the University of Turku. The investigations focused on the terrestrial flora, and on plants of the littoral zone down to 2 meters depth. The studied area was divided into 44 squares, $0.5 \times 0.5 \mathrm{~km}$ each, nested in the grid present on the topographic map of Finland (Peruskartta 1968). Floristic studies comprised 33 of above mentioned squares (comp. Fig. 6-11). Independently, the detailed distribution of localities of chosen taxa was registered in the form of topograms.

Phytosociological relevés and field observations of all botanists participating in this project (Balcerkiewicz S., Brzeg A., Bujakiewicz A., Kasprowicz M., Szwed W., Wojterska H., Wojterska M. and Wojterski T.) contributed additional information. All the plant species were noted, both wild and cultivated. Over 1300 herbarium sheets with plant specimens were collected for further determination and verification by specialists (see Acknowledgments) and deposited in the herbarium of W. Szwed of the University of Natural Sciences in Poznań. The notes on plant occurrences counted more than 30.000 records.

Table 1. Syntaxonomic definition of socioecological groups of taxa

\begin{tabular}{|c|c|c|}
\hline Category & Syntaxonomic definition & Abbreviation \\
\hline Deciduous forests & Querco-Fagetea, Alnetea glutinosae & $\mathrm{DF}$ \\
\hline Coniferous forests & Vaccinio-Piceetea & $\mathrm{CF}$ \\
\hline Littoral tall herbs & $\begin{array}{l}\text { Bidentetea tripartitae, Cakiletea maritimae, } \\
\text { Filipendulion ulmariae, Phalaridion } \\
\text { arundinaceae, Senecionion fluviatilis (p.p.) }\end{array}$ & LTH \\
\hline Halophytes & $\begin{array}{l}\text { Isoëto durieui-Juncetea bufonii, Juncetea } \\
\text { maritimi, Scirpetum maritimi }\end{array}$ & $\mathrm{H}$ \\
\hline Peats and mires & $\begin{array}{l}\text { Oxycocco-Sphagnetea, Scheuchzerio-Caricetea } \\
\text { fuscae }\end{array}$ & $\mathrm{P}$ \\
\hline Reeds & Phragmitetea (p.max.p.) & $\mathrm{R}$ \\
\hline Water & $\begin{array}{l}\text { Lemnetea, Potametea pectinati, Zosteretea } \\
\text { marinae }\end{array}$ & $\mathrm{W}$ \\
\hline Forest & $\begin{array}{l}\text { Species connected with forest ecosystems, } \\
\text { occurring in different types of forests }\end{array}$ & $\mathrm{F}$ \\
\hline Chasmophytic plants & Asplenietea trichomanis & $\mathrm{C}$ \\
\hline Meadows, pastures \& heathland & $\begin{array}{l}\text { Calluno-Ulicetea (p.max.p.), Molinio- } \\
\text { Arrhenatheretea (p.max.p.) }\end{array}$ & M \\
\hline $\begin{array}{l}\text { Xerothermophilous swards, } \\
\text { forest edges and thickets }\end{array}$ & $\begin{array}{l}\text { Festuco-Brometea, Koelerio-Corynephoretea, } \\
\text { Rhamno-Prunetea, Trifolio-Geranietea } \\
\text { sanguinei }\end{array}$ & $\mathrm{X}$ \\
\hline $\begin{array}{l}\text { Nitrophilous tall herbs and } \\
\text { thickets of forest edges, gaps and } \\
\text { of clear cuttings }\end{array}$ & $\begin{array}{l}\text { Convolvuletalia sepium (p.max.p.), Epilobietea } \\
\text { angustifolii }\end{array}$ & NTH \\
\hline Synanthropic segetal & Aperetalia spicae-venti, Papaveretalia rhoeadis & SS \\
\hline Synanthropic ruderal & $\begin{array}{l}\text { Onopordetalia acanthii, Polygono-Poetea, } \\
\text { Sisymbrietalia }\end{array}$ & SR \\
\hline $\begin{array}{l}\text { Cultivated and escapees from } \\
\text { cultivation }\end{array}$ & - & $\mathrm{C} / \mathrm{SR}$ \\
\hline
\end{tabular}




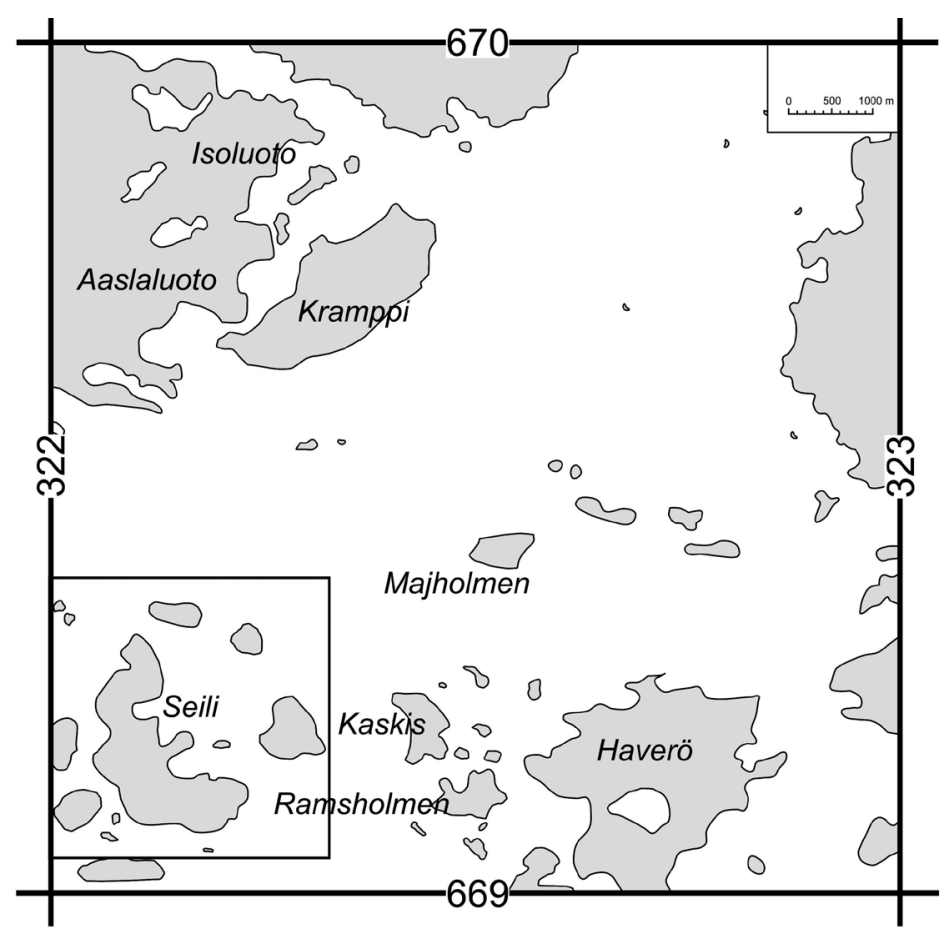

Fig. 1. Square number 669:322 from the Atlas of Distribution of Vascular Plants, grid $10 \times 10 \mathrm{~km}$ (after Lampinen et al. 2016), within which the study area is located

The complete flora of vascular plants was presented in a form of tables (Appendices 1 and 2), containing also information about a square (cf. Fig. 6) in which a given taxon was found.

Division into families was adopted after HämetAhti et al. (1998). The nomenclature and concept of genera, species, and taxa of lower rank were based on the critical compilation of following sources: Pawłowski (1956), Tutin et al. (1964-1980), Lid (1985), Hämet-Ahti et al. (1986, 1998, 2005), Mirek et al. (2002) and Rothmaler et al. (2002). Some species were provided with commonly used synonyms and those used in the Atlas of Distribution of Vascular Plants (Lampinen et al. 2016; Lampinen 2017). Some species, which occurred in the study area in several subspecies or varieties and which identification was impossible, were provided with an abbreviation "s.l.". In all other cases, the determination of subspecies/ varieties means that only those taxa were found during the study.

The lower rank taxa had to be specified, since in the Finnish flora, both in the investigated area and elsewhere in Finland, there are some counterparts with different distribution, ecology or origin, e.g.: Tripleurospermum maritimum ssp. maritimum (native) versus T. maritimum ssp. subpolare (absent in Regio aboënsis, elsewhere an archaeophyte), Cuscuta europaea ssp. europaea (archaeophyte) versus C. europaea ssp. halophyta (native) or Polygonum aviculare ssp. aviculare (archaeophyte, synanthropic ruderal) versus Polygonum aviculare ssp. boreale (native, halophyte). Interpretation of origin or belonging to the socioecological group for such taxa at the level of species is doubtful.

For each taxon (species, subspecies or variety) following data were estimated (Appendix 1):

- origin referred to Regio aboënsis (after Hämet-Ahti et al. 1998, 2005, slightly simplified): $\mathrm{N}$ - native, Aarchaeophyte, EA - established alien; CA - casual alien, found after 1950);

- socioecological group of taxa after concept of van der Maarel (1971), according to the results of own investigations (Table 1); the syntaxonomic diagnoses follow Dierssen \& Dierssen (1996) or Ratyńska et al. (2010);

- main life form according to the concept of Raunkiaer (after Ellenberg et al. 1991, Rothmaler et al. 2002 or Zarzycki et al. 2002);

- local occurrence frequency of taxa counted in 33 squares in the following 5 categories: $\mathrm{c}-$ common (2633 squares), f- frequent (18-25), s-scattered (10-17), $\mathrm{r}$ - rare (3-9), rr - very rare (1-2 squares or stated only in the literature or herbarium and/or not confirmed);

- present in the Atlas of Distribution of Vascular Plants (Lampinen et al. 2016; grid $10 \times 10 \mathrm{~km}$, square number 669:322, within which the study area is located (Fig. 1);

- information about new taxa for the area;

- the category of threat in Finland after Kalliovirta et al. (2010).

Distribution of chosen taxa was given on the background of the generalised map of vegetation complexes (Wojterska et al. 2018). 


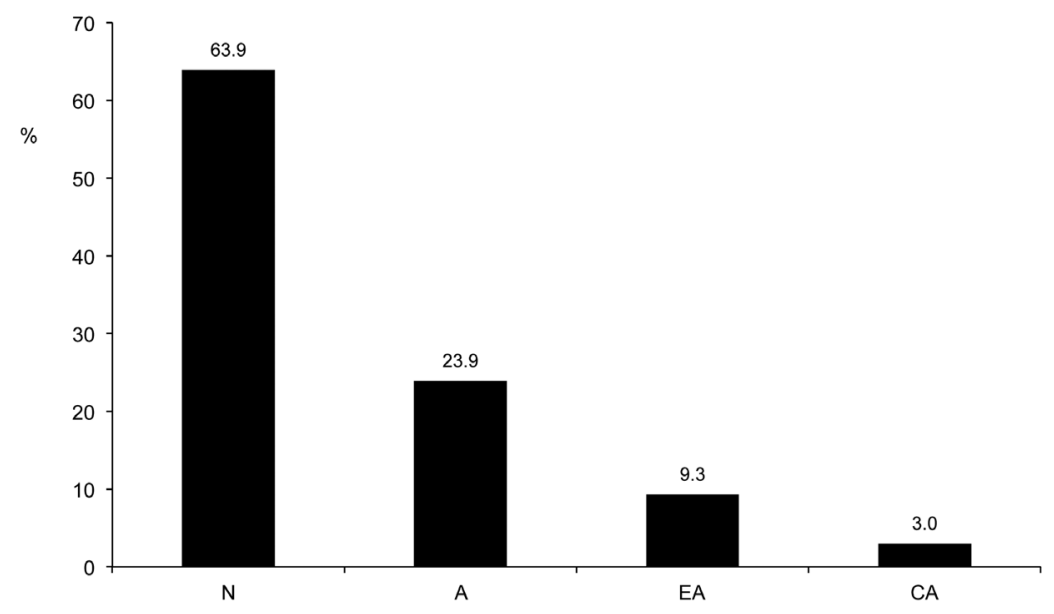

Fig. 2. Spectrum of the origin of taxa, with simplified categories

Explanations: $\mathrm{N}$ - native, $\mathrm{A}$ - archaeophytes, EA - established aliens, CA - casual aliens

\section{Results}

\subsection{Flora of spontaneously occurring plants}

The Appendix 1 includes 540 taxa (535 species) of spontaneously occurring vascular plants: native, effectively naturalised synanthropic and escapees from cultivation. They represented 83 families and 272 genera. The families richest in taxa (Table 2) were: Poaceae ( 52 taxa, $9.7 \%$ of spontaneous flora), Rosaceae $(42,7.8 \%)$ and Asteraceae $(36,6.7 \%)$, whereas 32 families were represented by 1 taxon only.

Carex was the genus richest in taxa (16 taxa, $0.3 \%$ ). Relatively well represented were also: Alchemilla, Hieracium and Polygonum (10 taxa, $0.2 \%$ each). A very big group of genera (166) was represented by only one taxon per genus (Table 3).
Taking into account the spectrum of the origin of taxa (Fig. 2), we found that the most numerous were native taxa $(345-63.9 \%)$. The number of archaeophytes was high $(129-23.9 \%)$, whereas established $(50-9.3 \%)$ and casual $(16-3 \%)$ aliens were less numerous.

The analysis of socioecological groups in the flora of the studied area (Table 1, Fig. 3) has shown that, besides all categories of forest taxa (116) and those connected with littoral $(\mathrm{H}, \mathrm{LTH}$ and $\mathrm{W}-57)$, meadows, pastures and heath (103), also the share of xerothermophilous plants was important (76 taxa). Among natural elements of the flora, the most numerous were taxa connected with deciduous forests and thickets (62), prevailing over the group of coniferous forests taxa (41). Albeit there was a great surface of open rocky habitats (compare Wojterska et al. 2018), the group of chasmophytic vascular plant taxa was not numerous (8). Strictly

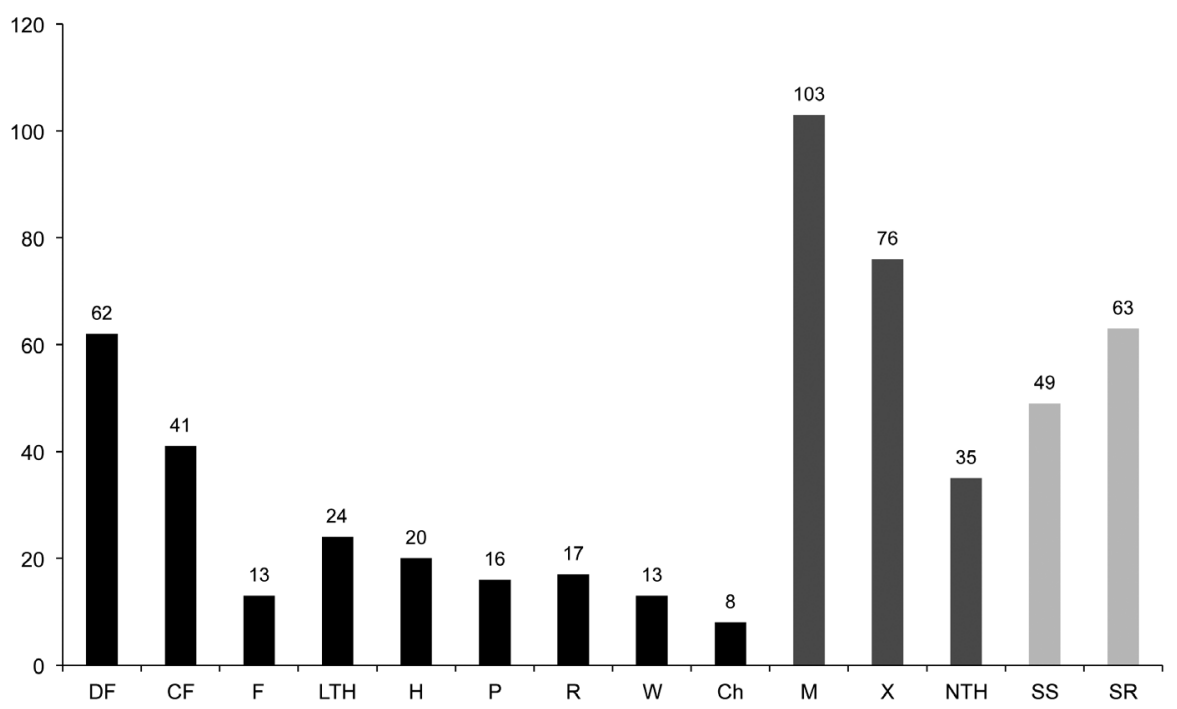

Fig. 3. Spectrum of socioecological groups in the flora of the studied islands Explanations: black colour on the graph - taxa connected with natural or close to natural habitats, dark grey - the plants of seminatural habitats, light grey synanthropic plants. For other abbreviations see Table 1 
Table 2. Floristic richness of families

\begin{tabular}{lcc}
\hline Family & $\begin{array}{c}\text { Number of } \\
\text { taxa in each } \\
\text { family }\end{array}$ & $\%$ \\
\hline Poaceae & 52 & 9.7 \\
Rosaceae & 42 & 7.8 \\
Asteraceae & 36 & 6.7 \\
Caryophyllaceae & 28 & 5.2 \\
Cyperaceae & 23 & 4.3 \\
Cichoriaceae & 22 & 4.1 \\
Brassicaceae & 21 & 3.9 \\
Scrophulariaceae & 20 & 3.7 \\
Fabaceae, Lamiaceae & 18 & 3.3 \\
Polygonaceae, Ranunculaceae & 17 & 3.1 \\
Apiaceae, Juncaceae & 12 & 2.2 \\
Boraginaceae, Dryopteridaceae, Pyrolaceae, Rubiaceae & 9 & 1.7 \\
Onagraceae, Orchidaceae, Primulaceae & 8 & 1.5 \\
Chenopodiaceae, Ericaceae, Salicaceae, Violaceae & 7 & 1.3 \\
Betulaceae, Campanulaceae, Equisetaceae, Plantaginaceae & 5 & 0.9 \\
Alliaceae, Convallariaceae, Crassulaceae, Grossulariaceae, Lycopodiaceae, & 4 & 0.7 \\
Potamogetonaceae & & \\
Aspleniaceae, Caprifoliaceae, Geraniaceae, Solanaceae & 3 & 0.6 \\
Clusiaceae, Convolvulaceae, Euphorbiaceae, Fumariaceae, Gentianaceae, & 2 & 0.4 \\
Juncaginaceae, Liliaceae, Malvaceae, Papaveraceae, Pinaceae, Typhaceae, & & \\
Urticaceae, Valerianaceae & & \\
Aceraceae, Adoxaceae, Asclepiadaceae, Asparagaceae, Callitrichaceae, & 1 & 0.2 \\
Cupressaceae, Cuscutaceae, Eleagnaceae, Empetraceae, Fagaceae, & & \\
Haloragaceae, Hippuridaceae, Hypolepidaceae, Iridaceae, Lemnaceae, & & \\
Linaceae, Lythraceae, Menyanthaceae, Oleaceae, Ophioglossaceae, & \\
Oxalidaceae, Polypodiaceae, Portulacaceae, Saxifragaceae, Sparganiaceae, & & \\
Thelypteridaceae, Tiliaceae, Trilliaceae, Ulmaceae, Vitaceae, Zannichelliaceae, & & \\
Zosteraceae & & \\
Total & 540 & \\
\hline
\end{tabular}

Table 3. Floristic richness of genera

Name or number of genera

Number of

taxa in each

\begin{tabular}{lc} 
& genus \\
\hline Carex & 16 \\
Alchemilla, Hieracium, Polygonum & 10 \\
Galium, Veronica & 9 \\
Juncus, Poa, Rosa & 8 \\
Epilobium, Vicia, Viola & 7 \\
Agrostis, Festuca, Myosotis, Ranunculus, Salix & 6 \\
Calamagrostis, Campanula, Dryopteris, Equisetum, Plantago, Pyrola, Rumex, & 5 \\
Stellaria, Trifolium & \\
Allium, Cerastium, Chenopodium, Cirsium, Eleocharis, Lathyrus, Luzula, & 4 \\
Lysimachia, Potamogeton, Potentilla, Ribes, Sedum, Sonchus & \\
Achillea, Asplenium, Atriplex, Galeopsis, Geranium, Lamium, Melampyrum, & 3 \\
Melandrium, Rubus, Senecio, Silene, Sorbus, Taraxacum, Vaccinium & \\
Alnus, Alopecurus, Anemone, Angelica, Anthemis, Arabis, Arctium, & \\
Artemisia, Aster, Barbarea, Betula, Cardamine, Centaurea, Centaurium, & \\
Cotoneaster, Deschampsia, Elymus, Eriophorum, Euphorbia, Euphrasia, & \\
Fallopia, Filipendula, Fragaria, Geum, Gnaphalium, Hypericum, Listera, & \\
Lolium, Lycopodium, Malva, Matricaria, Mentha, Odontites, Pimpinella, & \\
Polygonatum, Raphanus, Sagina, Scleranthus, Scutellaria, Solanum, Spergula, & \\
Spergularia, Thlaspi, Triglochin, Tripleurospermum, Typha, Urtica, Valeriana & \\
166 genera & \\
Total & \\
& \\
&
\end{tabular}




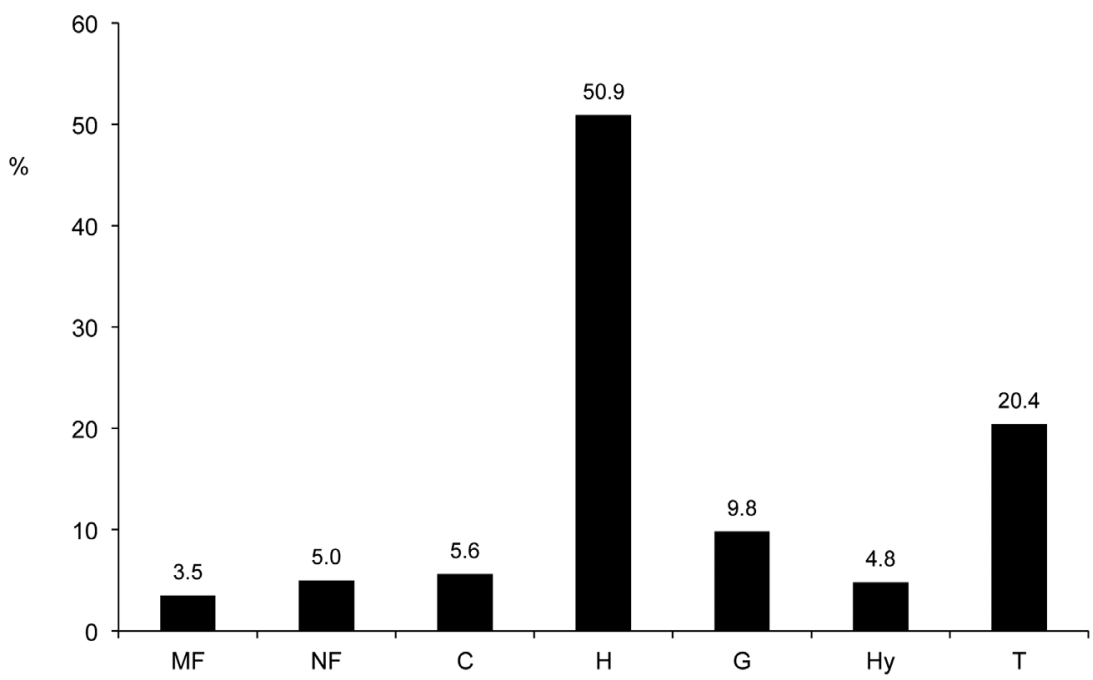

Fig. 4. Life forms spectrum in the flora of the studied islands

Explanations: MF - megaphanerophytes, NF - nanophanerophytes, C - chamaephytes, H-hemicryptophytes, G-geophytes, Hy-hydrophytes, T-therophytes

synanthropic taxa (segetal and ruderal) were also numerous (112). Taxa connected with natural or close to natural habitats (214), were less numerous, than those occurring on anthropogenically transformed sites (326) (Fig. 3). In the last group, the number of plants of seminatural habitats (214) was higher than that of synanthropic (112) (Fig. 3).

In the life form spectrum, hemicryptophytes constituted over $50 \%$ (Fig. 4). They were followed by therophytes with $20.4 \%$. The lowest was the share of megaphanerophytes - only $3.5 \%$.

\subsection{Frequency of occurrence and distribution patterns of species}

Rare and very rare taxa constituted $53.5 \%$ of the list, whereas the group of most common, found in more than 25 squares, comprised less than $10 \%$ of the total list (Fig. 5). Number of taxa found in one square varied from 41 (D1) to 393 (E3) (Appendix 1).

In all or almost all squares $(0.5 \times 0.5 \mathrm{~km})$, there were noted halophilous taxa, such as: Juncus gerardii (33), Agrostis stolonifera ssp. maritima (33), and Glaux maritima (31), those of littoral reeds and tall herbs group: Lythrum salicaria (33) and Phalaris arundinacea (33), hydrophytes: Batrachium baudotii (33), Zannichellia palustris (32), and Potamogeton perfoliatus (32), as well as of coniferous forests: Juniperus communis (31), Deschampsia flexuosa (31), and Pinus sylvestris (31).

The group of species that were noted only in one square counted 38 (cf. Appendix 1); out of them, some occurred only at single localities e.g.: Artemisia

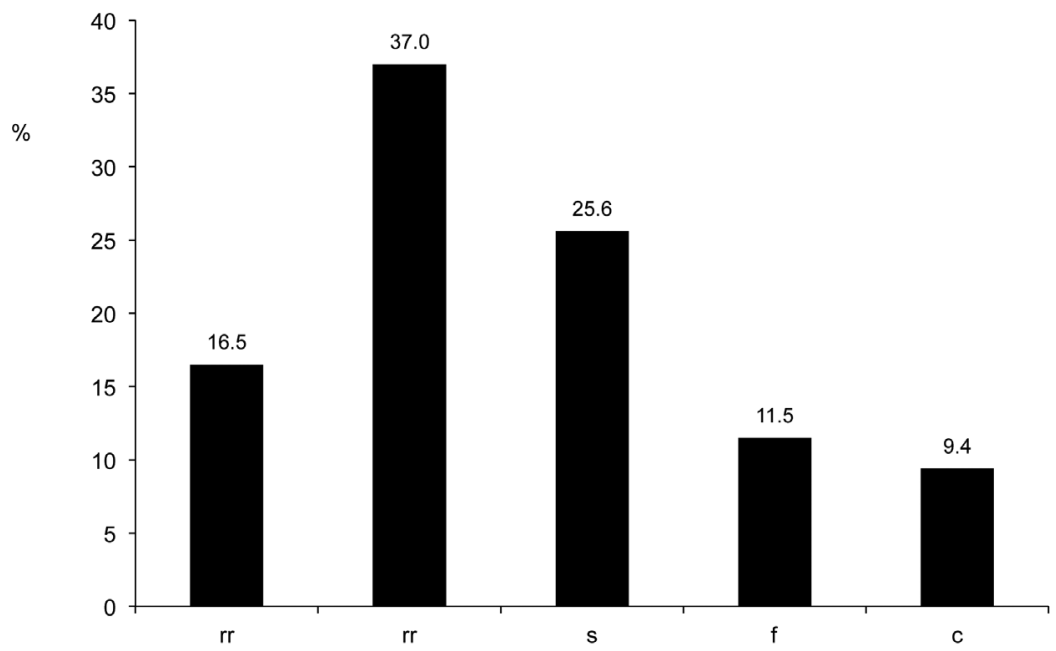

Fig. 5. The share of frequency groups in the whole flora Explanations: $\mathrm{rr}-$ very rare, $\mathrm{r}-$ rare, $\mathrm{s}-$ scattered, $\mathrm{f}-$ frequent, $\mathrm{c}-$ common 

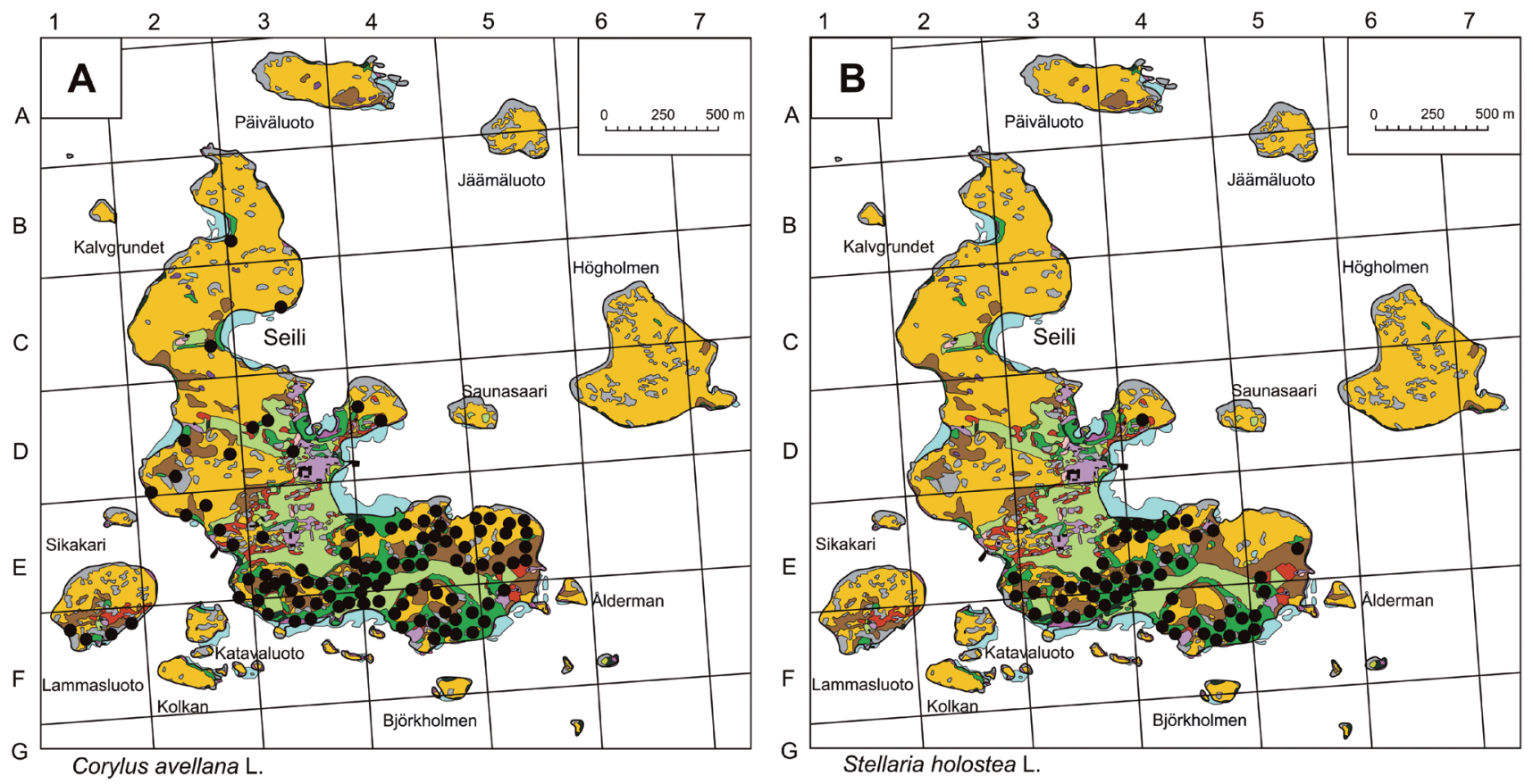

Fig. 6. Distribution of selected indicator species of deciduous forests Explanations: A - Corylus avellana, B - Stellaria holostea

absinthium, Callitriche palustris, Centaurea cyanus, Corallorhiza trifida, Eleocharis parvula, Hyoscyamus niger, Juncus bulbosus, Malva pusilla, Melandrium noctiflorum, Rubus chamaemorus, Silene viscosa, and Vicia sylvatica.

Distribution of chosen species, depicted in the form of topograms, shows patterns characteristic for some spatial vegetation complexes. Distribution of Corylus avellana and Stellaria holostea (Fig. 6A-B) was in accordance with the complex of eutrophic deciduous forests and thickets, whereas that of Goodyera repens, Ledum palustre and Listera cordata (Fig. 7A-B) with oligotrophic pine and spruce forests. Asplenium septentrionale and Woodsia ilvensis (Fig. 8A) were
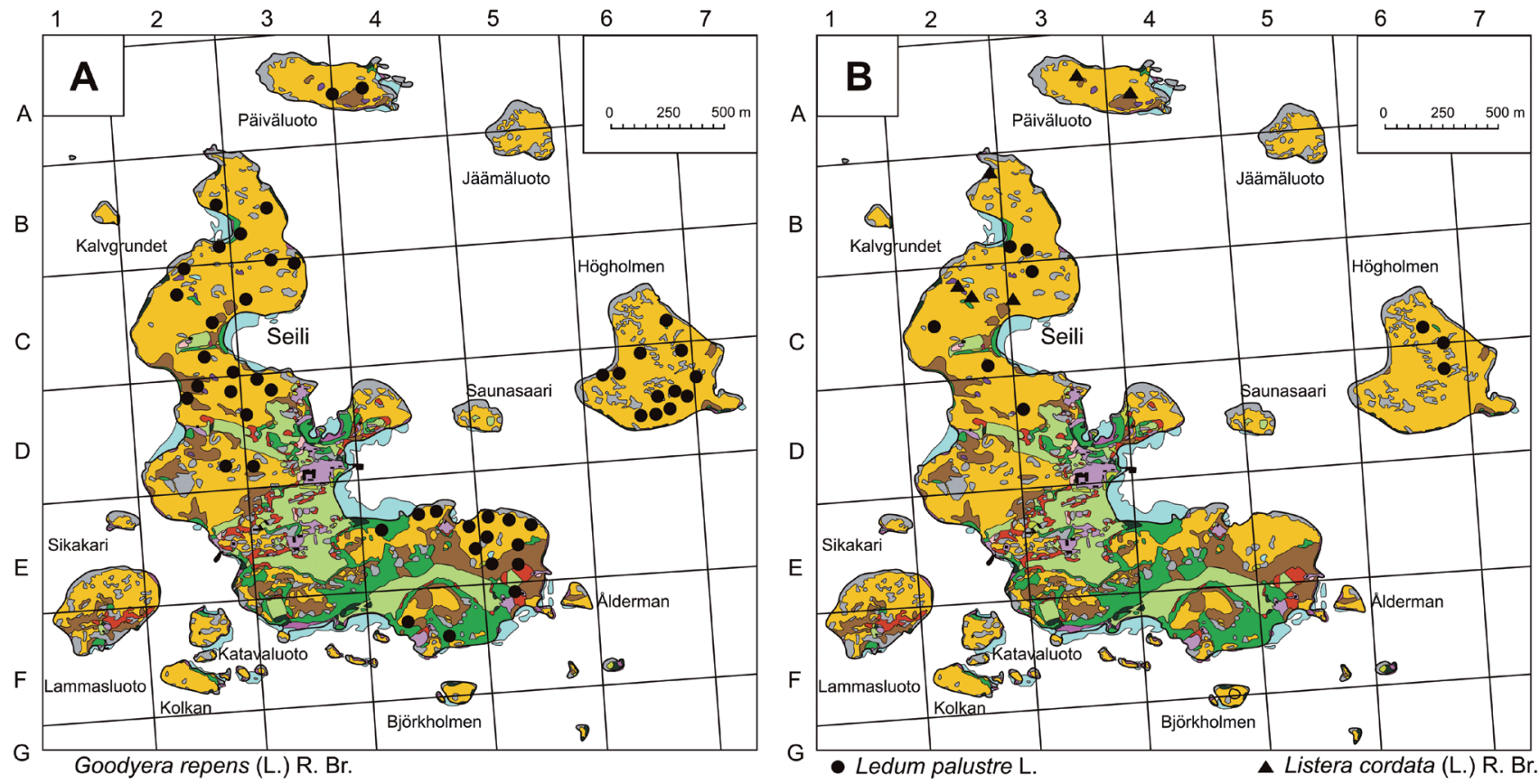

Fig. 7. Distribution of selected indicator species of coniferous forests Explanations: A - Goodyera repens, B - Ledum palustre, Listera cordata 

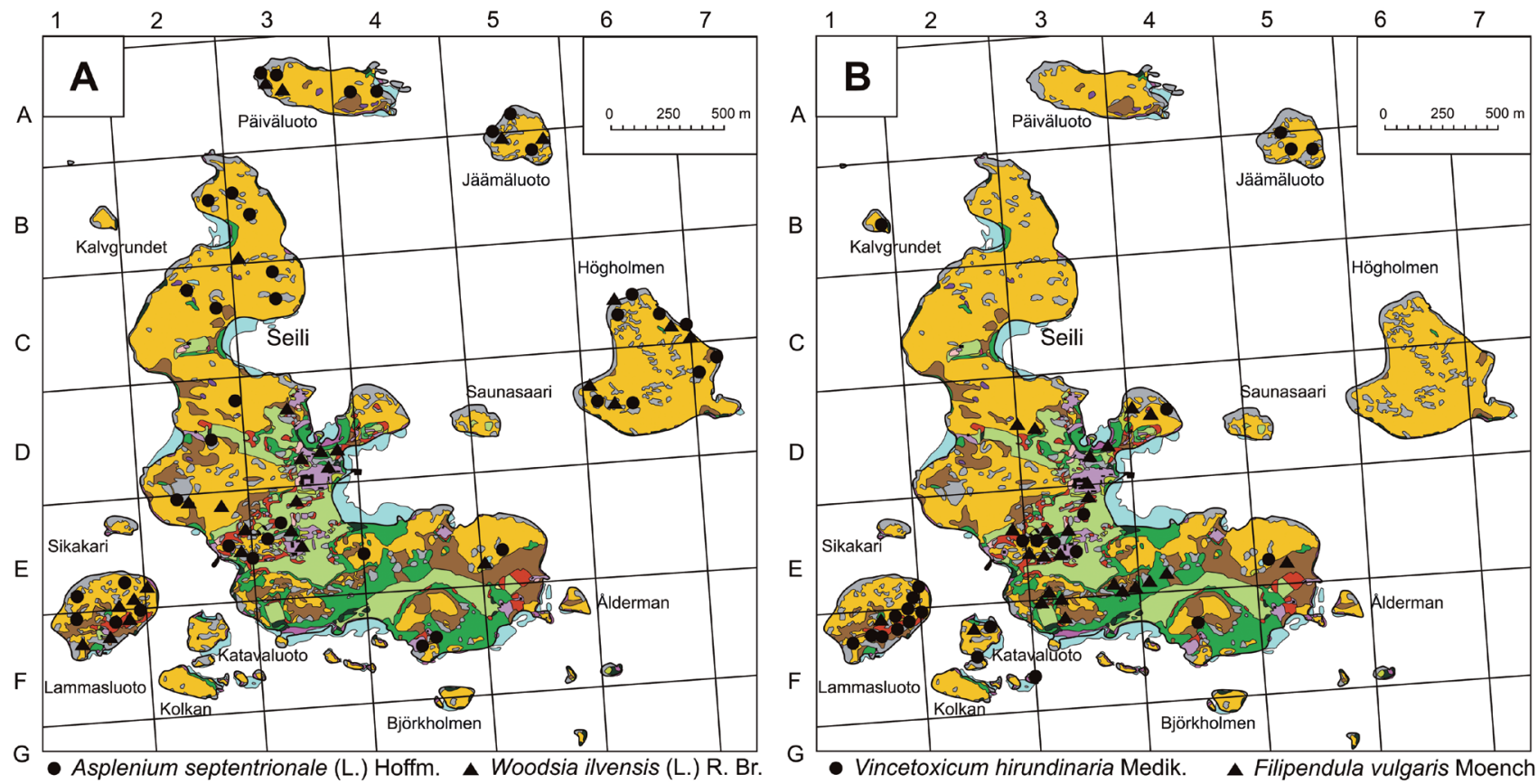

Fig. 8. Distribution of selected indicator species

Explanations: A - epilithic communities, Asplenium septentrionale and Woodsia ilvensis; B - xerothermophilous forest edges, Vincetoxicum hirundinaria and Filipendula vulgaris

indicators of the complex of epilithic communities, Vincetoxicum hirundinaria and Filipendula vulgaris (Fig. 8B) for the complex of xerothermophilous swards, forest edges and thickets. Juncus gerardii (Fig. 9A) occurred along all flat coasts, similarly like the complex of coastal halophytes, whereas Juncus compressus (Fig. 9B), connected with extensively used roads, within the complex of seminatural meadows and pastures. Distribution of Arctium minus and Leonurus cardiaca (Fig. 10A-B) was in accordance with the complex of synanthropic ruderal group, found mainly in the proximity of settlements. Littoral hydrophytes were represented by Batrachium baudotii and Zannichellia palustris (Fig. 11A-B).
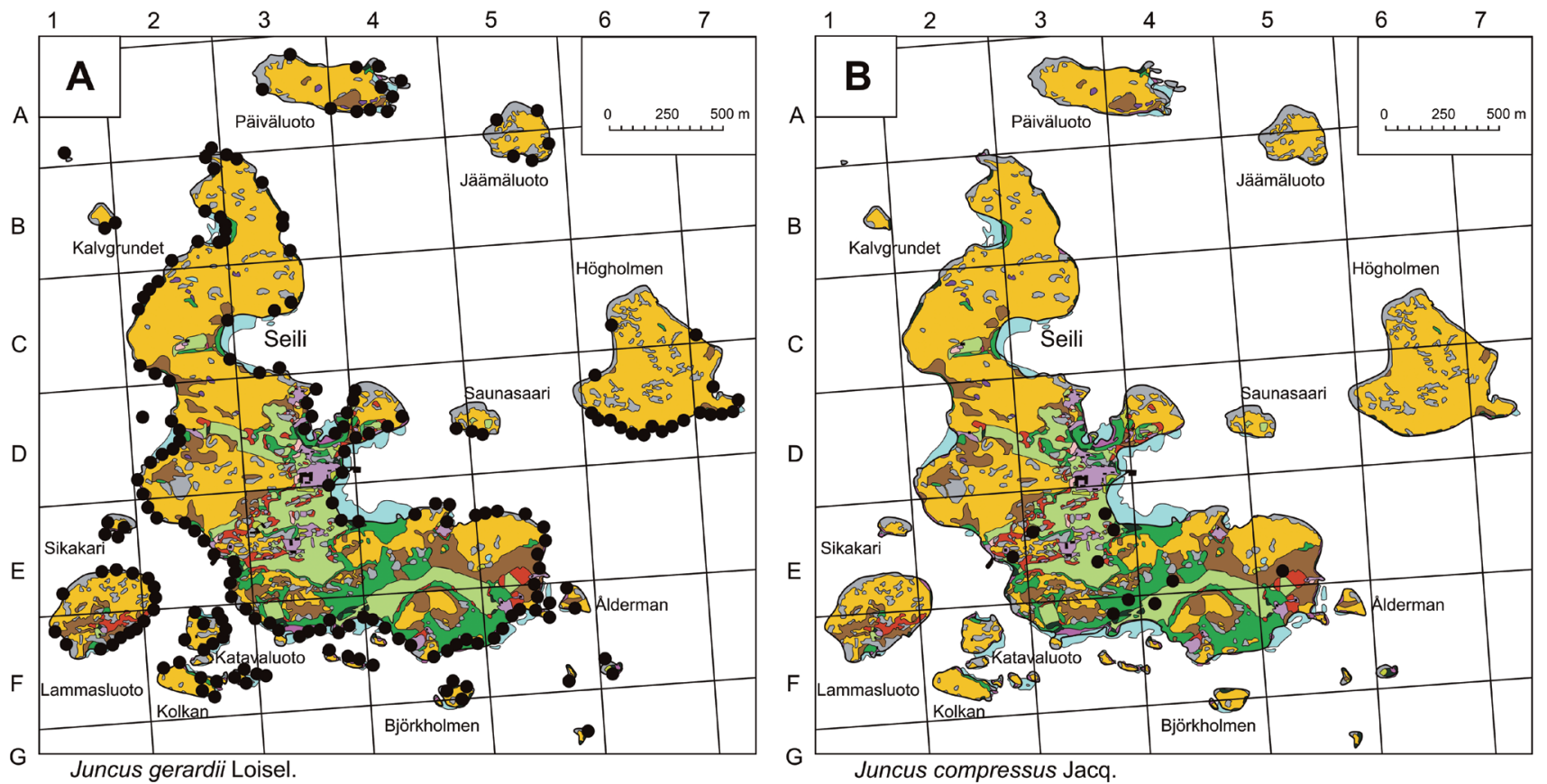

Fig. 9. Distribution of selected indicator species

Explanations: A - coastal halophytes, Juncus gerardii; B - seminatural meadows and pastures, Juncus compressus 

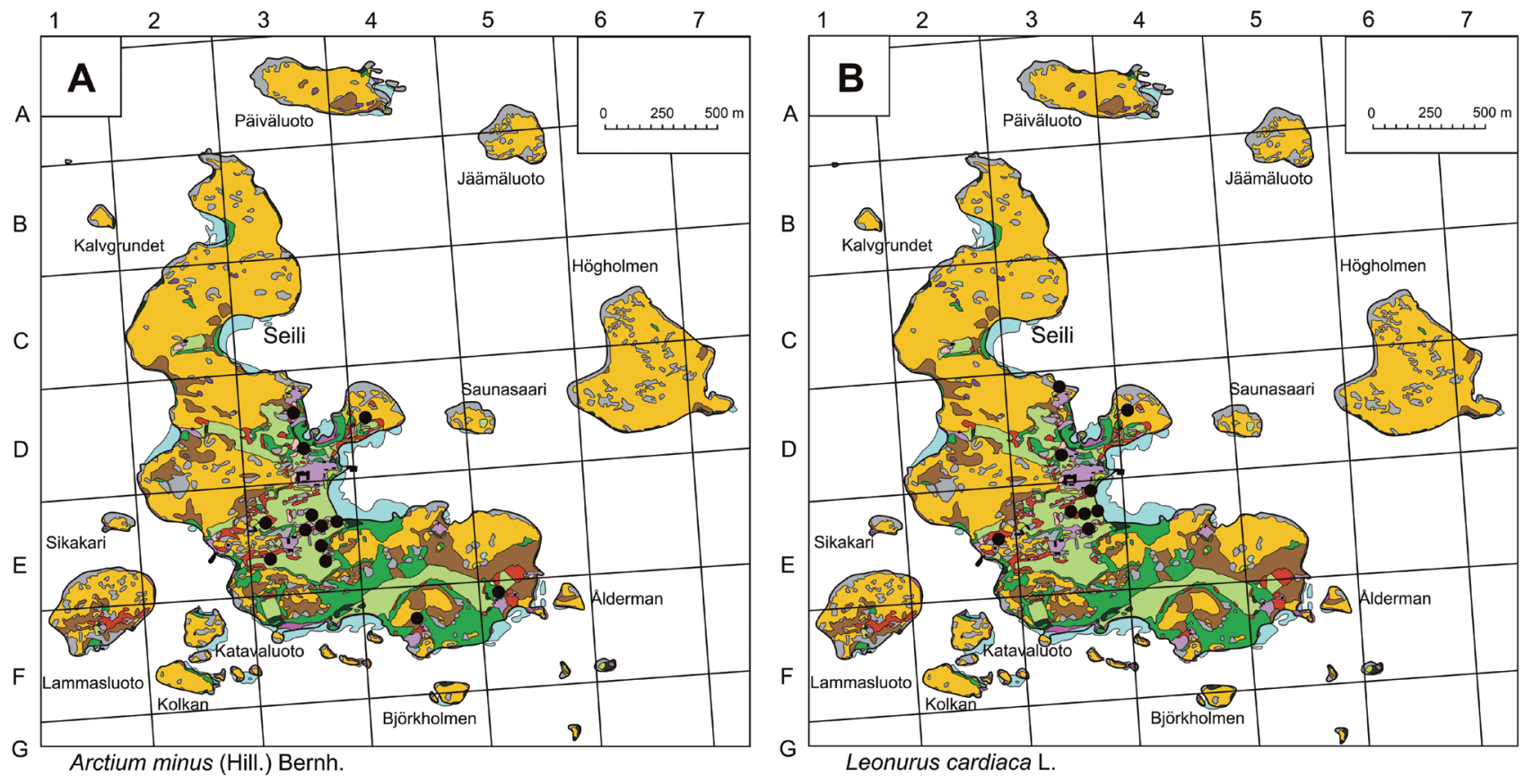

Fig. 10. Distribution of selected indicator species of ruderal communities Explanations: A - Arctium minus, B - Leonurus cardiaca

\subsection{Flora of cultivated plants}

The second list (Appendix 2), encompasses 76 cultivated or introduced plants growing in fields, gardens and tree plantations. During the period of study, these plants were not found in the wild. They were noted in 14 out of 33 studied squares. They represented 32 families. with the following being richest in species: Pinaceae
(10), Asteraceae (7), Rosaceae (7) and Apiaceae (5). Among the families of cultivates plants, 7 were not represented in the wild: Amaryllidaceae, Cucurbitaceae, Hyacinthiaceae, Hydrangeaceae, Paeoniaceae, Rutaceae and Taxaceae. There were found 61 genera. The genus Picea (6) was the richest in species, followed by: Abies (3) and Betula (3), all from different geographic regions,
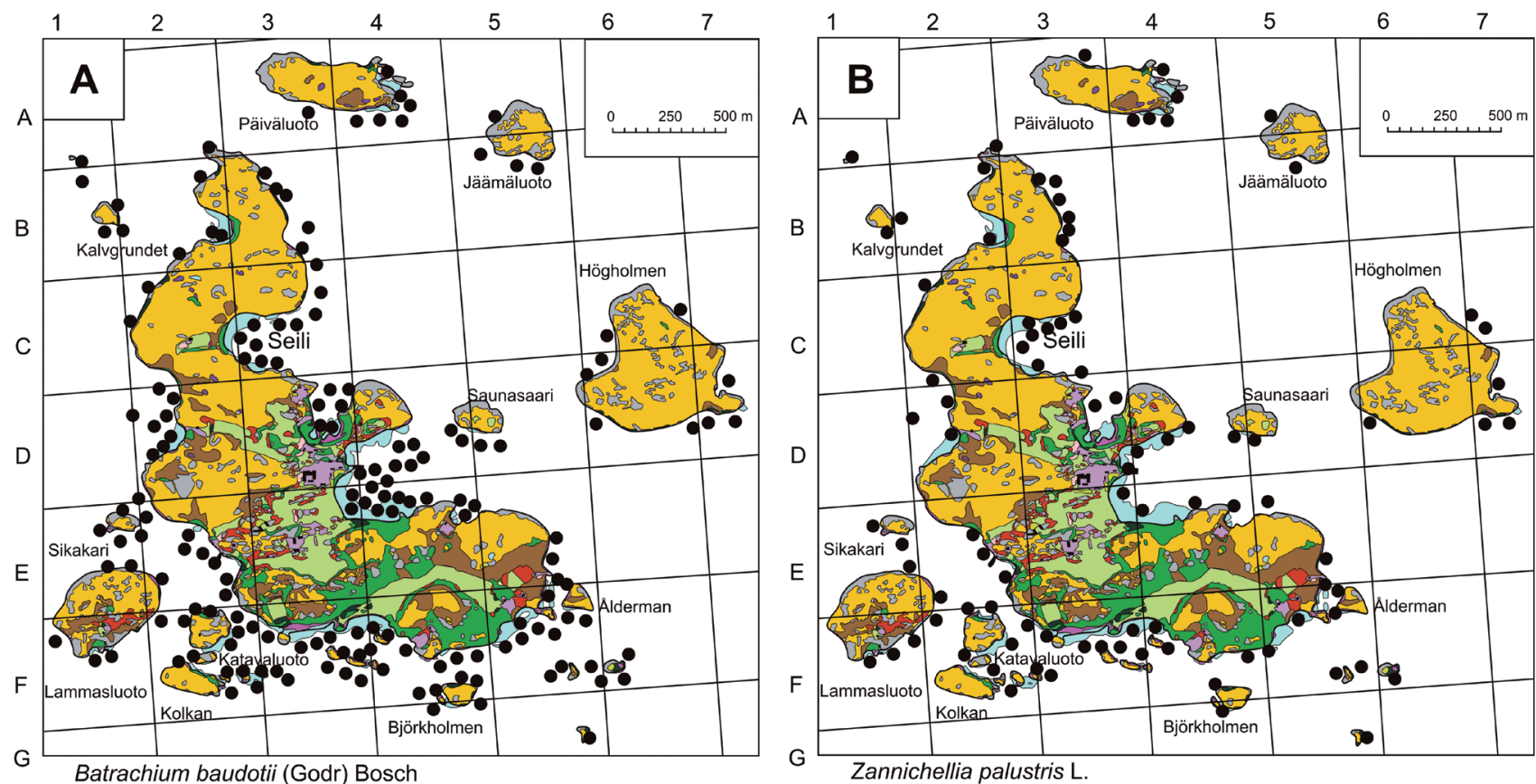

Fig. 11. Distribution of selected indicator species of littoral hydrophytes Explanations: A - Batrachium baudotii, B - Zannichellia palustris 
introduced in a form of plantations on abandoned fields. Allium (3), Brassica (2), Cerasus (2), Hemerocallis (2) and Syringa (2) were represented by three or two species, whereas the remaining 53 genera by only one species.

The life form spectrum showed the prevalence of phanerophytes (MF - 20, NF - 9) over hemicryptophytes (14). The second group were therophytes (15). Cultivated plants were relatively less frequent, they all belonged to the category rare (21) or very rare (55), because the most frequent Syringa vulgaris was found only in 8 squares.

\section{Discussion}

During our botanical explorations, 11 spontaneously occurring species reported by Eklund (1946), Arohonka (1982) or collected by Leila Linnaluoto (not publ.) were not found. These were: Anthemis arvensis, Berteroa incana, Cardamine hirsuta, Convolvulus arvensis, Hieracium suecicum, Hippuris vulgaris, Lithospermum arvense, Lolium multiflorum, Montia fontana, Sparganium minimum and Thlaspi caerulescens.

Eklund has contributed 280 species of spontaneously occurring vascular plants to the flora of Seili (Appendix 1), Arohonka-261, while in the herbarium of Archipelago Research Institute there were 216 species. Altogether there were 409 spontaneously occurring taxa known from Seili. Our study supplemented this list with 131 taxa.

Data concerning distribution of the following early spring species: Adoxa moschatellina, Anemone ranunculoides, Corydalis solida, Ficaria verna, Gagea minima, Muscari botryoides, Ornithogalum angustifolium and Scilla siberica, might be incomplete due to the lack of our investigations in spring.

The present work has contributed 76 new, spontaneously occurring taxa, which previously were not noted in the square number 669:322 (compare Lampinen et al. 2016), and 46 found only in cultivation. Some of them were quite frequent, e.g.: Alchemilla sarmatica (noted in 14 squares), Festuca salina (30), Hieracium linifolium (15), H. murorum (12), Rumex tenuifolius (27), Taraxacum balticum (14), and T. officinale (21).

Out of 540 species found in the above mentioned square (669:322), 96 were not found on Seili and adjacent islands, although, some of them were quite widespread in a big square, including: Alisma plantagoaquatica, Alliaria petiolata, Artemisia campestris, Bolboschoenus maritimus, Carex muricata, $C$. pseudocyperus, Frangula alnus, Lemna minor and Potentilla neglecta (whereas $P$. argentea s.str. was noted in 14 squares on Seili). Lack of some freshwater plants was connected with the observed diminishing or even disappearance of water bodies.
Some plants mentioned in the list of Lampinen (1.c.) probably got extinct in the discussed square, concluding from the date of the last record. On the other hand, 13 species noted only before the WWII were confirmed on Seili during our study (Alchemilla filicaulis, Callitriche palustris, Carex viridula, Euphrasia stricta, Heracleum sphondylium s.l., Lathyrus linifolius, Lycopodium clavatum, Moneses uniflora, Myosotis stricta, Pyrola rotundifolia, Festuca arundinacea, Urtica urens, Vicia angustifolia).

The high share of archaeophytes in the studied archipelago resulted first of all from the local concept of this group of species (which included e.g. the great part of meadow plants) and from high share of hemerochorous species in the Finnish flora, which has been indicated and discussed already by Erkamo (1959, 1961), Jalas (1961, 1965), Sukopp (1972), or Suominen and Hämet-Ahti (1993). On the other hand, a low share of established, and, especially, casual aliens was conspicuous.

Comparison of the spontaneous flora of the studied area, with the flora of Regio aboënsis $\left(11500 \mathrm{~km}^{2}-1215\right.$ species, Lahti et al. 1988) showed that $44 \%$ of all species found in this region occurred in the Seili archipelago.

According to our observations in the period of almost 20 years, the dynamics of populations (number of localities and abundance of individuals) of following 50 species of vascular plants had shown the tendency to decrease and they may be endangered if this tendency continues: Alchemilla acutiloba, A. glabra, A. xanthochlora, Anagallis arvensis, Arctium minus, A. tomentosum, Arabis hirsuta, Artemisia absinthium, Asperugo procumbens, Asplenium viride, Callitriche palustris, Campanula patula, Carduus crispus, Carex elata, Centaurea cyanus, Cerastium glomeratum, Chenopodium glaucum, Ch. polyspermum, Corallorhiza trifida, Dactylorhiza maculata, Dentaria bulbifera, Descurainia sophia, Diphasiastrum complanatum, Eleocharis parvula, Epilobium lamyi, E. obscurum, Fumaria officinalis, Galium × pomeranicum, Hippophaë rhamnoides, Hyoscyamus niger, Hypochoeris maculata, Juncus bulbosus, Lathyrus linifolius, L. niger, Leonurus cardiaca, Malva pusilla, Matricaria chamomilla, Melandrium album, M. noctiflorum, Papaver somniferum, Ranunculus bulbosus, Salix phylicifolia, Scleranthus annuus, Silene viscosa, Sisymbrium officinale, Solanum nigrum, Sorbus intermedia, Ulmus glabra, Urtica urens and Vicia sylvatica. The measures that have been undertaken in the last decades, such as: mowing, prescribed burning and cattle grazing, may contribute to the restoration of the traditional cultural landscape and preservation of the island's biodiversity (Nature of the Island of Seili).

23 species from the red list of plant species in Finland (Kalliovirta et al. 2010) were found on the 
islands: 3 critically endangered - CR (Pimpinella major, Polygonum oxyspermum, Rosa canina s.str.), 3 endangered - EN (Epilobium lamyi, E. obscurum, Lithospermum arvense), 5 vulnerable - VU (Cirsium oleraceum, Galium verum, Melampyrum arvense, Sorbus intermedia, Ulmus glabra), 11 near threatened - NT (Alchemilla plicata, A. propinqua, Antennaria dioica, Cardamine pratensis, Centaurium pulchellum, Chimaphila umbellata, Dianthus deltoides, Nardus stricta, Pyrola media, Taxus baccata, Zostera marina) and one of data deficient - DD (Rosa corymbifera). One of the above mentioned taxa was found only in culture (Taxus baccata). Some of these red list species were quite abundant, especially on Seili, e.g.: Galium verum, Melampyrum arvense, Rosa canina and Zostera marina. Their resources were not locally endangered, provided that the extensive way of management would be maintained.

Although the studied area covers only $11.56 \mathrm{~km}^{2}$, it shows striking floristic richness. The stated number of spontaneously occurring species (535) is similar to that of the $10 \times 10 \mathrm{~km}$ square 669:322 (540 species, among them 40 not confirmed after WWII, Lampinen et al. 2016). The high number of new species (76) can be partly explained by slightly narrower concept of some species, and by taking into account escapees from cultivation in the studied flora. It is worth mentioning, however, that both the Finnish flora (Hämet-Ahti et al. 1998, 2005) and Atlas of Vascular Plants (Lampinen et al. 2016) also comprise several cultivated species. List of cultivated species (Appendix 2) gives opportunity for the assessment of their behaviour in future, i.e., their tendencies for spreading in the wild.

In the last decades, papers addressing the dynamics of flora of the Archipelago were published (von Numers \& van der Maarel 1998; Korvenpää et al. 2003; Hannus \& von Numers 2008; von Numers 2011, 2017) taking as a benchmark the flora of Eklund (1958). Our study comprised some not yet analysed areas and aspects, such as the share of threatened species, importance of cultivated species for the total floristic richness and their local dynamics.

\section{Conclusions}

Considering the rather small area $\left(11.56 \mathrm{~km}^{2}\right)$ of Seili islands such high number of plant species (535) confirms the importance of this area for the protection of the richness of flora and its representativeness for the whole region.

Intensive, detailed studies conducted on a relatively small area may contribute importantly to the enrichment of the inventory of species in a region.

Acknowledgments. The studies on Seili were possible due to the financial support of the Archipelago Research Institute of the University of Turku and from the Polish Committee of Scientific Research (Grant 6 P04C 022 10). The geobotanical exploration on Seili has been performed by listed earlier group of researchers. Several of them made their notes and herbarium accessible and by doing so helped in investigating this flora. Also in the determination of some taxa other botanists were of great help. The following groups of species were identified or verified by: Maria Pawlus Alchemilla and Festuca; Karol Latowski - Brassicaceae and Apiaceae; Waldemar Żukowski - Cyperaceae, especially Eleocharis; Jerzy Zieliński - Abies, Picea, Rosa and Rubus. We hereby express our gratitude for the favour and time they have granted us. Special thanks are due to Fiona MilneRostkowska, who made linguistic corrections.

\section{Author Contributions}

Research concept and design: A. Brzeg, W. Szwed Acquisition and/or assembly of data: A. Brzeg, W. Szwed, M. Wojterska

Data analysis and interpretation: A. Brzeg, M. Wojterska Drafting the article: A. Brzeg, W. Szwed, M. Wojterska Critical revision: A. Brzeg, M. Wojterska

Final approval: A. Brzeg, M. Wojterska

\section{References}

Arohonka T. 1982. Kromosomiluku Määrityksiä Nauvon Seilin saaren putkilokasveista (Chromosome counts of vascular plants of the island Seili in Nauvo, SW Finland). Turun Yliop. Biol. Laitos. Julk. 3: 1-12.

Brzeg A,. Bujakiewicz A., Rusińska A., Szwed W., Tobolewski Z. \& Wojterski T. 1993. Flora of the island Seili in southwestern Finnland. Wiad. Bot. 37(3/4): 21-23 (in Polish with English Summary).

Dierssen K. \& Dierssen B. 1996. Vegetation Nordeuropas. 838 pp. E. Ulmer Verlag, Stuttgart.

EKLUND O. 1931. Über die Ursachen der regionalen Verteilung der Schärenflora SW-Finlands. Acta Botanica Fennica 8: $1-133$.
EKLUND O. 1946. Über die Kalkabhängigkeit der Kormophyten SW-Finland. Memoranda Soc. Fauna et Flora Fennica 22: 166-187.

EkLuND O. 1958. Die Gefässpflanzenflora beiderseits Skiftet im Schärenarchipel Südwestfinnlands: Kirchspiele, Korpo, Houtskär, Nagu, Inio, Brändö, Kumlinge, Sottunga und Kökar. Bidrag till K nnedom af Finlands. Natur och Folk 101: 1-321.

Ellenberg H., Weber H. E., Dull R., Wirth V., Werner W. \& Paulisen D. 1991. Zeigerwerte von Pflanzen in Mitteleuropa [Indicator values of plants in Central Europe]. 248 pp. Scripta Geobotanica. 18. Verlag Erich Goltze KG, Göttingen. 
ERKAMO V. 1959. Über die Zahlenverhältnisse der synanthropen und der ursprünglichen Pflanzenarten Finnlands. Arch. Soc. Vanamo 13: 132-140.

Erкамо V. 1961. Über die Synanthropen in der finnischen Flora. Fennia 85: 82-85.

Hämet-Ahti L., Suominen J., Ulvinen T., Uotila P. \& Vuокко S. 1986. Retkeilykasvio. 598 pp. Suomen Luonnonsuojelun Tutki Oy, Forssa.

Hämet-Ahti L., Suominen J., Ulvinen T. \& Uotila P. (eds.). 1998. Retkeilykasvio. (Field Flora of Finnland), Ed. 4. 656 pp. Finnish Museum of Natural History, Botanical Museum, Helsinki.

Hämet-Ahti L, Kurtto A., Lampinen R., Piirainen M., Suominen J., Ulvinen T., Uotila P. \& VÄre H. 2005. Lisäyksiä ja korjauksia Retkeilykasvion neljänteen painokseen. Lutukka 21: 41-85.

HANNus J.-J. \& VON NuMERs M. 2008. Vascular plant richness in relation to habitat diversity and island area in the Finnish Archipelago. J Biogeogr 35: 1077-1086.

JALAS J. 1961. Fälle von Introgression in der Flora Finnlands hervorgerufen durch die Tätigkeit des Menschen. Fennia 85: 58-81.

JALAS J. 1965. Hemerobe und hemerochore Pflanzenarten. Ein terminologischer Reformversuch. Acta Soc. Fauna Flora Fennica 72(2): 1-15.

Kalliovirta M., Ryttäri T., HÆggström, C.-A., Hakalisto S., Kanerva T., Koistinen M., Lammi A., Lehtelä M., Rautiainen V.-P., Rintanen T., Salonen V. \& Uusitalo A. 2010. Putkilokasvit. Vascular Plants. Tracheophyta. In: P. RASSI, E. HyväRINEN, A. JUSLÉN \& I. Mannerkoski (eds.). Suomen lajien uhanalaisuus Punainen kirja (The 2010 Red List of Finnish Species), pp. 183-203. Ministry of the Environment, Finnish Environment Institute, Helsinki.

KorvenpäÄ T., von Numers M. \& Hinneri S. 2003. A mesoscale analysis of floristic patterns in the SW Finnish Archipelago. J Biogeogr 30: 1019-1031.

Lahti T., Kurtto A. \& VÄIsÄnen R. 1988. Floristic composition and regional species richness of vascular plants in Finland. Ann Bot Fenn 25: 281-291.

LAMPINEN R. 2017. Kasviatlas 2016: Tilastokarttoja. http:// www.luomus.fi/kasviatlas/tilasto/atlas2016_tila stokartat.pdf. (access 8.12.2017).

Lampinen R., Lahti T., Kurtto A. \& Lommi S. 2016. Atlas of the Distribution of Finnish Vascular Plants (Kasviatlas, Växtatlas). Square 669:322. http://www.luomus. fi/kasviatlas (access 8.12.2017).

LID J. 1985. Norsk, Svensk, Finsk Flora. 5 ed. 837 pp. Det Norske Samlaget, Oslo.

MaArel E. van Der 1971. Florastatistieken als bijdrage tot de evaluatie van ntuurgebieden. Gorteria 5: 176-188.

Mirek Z., Piękoś-Mirkowa H., Zając A. \& ZająC M. 2002. Flowering plants and pteridophytes of Poland. A checklist. In: Z. MIREK (ed.). Biodiversity of Poland, 1, 442 pp. W. Szafer Institute of Botany, Polish Academy of Sciences, Kraków.
NATURE on the Island of Seili http://www.nationalparks.fi/ seili/nature Access: 27.12. 2018

PawŁowski B. 1956. Flora Tatrorum. Plantae vasculares 1. 672 pp. PWN, Warszawa.

PeruskartTa 1:20 000 1968. 1043 04 Aaslaluoto. (Topographic map of Finnland). Maanmittaushallituksen Topografinen Toimisto. Helsinki.

PetäJä A. \& Juusti T. 1979. Archipelago Research Institute. $14 \mathrm{pp}$. University of Turku, Turku.

Ratyńska H., Wojterska M., Brzeg A. \& KoŁacz M. 2010. Multimedialna encyklopedia zbiorowisk roślinnych Polski ver. 1.1. Instytut Edukacyjnych Technologii Informatycznych. Uniwersytet Kazimierza Wielkiego, Bydgoszcz.

Rothmaler W., Jäger E. J. \& Werner K. 2002. Exkursionsflora von Deutschland. 4. Gefäßpflanzen: Kritischer Band. 948 pp. Spektrum Akad. Verl., Heidelberg, Berlin.

Sukopp H. 1972. Wandel von Flora und Vegetation in Mitteleuropa unter dem Einfluß des Menschen. Berichte über Landwirtschaft 50(1): 112-139.

SuOMinen J. \& HÄMET-Aнті L. 1993. Kasvistomme muinaistulokkaat: tulkintaa ja perusteluja. (Archaeophytes in the flora of Finnland). Norrlnia 4: 1-90.

Tutin T. G., Heywood V. H., Burges N. A., Moore D. M., Valentine D. H., Walters S. M., Webb D. A. (eds.). 1964-1980. Flora Europaea. 1-5. Cambridge University Press, Cambridge.

VON NuMERS M. 2011. Sea shore plants of the SW archipelago of Finnland - distribution patterns and long term changes in $20^{\text {th }}$ century. Ann Bot Fenn 48 (Suppl. A): $1-46$.

VON Numers M. 2017. Distribution patterns and long term changes in vascular plants of non-littoral areas in SW archipelago of Finnland. Part I. Study concept and Pteridophyta. Ann Bot Fenn 54: 245-262.

VON NuMERS M. \& VAN DER MAAREL E. 1998. Plant distribution patterns and ecological gradients in the SouthwestFinnish archipelago. Global Ecology and Biogegraphy Letters 7:421-440.

Wojterska M., Balcerkiewicz S. \& Brzeg A. 2018. Map of vegetation complexes of the Seili island and its surroundings (SW Finland). Biodiv. Res. Conserv. 51: $35-41$

Wojterski T., Balcerkiewicz S., Brzeg A., Bujakiewicz A., Kasprowicz M., Łuszczyński J. Rusińska A., Szwed W., Tobolewski Z., Wojterska H. \& Wojterska M. 1993. Vegetation of the island Seili in southwestern Finnland. Wiad. Bot. 37(3/4):17-19 (in Polish with English Summary).

Zarzycki K., Trzcińska-Tacik H., Różański W., Szeląg Z., WoŁeK J. \& KorZeniaK U. 2002. Ecological indicator values of vascular plants of Poland. In: Z. MireK (ed.). Biodiversity of Poland, 2, 183 pp. W. Szafer Institute of Botany, Polish Academy of Sciences, Kraków. 
Appendices 
Appendix 1. Specification and characteristics of spontaneous vascular flora of Seili archipelago

\begin{tabular}{|c|c|c|c|c|c|c|c|c|c|c|c|}
\hline 1 & 2 & 3 & 4 & 5 & 67 & $78 \quad 9$ & 10 & 11 & 12 & 13 & 14 \\
\hline & Square & & & & & & $\mathrm{A}_{1}$ & $\mathrm{~A}_{2}$ & $\mathrm{~A}_{3}$ & $\mathrm{~A}_{4}$ & $\overline{\mathrm{A}_{5}}$ \\
\hline 1 & Acer platanoides L. & $\mathrm{N}$ & DF & MF & 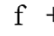 & + & & & + & + & \\
\hline 2 & Achillea collina Becker ex Rchb. & A & $\mathrm{X}$ & $\mathrm{H}$ & r & & & & & & \\
\hline 3 & Achillea millefolium L. ssp. millefolium & A & M & $\mathrm{H}$ & 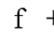 & + & & & + & + & \\
\hline 4 & Achillea ptarmica $\mathrm{L}$. & EA & M & $\mathrm{H}$ & $f+$ & + & & + & + & + & \\
\hline 5 & Acinos arvensis (Lam.) Dandy [Satureja acinos (L.) Scheele] & $\mathrm{N}$ & $\mathrm{X}$ & $\mathrm{T}$ & $r+$ & + & & & & & \\
\hline 6 & Aconitum $\times$ stoerkianum $\mathrm{Rhb}$. & $\mathrm{CA}$ & $\mathrm{C} / \mathrm{SR}$ & $\mathrm{H}$ & $\mathrm{r}$ & + & & & & & \\
\hline 7 & Actaea spicata $\mathrm{L}$. & $\mathrm{N}$ & $\mathrm{DF}$ & $\mathrm{H}$ & $\mathbf{r}+$ & + & & & & & \\
\hline 8 & Adoxa moschatellina $\mathrm{L}$. & $\mathrm{N}$ & DF & $\mathrm{G}$ & $\mathbf{r}+$ & + & & & & & \\
\hline 9 & Aegopodium podagraria $\mathrm{L}$. & $\mathrm{N}$ & NTH & $\mathrm{H}$ & $\mathrm{r}+$ & + & & & & & \\
\hline 10 & Aethusa cynapium L. & A & SS & $\mathrm{T}$ & $\mathbf{r}+$ & + & & & & & \\
\hline 11 & Agrimonia eupatoria $\mathrm{L}$. & $\mathrm{N}$ & $\mathrm{x}$ & $\mathrm{H}$ & $\mathrm{r}+$ & + & & & & & \\
\hline 12 & Agrostis canina L. & $\mathrm{N}$ & $\mathrm{P}$ & $\mathrm{H}$ & $s-$ & + & & & + & + & \\
\hline 13 & Agrostis capillaris L. [A. tenuis Sibth.] & A & M & $\mathrm{H}$ & $f+$ & + & & + & + & + & \\
\hline 14 & Agrostis gigantea Roth & $\mathrm{N}$ & M & $\mathrm{H}$ & $r+$ & + & & & & & \\
\hline 15 & Agrostis stolonifera ssp. maritima (Lam.) G.Mey. & $\mathrm{N}$ & $\mathrm{H}$ & $\mathrm{H}$ & $c+$ & + & + & + & + & + & + \\
\hline & Agrostis stolonifera ssp. prorepens (K. Koch) Asch. & $\mathrm{N}$ & M & $\mathrm{H}$ & $s+$ & + & & & & & \\
\hline 16 & Agrostis vinealis Schreb. & $\mathrm{N}$ & $\mathrm{X}$ & $\mathrm{H}$ & $\mathrm{rr}+$ & + & & & & & + \\
\hline 17 & Ajuga reptans $\mathrm{L}$. & $\mathrm{CA}$ & $\mathrm{C} / \mathrm{SR}$ & $\mathrm{H}$ & $\mathbf{r}$ & + & & & & & \\
\hline 18 & Alchemilla acutiloba Opiz [A. vulgaris L. ssp. acutangula (Buser) Murb.] & A & M & $\mathrm{H}$ & $\mathrm{rr}+$ & + & & & & & \\
\hline 19 & Alchemilla fillicaulis Buser var. fillicaulis & EA & M & $\mathrm{H}$ & $\mathrm{r}$ & & & & & & \\
\hline 20 & Alchemilla glabra Neygenf. & $\mathrm{N}$ & M & $\mathrm{H}$ & $\mathrm{rr}$ & + & & & & & \\
\hline 21 & Alchemilla micans Buser [A. gracilis Opiz] & A & M & $\mathrm{H}$ & s & + & & & & & \\
\hline 22 & Alchemilla monticola Opiz & A & M & $\mathrm{H}$ & $\mathrm{s}+$ & + & & & & & \\
\hline 23 & Alchemilla plicata Buser & A & M & $\mathrm{H}$ & $\mathrm{r}$ & NT & & & & & \\
\hline 24 & Alchemilla propinqua $\mathrm{H}$. Lindb. ex Juz. & $\mathrm{EA}$ & M & $\mathrm{H}$ & r & + NT & & & & & \\
\hline 25 & Alchemilla sarmatica Juz. & EA & M & $\mathrm{H}$ & s & + & & & & & \\
\hline 26 & Alchemilla subcrenata Buser & A & M & $\mathrm{H}$ & $\mathrm{r}+$ & + & & & & & \\
\hline 27 & Alchemilla xanthochlora Rothm. & $\mathrm{EA}$ & M & $\mathrm{H}$ & $\mathrm{r}$ & + & & & & & \\
\hline 28 & Allium oleraceum L. & A & $\mathrm{x}$ & $\mathrm{G}$ & $\mathbf{r}$ & + & & & & & \\
\hline 29 & Allium schoenoprasum L. ssp. schoenoprasum & $\mathrm{N}$ & $\mathrm{x}$ & $\mathrm{G}$ & $\mathrm{f}+$ & + & & + & + & + & + \\
\hline 30 & Allium scorodoprasum L. & $\mathrm{A}$ & M & $\mathrm{G}$ & $\mathbf{r}+$ & + & & & & & \\
\hline 31 & Allium vineale $\mathrm{L}$. & $\mathrm{A}$ & $\mathrm{x}$ & $\mathrm{G}$ & $\mathrm{r}$ & + & & & & & \\
\hline 32 & Alnus glutinosa (L.) Gaertn. & $\mathrm{N}$ & $\mathrm{DF}$ & MF & $\mathrm{c}+$ & + & & + & + & + & + \\
\hline 33 & Alnus incana (L.) Moench ssp. incana & $\mathrm{N}$ & $\mathrm{DF}$ & MF & $\mathrm{rr}$ & + & & & & & \\
\hline 34 & Alopecurus geniculatus L. & A & M & $\mathrm{H}$ & $\mathrm{r}$ & + & & & & + & \\
\hline 35 & Alopecurus pratensis $\mathrm{L}$. & $\mathrm{A}$ & M & $\mathrm{H}$ & $s+$ & + & & & & & \\
\hline 36 & Anagallis arvensis $\mathrm{L}$. & $\mathrm{CA}$ & SS & $\mathrm{T}$ & $\mathbf{r}$ & + & & & & & \\
\hline 37 & Anchusa arvensis (L.) M. Bieb. & A & SS & $\mathrm{T}$ & $r+$ & + & & & & & \\
\hline 38 & Anemone nemorosa $\mathrm{L}$. & $\mathrm{N}$ & $\mathrm{DF}$ & $\mathrm{G}$ & $s+$ & + & & & + & + & \\
\hline 39 & Anemone ranunculoides $\mathrm{L}$. & $\mathrm{N}$ & $\mathrm{DF}$ & $\mathrm{G}$ & $r+$ & + & & & & & \\
\hline 40 & Anethum graveolens $\mathrm{L}$. & $\mathrm{CA}$ & $\mathrm{C} / \mathrm{SR}$ & $\mathrm{T}$ & $\mathbf{r}$ & + & & & & & \\
\hline 41 & Angelica archangelica L. ssp. litoratis (Fr.) Thell. & $\mathrm{N}$ & LTH & $\mathrm{H}$ & $c+$ & + & + & + & + & + & + \\
\hline 42 & Angelica sylvestris L. & $\mathrm{N}$ & M & $\mathrm{H}$ & $c+$ & + & & + & + & + & + \\
\hline 43 & Antennaria dioica (L.) Gaertn. & $\mathrm{N}$ & M & $\mathrm{C}$ & s & NT & & & + & & \\
\hline 44 & Anthemis arvensis $\mathbf{L}$. & A & SS & $\mathrm{T}$ & $\mathrm{rr}+$ & + & & & & & \\
\hline 45 & Anthemis tinctoria L. [Cota tinctoria (L.) J. Gay.] & EA & $\mathrm{X}$ & $\mathrm{H}$ & $\mathrm{rr}$ & + & & & & & \\
\hline 46 & Anthoxanthum odoratum L. ssp. odoratum & $\mathrm{N}$ & M & $\mathrm{H}$ & $s+$ & + & & & & & \\
\hline 47 & Anthriscus sylvestris (L.) Hoffm. & A & NTH & $\mathrm{H}$ & $\mathrm{f}+$ & + & & + & & + & \\
\hline 48 & Aquilegia vulgaris L. & EA & $\mathrm{C} / \mathrm{SR}$ & $\mathrm{H}$ & r & + & & & & & \\
\hline 49 & Arabidopsis thatiana (L.) Heynh. & $\mathrm{N}$ & $\mathrm{x}$ & $\mathrm{T}$ & s & + & & & & & \\
\hline 50 & Arabis glabra (L.) Bernh. [Turritis glabra L.] & $\mathrm{N}$ & $X$ & $\mathrm{H}$ & $r+$ & + & & & & & \\
\hline 51 & Arabis hirsuta (L.) Scop. & $\mathrm{N}$ & $\mathrm{X}$ & $\mathrm{H}$ & $\mathbf{r}$ & + & & & & & \\
\hline 52 & Arctium minus Bernh. & A & SR & $\mathrm{H}$ & $\mathrm{r}+$ & + & & & & & \\
\hline 53 & Arctium tomentosum Mill. & $\mathrm{A}$ & SR & $\mathrm{H}$ & $\mathrm{r}$ & + & & & & & \\
\hline 54 & Arctostaphylos uva-ursi (L.) Spreng. & $\mathrm{N}$ & $\mathrm{CF}$ & $\mathrm{Ch}$ & $f+$ & + & & & + & + & + \\
\hline 55 & Arenaria serpyllifolia $\mathrm{L}$. & $\mathrm{N}$ & $\mathrm{x}$ & $\mathrm{T}$ & $r+$ & + & & & & & \\
\hline 56 & Armoracia rusticana P. Gaertn., B. Mey. et Scherb. & EA & $\mathrm{C} / \mathrm{SR}$ & $\mathrm{H}$ & $\mathrm{rr}$ & + & & & & & \\
\hline 57 & Artemisia absinthium L. & EA & SR & $\mathrm{Ch}$ & $\mathrm{rr}$ & + & & & & & \\
\hline 58 & Artemisia vulgaris L. var. vulgaris & A & SR & $\mathrm{H}$ & $s+$ & + & & & & & \\
\hline 59 & Asparagus officinalis L. & EA & $\mathrm{C} / \mathrm{SR}$ & G & $\mathrm{rr}$ & + & & & & & \\
\hline
\end{tabular}


Biodiv. Res. Conserv. 53: 33-65, 2019

47

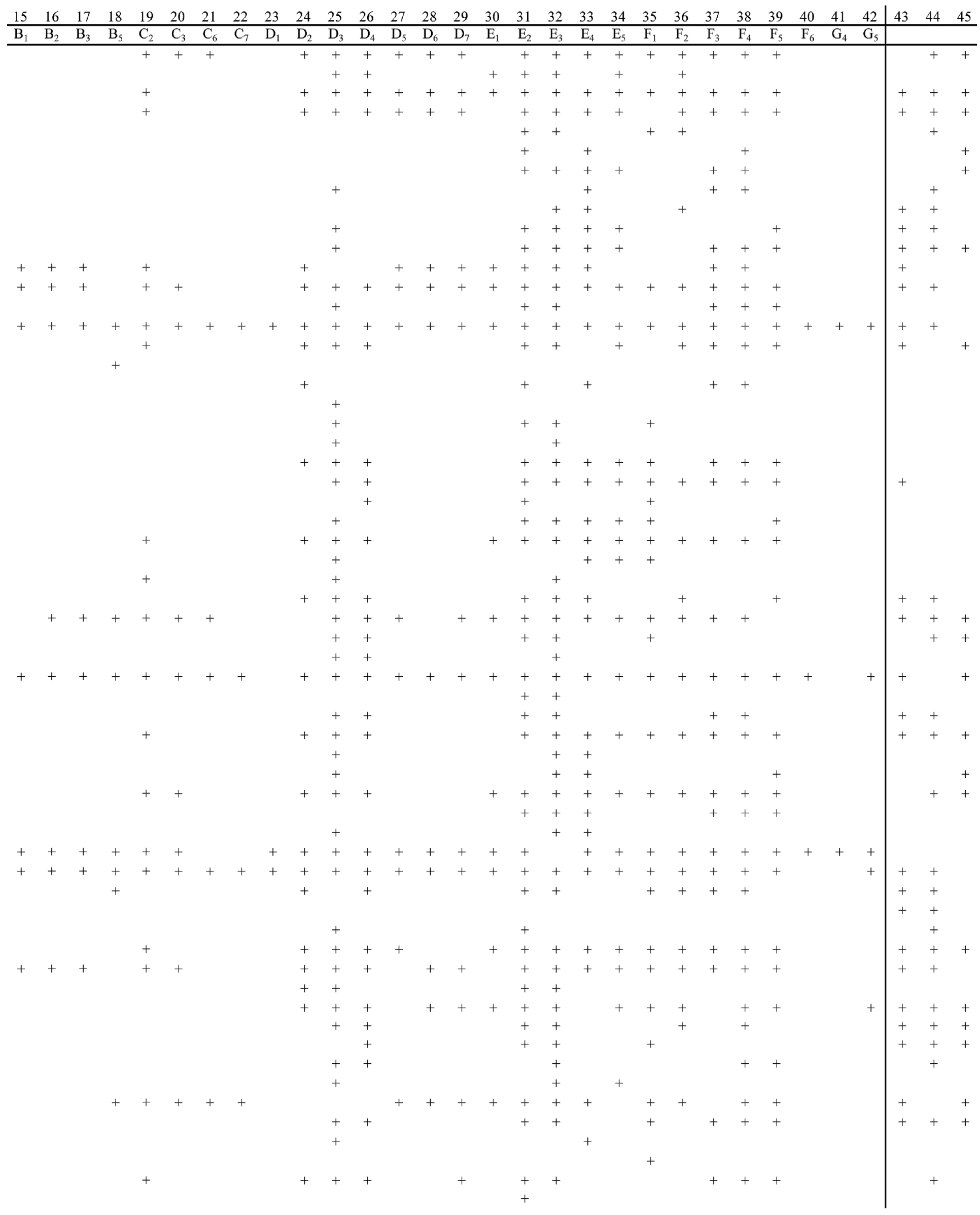




\begin{tabular}{|c|c|c|c|c|c|c|c|c|c|c|c|c|}
\hline 1 & 2 & 3 & 4 & 5 & 6 & 78 & 9 & 10 & 11 & 12 & 13 & 14 \\
\hline 60 & Asperugo procumbens $\mathrm{L}$. & A & SR & $\mathrm{T}$ & $\mathrm{rr}$ & + & & & & & & \\
\hline 61 & Asplenium septentrionale (L.) Hoffm. & $\mathrm{N}$ & $\mathrm{Ch}$ & $\mathrm{H}$ & $\mathrm{f}$ & + & & & & + & + & + \\
\hline 62 & Asplenium trichomanes L. ssp. trichomanes & $\mathrm{N}$ & $\mathrm{Ch}$ & $\mathrm{H}$ & $\mathrm{s}$ & + & & & & + & & \\
\hline 63 & Asplenium viride Huds. & $\mathrm{N}$ & $\mathrm{Ch}$ & $\mathrm{H}$ & $\mathrm{r}$ & + & & & & & & \\
\hline 64 & Aster xsalignus Willd. & EA & $\mathrm{C} / \mathrm{SR}$ & $\mathrm{H}$ & $\mathrm{rr}$ & + & & & & & & \\
\hline 65 & Aster tripolium L. [Tripolium pannonicum (Jacq.) Dobrocz.] & $\mathrm{N}$ & $\mathrm{H}$ & $\mathrm{H}$ & $\mathrm{c}$ & + & & + & + & + & + & + \\
\hline 66 & Athyrium filix-femina (L.) Roth & $\mathrm{N}$ & DF & $\mathrm{H}$ & $\mathrm{s}$ & + & & & & + & + & \\
\hline 67 & Atriplex longipes Drejer ssp. praecox (Hülph.) Turesson & $\mathrm{N}$ & H & $\mathrm{T}$ & $\mathrm{f}$ & + & & + & + & & + & + \\
\hline 68 & Atriplex patula L. & A & SR & $\mathrm{T}$ & $\mathrm{s}$ & + & & & & & & \\
\hline 69 & Atriplex prostrata Boucher ex DC. & $\mathrm{N}$ & $\mathrm{H}$ & $\mathrm{T}$ & $\mathrm{f}$ & + & & & + & & + & \\
\hline 70 & Avenula pubescens (Huds.) Dumort. & A & M & $\mathrm{H}$ & $\mathrm{s}$ & + & & & & & & \\
\hline 71 & Barbarea stricta Andrz. & $\mathrm{N}$ & NTH & $\mathrm{H}$ & $\mathrm{r}$ & + & & & & & & \\
\hline 72 & Barbarea vulgaris $\mathrm{R} . \mathrm{Br}$. & EA & NTH & $\mathrm{H}$ & $\mathrm{s}$ & + & & & + & & & \\
\hline 73 & $\begin{array}{l}\text { Batrachium baudotii (Godr.) Bosch [Ranunculus peltatus Schrank ssp. baudotii (Godr.) C. D. } \\
\text { K. Cook] }\end{array}$ & $\mathrm{N}$ & $\mathrm{W}$ & Hy & $\mathrm{c}$ & + & & + & + & + & + & + \\
\hline 74 & Berteroa incana (L.) DC. & EA & SR & $\mathrm{H}$ & $\mathrm{rr}$ & + & & & & & & \\
\hline 75 & Betula pendula Roth var. pendula & $\mathrm{N}$ & $\mathrm{F}$ & MF & $\mathrm{c}$ & + & & & + & + & + & + \\
\hline 76 & Betula pubescens Ehrh. ssp.pubescens & $\mathrm{N}$ & $\mathrm{F}$ & MF & $\mathrm{s}$ & + & & & & + & + & \\
\hline 77 & Bidens tripartita $\mathrm{L}$. & A & LTH & $\mathrm{T}$ & $\mathrm{s}$ & + & & & & & & \\
\hline 78 & Briza media L. & $\mathrm{N}$ & M & $\mathrm{H}$ & $\mathrm{s}$ & + & & & & & & \\
\hline 79 & Bromus hordeaceus $\mathrm{L}$. & A & $\mathrm{x}$ & $\mathrm{T}$ & $\mathrm{r}$ & + & & & & & & \\
\hline 80 & Calamagrostis $\times$ strigosa (Wahlenb.) Hartm. & $\mathrm{N}$ & LTH & $\mathrm{H}$ & $\mathrm{r}$ & + & & & & & & \\
\hline 81 & Calamagrostis arundinacea (L.) Roth & $\mathrm{N}$ & $\mathrm{F}$ & $\mathrm{H}$ & $\mathrm{s}$ & & & & & & & \\
\hline 82 & Calamagrostis canescens (F. H. Wigg.) Roth & $\mathrm{N}$ & DF & $\mathrm{H}$ & $\mathrm{r}$ & & & & & + & & \\
\hline 83 & Calamagrostis epigejos (L.) Roth & $\mathrm{N}$ & NTH & $\mathrm{G}$ & $\mathrm{f}$ & + & & & & + & + & \\
\hline 84 & Calamagrostis stricta (Timm) Koeler [C. neglecta (Ehrh.) P. Gaertn. et al.] & $\mathrm{N}$ & LTH & $\mathrm{H}$ & $\mathrm{r}$ & + & & & & & + & \\
\hline 85 & Callitriche palustris L. $[$ C. verna L. $]$ & $\mathrm{N}$ & W & $\mathrm{Hy}$ & $\mathrm{rr}$ & + & & & & & & \\
\hline 86 & Calluna vulgaris (L.) Hull & $\mathrm{N}$ & $\mathrm{CF}$ & $\mathrm{Ch}$ & $\mathrm{c}$ & + & & & + & + & + & + \\
\hline 87 & Caltha palustris L. ssp.palustris & $\mathrm{N}$ & M & $\mathrm{H}$ & $\mathrm{s}$ & + & & & & & & \\
\hline 88 & Calystegia sepium (L.) R. Br. ssp. sepium [Convolvulus sepium L.] & $\mathrm{N}$ & NTH & $\mathrm{H}$ & $\mathrm{r}$ & + & & & & & & \\
\hline 89 & Campanula glomerata $\mathrm{L}$. & A & $\mathrm{C} / \mathrm{SR}$ & $\mathrm{H}$ & $\mathrm{rr}$ & + & & & & & & \\
\hline 90 & Campanula patula $\mathrm{L}$. & A & $\mathrm{M}$ & $\mathrm{H}$ & $\mathrm{r}$ & + & & & & & & \\
\hline 91 & Campanula persicifiolia $\mathrm{L}$. & $\mathrm{N}$ & $\mathrm{x}$ & $\mathrm{H}$ & $\mathrm{s}$ & + & & & & & & \\
\hline 92 & Campanula rapunculoides $\mathrm{L}$. & A & $\mathrm{C} / \mathrm{SR}$ & $\mathrm{H}$ & $\mathrm{r}$ & + & & & & & & \\
\hline 93 & Campanula rotundifolia L. ssp. rotundifolia & A & $\mathrm{M}$ & $\mathrm{H}$ & $\mathrm{f}$ & + & & & & + & + & + \\
\hline 94 & Capsella bursa-pastoris (L.) Medik. & A & SR & $\mathrm{T}$ & $\mathrm{s}$ & + & & & & & & \\
\hline 95 & Cardamine hirsuta $\mathrm{L}$. & $\mathrm{N}$ & NTH & $\mathrm{H}$ & $\mathrm{rr}$ & + & & & & & & \\
\hline 96 & Cardamine pratensis L. ssp. pratensis & $\mathrm{N}$ & $\mathrm{M}$ & $\mathrm{H}$ & $\mathrm{s}$ & + & NT & & & + & & \\
\hline 97 & Carduus crispus $\mathrm{L}$. & A & $\mathrm{NTH}$ & $\mathrm{H}$ & $\mathrm{rr}$ & + & & & & & & \\
\hline 98 & Carex brunnescens (Pres.) Poir. & $\mathrm{N}$ & $P$ & $\mathrm{H}$ & $\mathrm{r}$ & + & & & & + & + & \\
\hline 99 & Carex canescens L. & $\mathrm{N}$ & $\mathrm{P}$ & $\mathrm{H}$ & $\mathrm{f}$ & + & & & + & + & + & + \\
\hline 100 & Carex cespitosa $\mathrm{L}$. & $\mathrm{N}$ & M & $\mathrm{H}$ & $\mathrm{r}$ & + & & & & & & \\
\hline 101 & Carex digitata $\mathrm{L}$. & $\mathrm{N}$ & $\mathrm{DF}$ & $\mathrm{H}$ & $\mathrm{f}$ & + & & & & + & + & \\
\hline 102 & Carex echinata Murray [C. stellulata Good.] & $\mathrm{N}$ & $\mathrm{P}$ & $\mathrm{H}$ & $\mathrm{r}$ & + & & & & & & \\
\hline 103 & Carex elata All. & $\mathrm{N}$ & $\mathrm{R}$ & $\mathrm{H}$ & $\mathrm{r}$ & + & & & & & & \\
\hline 104 & Carex hirta L. & A & SR & $\mathrm{G}$ & $\mathrm{r}$ & + & & & & & & \\
\hline 105 & Carex leporina L. [C. ovalis Good.] & A & M & $\mathrm{H}$ & $\mathrm{s}$ & + & & & & + & + & \\
\hline 106 & Carex nigra (L.) Reichard s.1. [C. fusca All.] & $\mathrm{N}$ & $\mathrm{P}$ & $\mathrm{H}$ & $\mathrm{c}$ & + & & & + & + & + & + \\
\hline 107 & Carex pallescens $\mathrm{L}$. & $\mathrm{N}$ & DF & $\mathrm{H}$ & $\mathrm{s}$ & + & & & & + & & \\
\hline 108 & Carex panicea $\mathrm{L}$. & $\mathrm{N}$ & $\mathrm{M}$ & $\mathrm{H}$ & $\mathrm{s}$ & + & & & & + & & \\
\hline 109 & Carex pilulifera $\mathrm{L}$. & $\mathrm{N}$ & M & $\mathrm{H}$ & $\mathrm{f}$ & + & & & & + & + & \\
\hline 110 & Carex rostrata Stokes & $\mathrm{N}$ & $\mathrm{R}$ & Hy & $\mathrm{rr}$ & + & & & & & & \\
\hline 111 & Carex spicata Huds. [C. contigua Hoppe] & A & M & $\mathrm{H}$ & $\mathrm{s}$ & + & & & & & & \\
\hline 112 & Carex vesicaria $\mathrm{L}$. & $\mathrm{N}$ & $\mathrm{R}$ & Hy & $\mathrm{r}$ & + & & & & & + & \\
\hline 113 & Carex viridula Michx. s.1. [C. oederi Retz.; C. serotina Merát] & $\mathrm{N}$ & $\mathrm{P}$ & $\mathrm{H}$ & $\mathrm{r}$ & + & & & & & & \\
\hline 114 & Carum carvi $\mathrm{L}$. & A & M & $\mathrm{H}$ & $\mathrm{r}$ & + & & & & & & \\
\hline 115 & Centaurea cyanus $\mathrm{L}$. & A & SS & $\mathrm{T}$ & $\mathrm{rr}$ & + & & & & & & \\
\hline 116 & Centaurea jacea $\mathrm{L}$. & A & M & $\mathrm{H}$ & $\mathrm{f}$ & + & & & & & + & \\
\hline 117 & Centaurium litorale (Turner) Gilmour & $\mathrm{N}$ & $\mathrm{H}$ & $\mathrm{T}$ & $\mathrm{f}$ & + & & + & + & & + & \\
\hline 118 & Centaurium pulchellum (Sw.) Druce & $\mathrm{N}$ & $\mathrm{H}$ & $\mathrm{T}$ & $\mathrm{r}$ & + & & & & & & \\
\hline 119 & Cerastium arvense $\mathrm{L}$. & $\mathrm{EA}$ & $\mathrm{x}$ & $\mathrm{C}$ & $\mathrm{r}$ & + & & & & & & \\
\hline
\end{tabular}


\begin{tabular}{lllllllllllllllllllllllllllllll}
15 & 16 & 17 & 18 & 19 & 20 & 21 & 22 & 23 & 24 & 25 & 26 & 27 & 28 & 29 & 30 & 31 & 32 & 33 & 34 & 35 & 36 & 37 & 38 & 39 & 40 & 41 & 42 & 43 & 44 & 45 \\
\hline
\end{tabular} $+\quad+\quad++++$

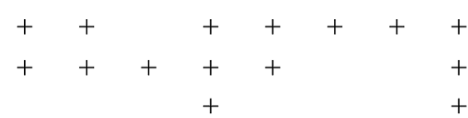

+
+
+

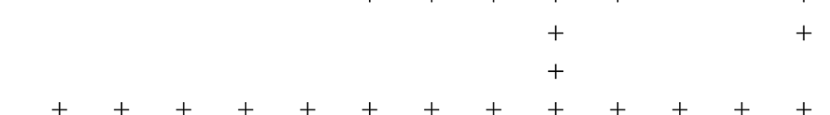

$+\quad+\quad+\quad+\quad+\quad+$

$++\quad++$
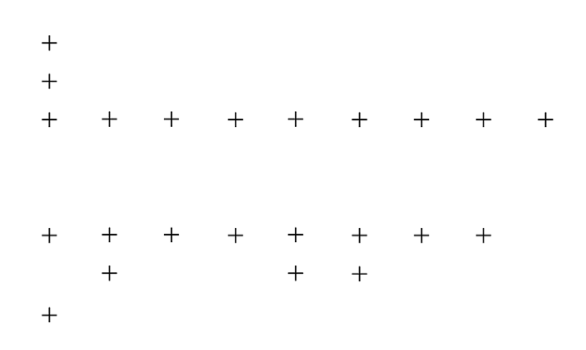

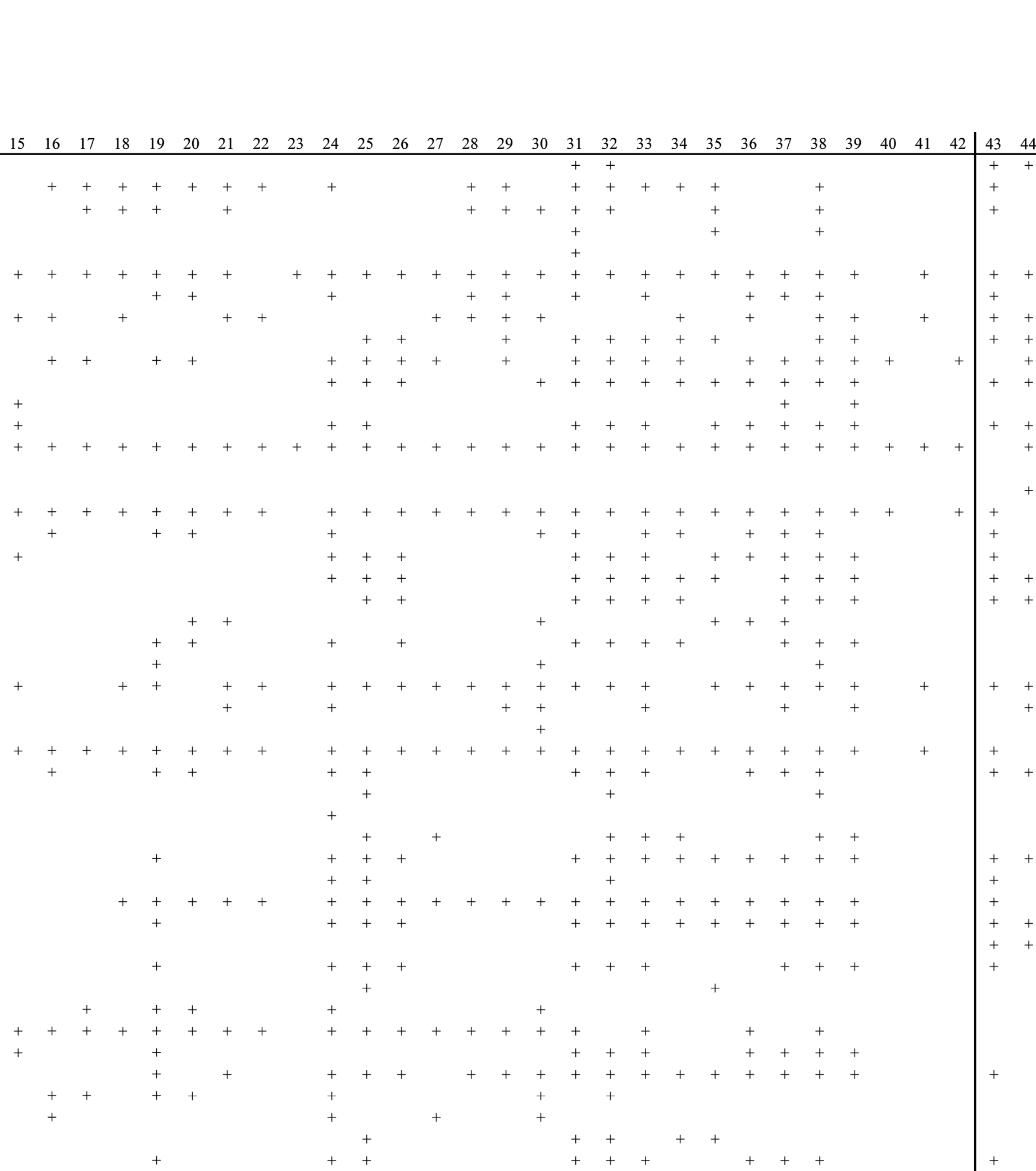

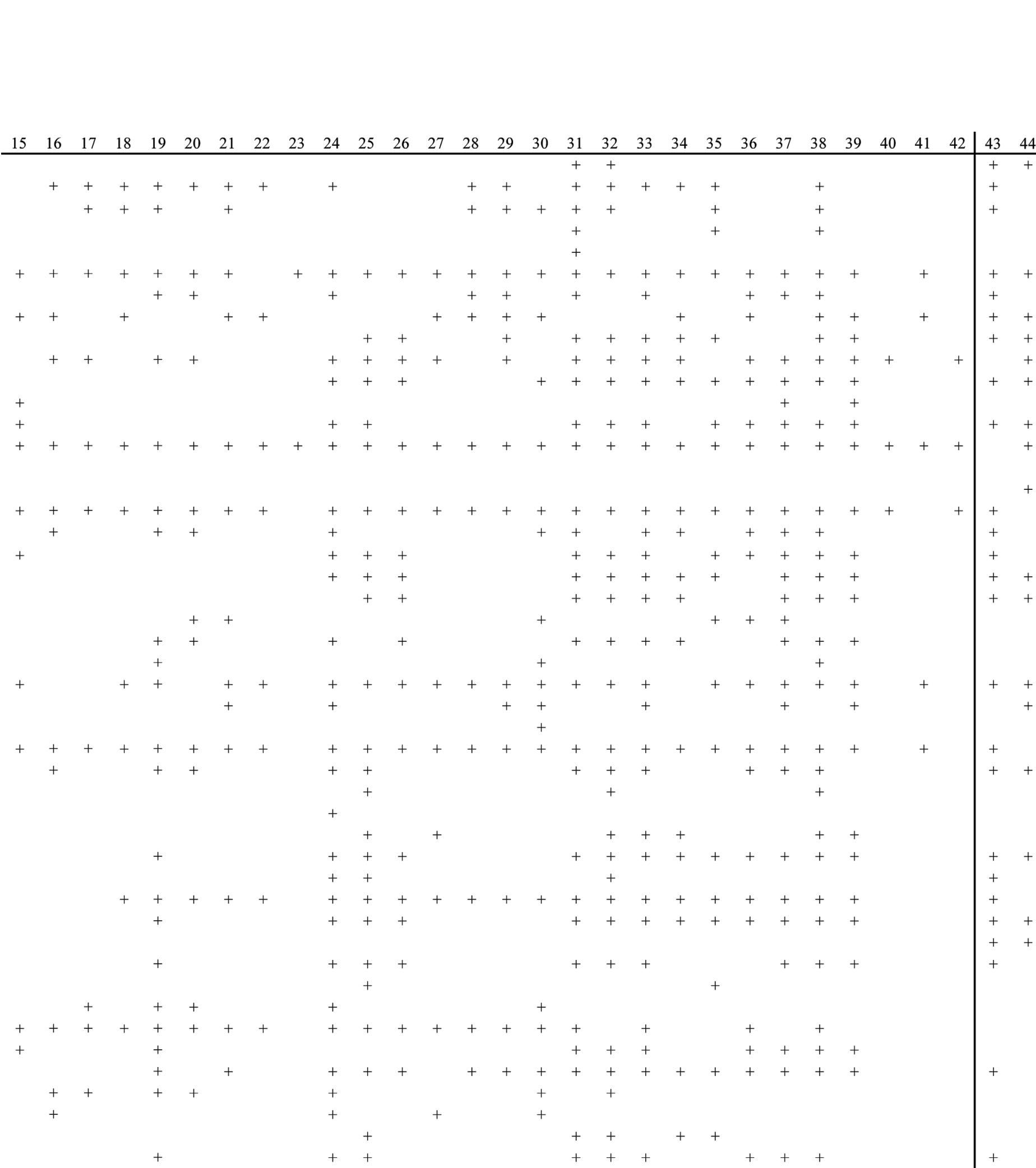
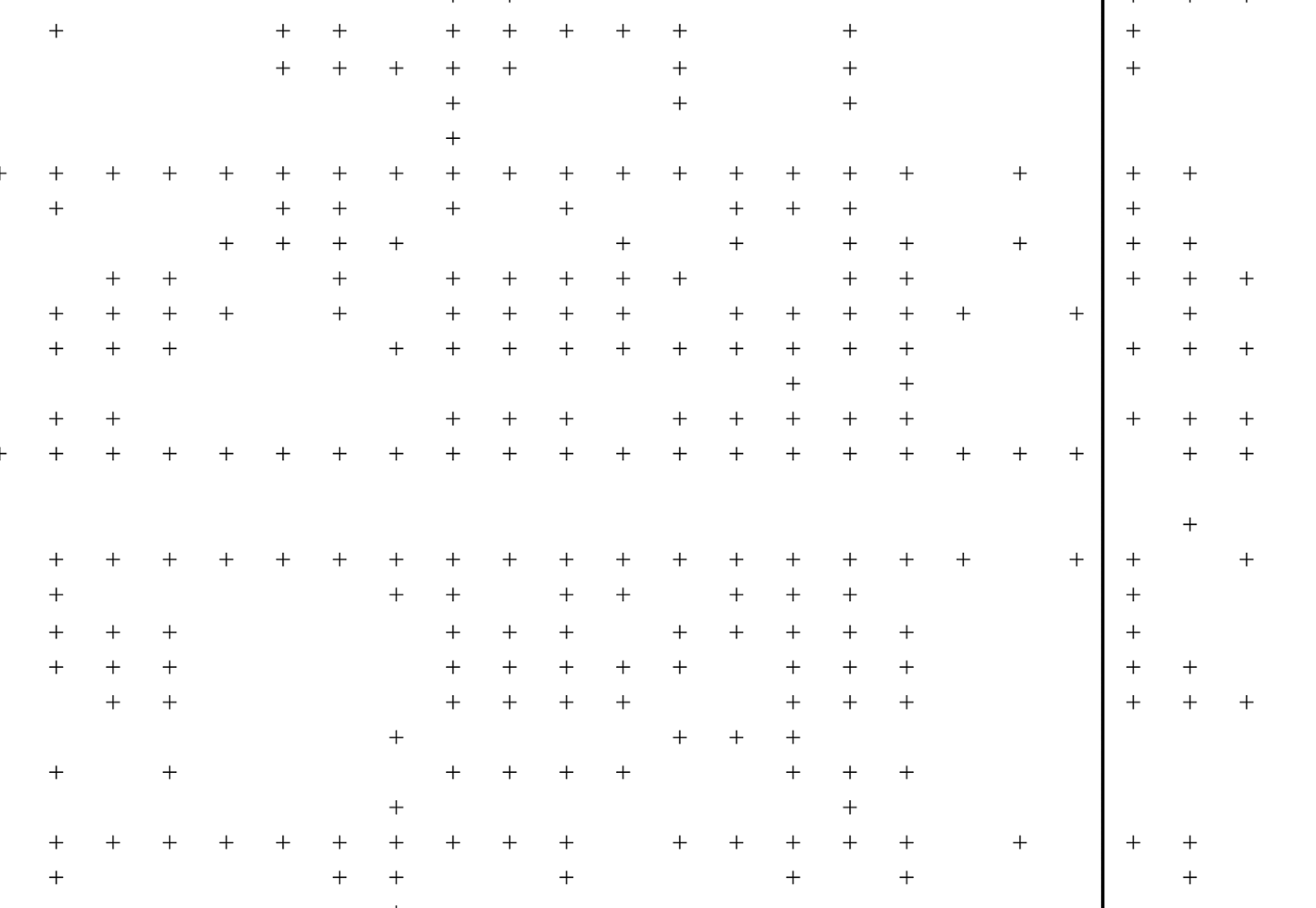

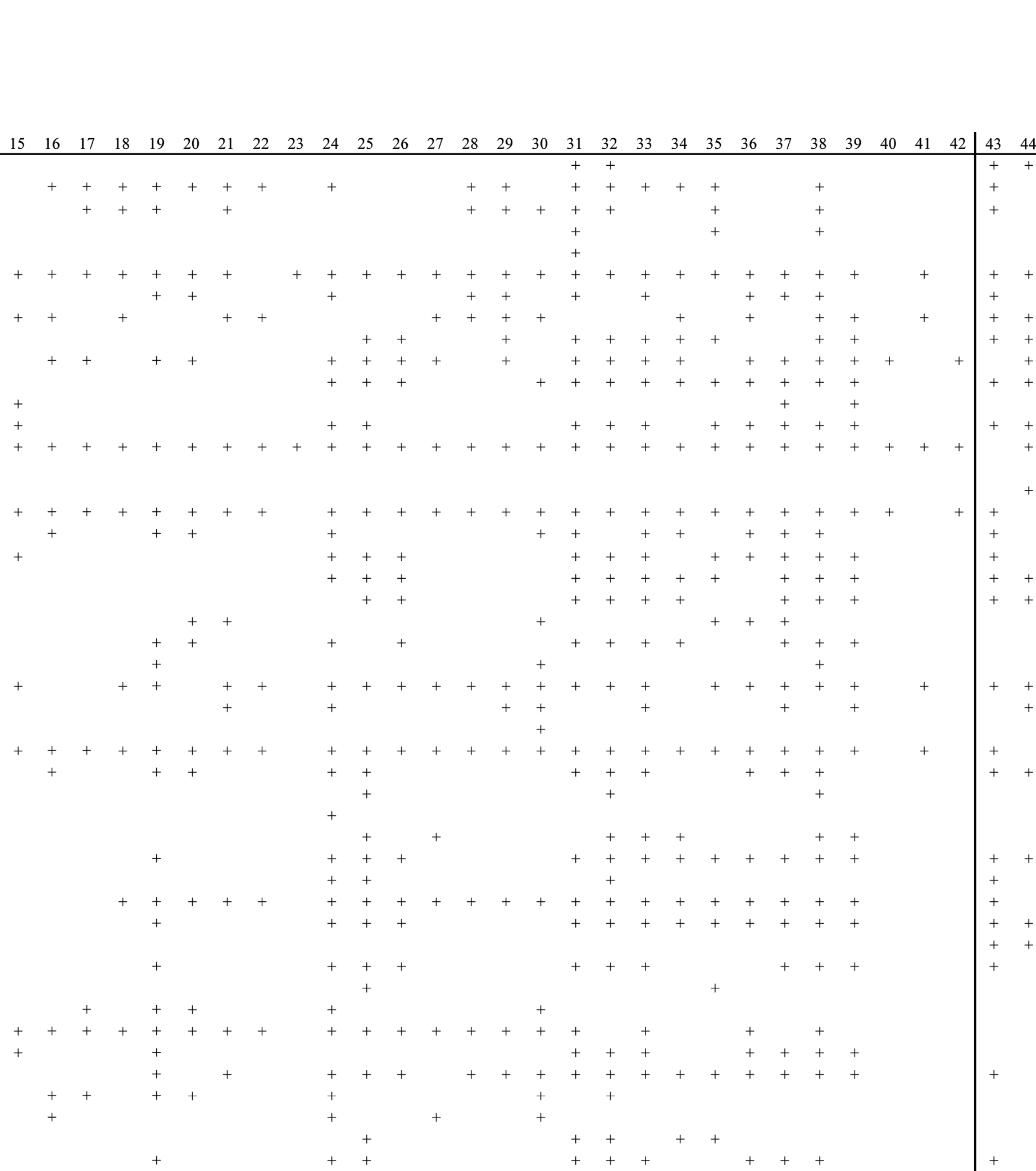

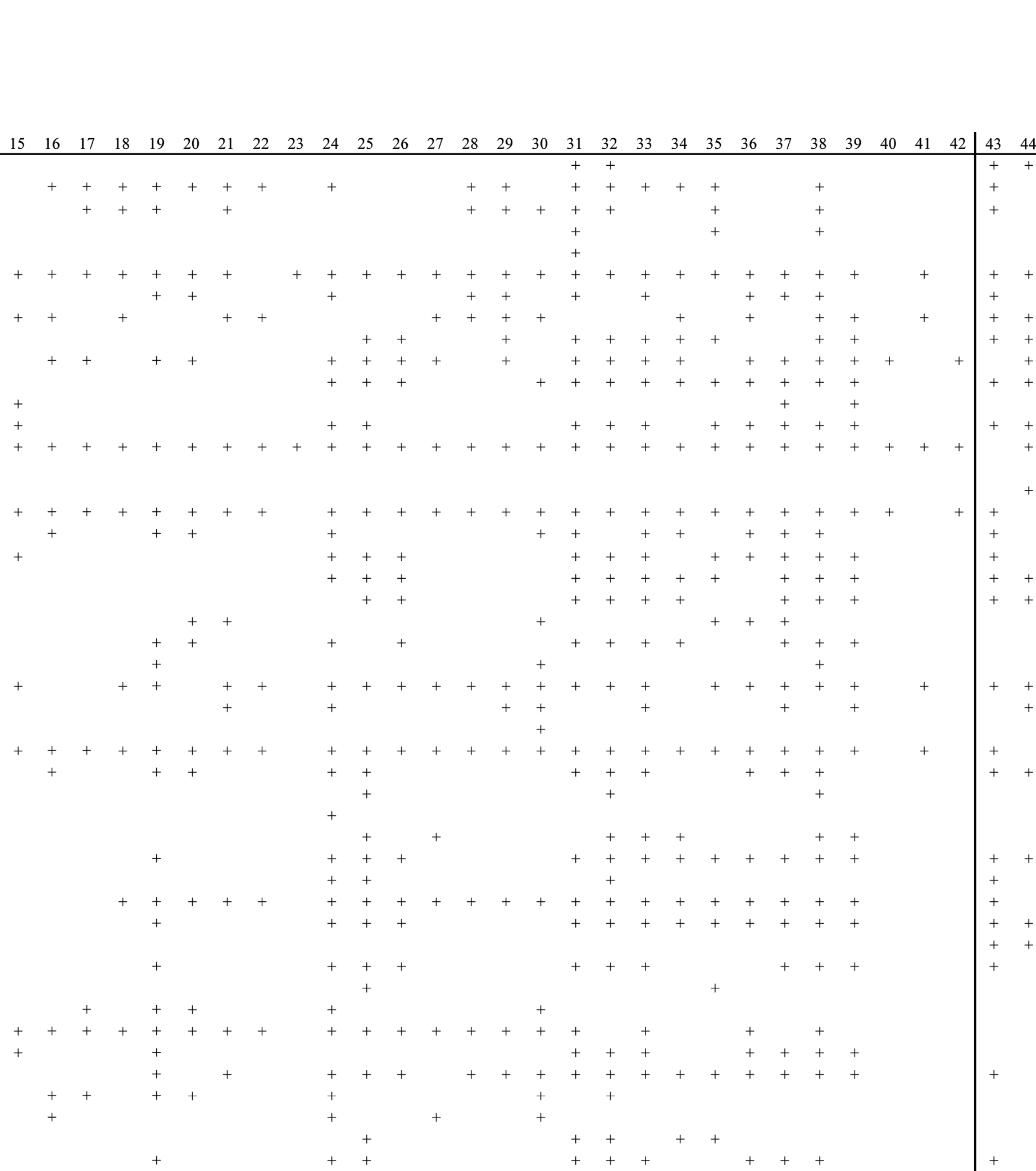

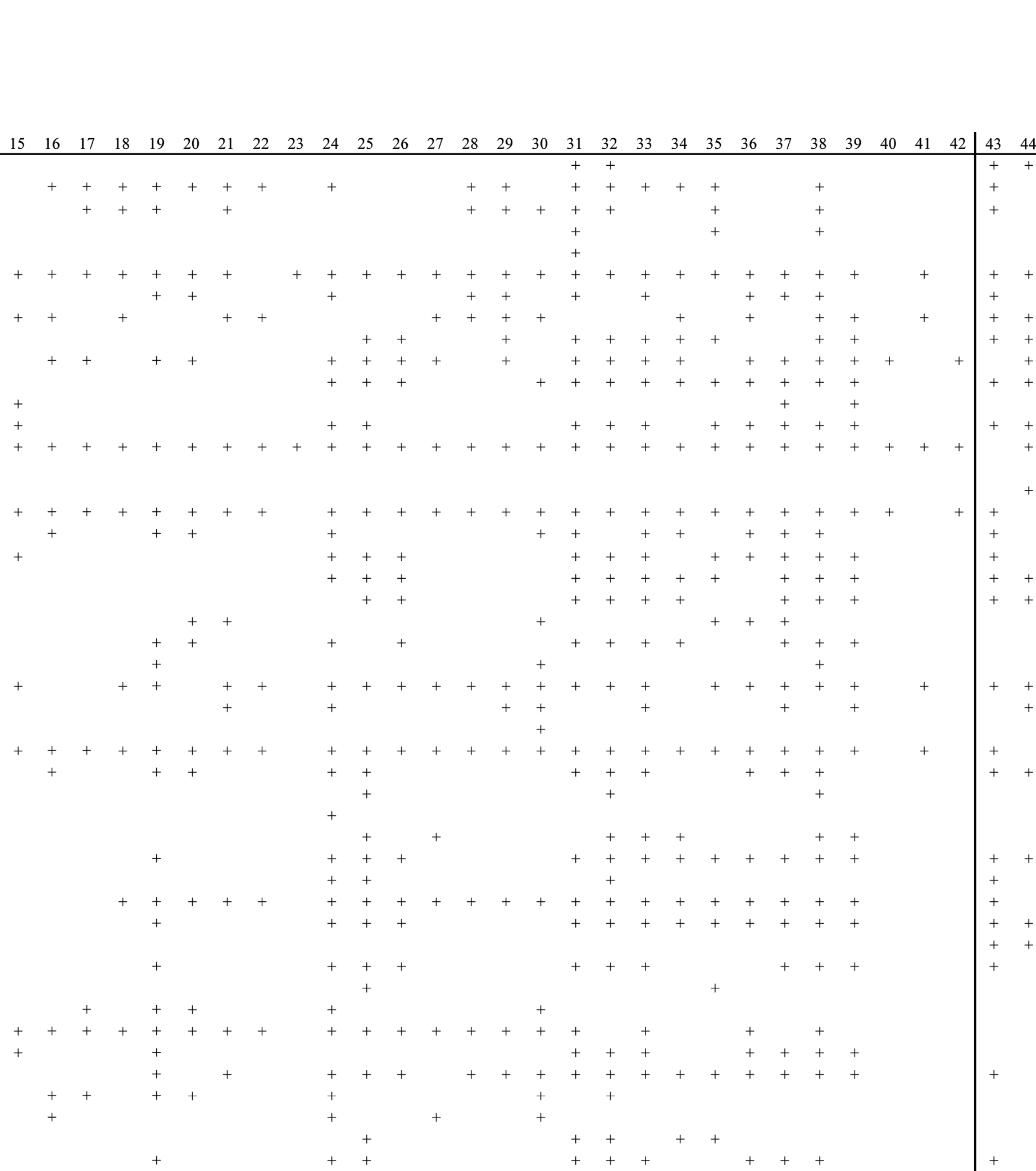

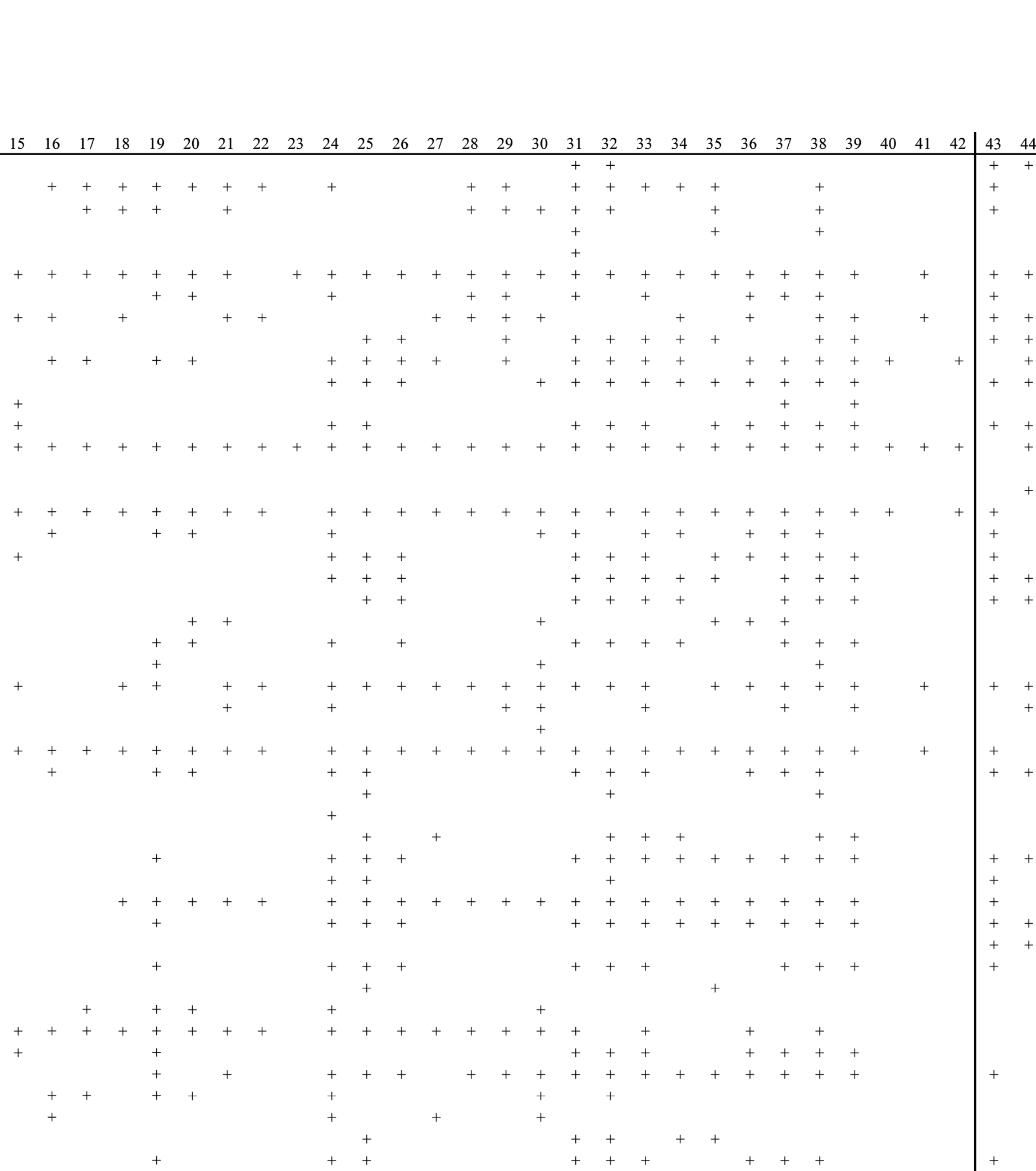

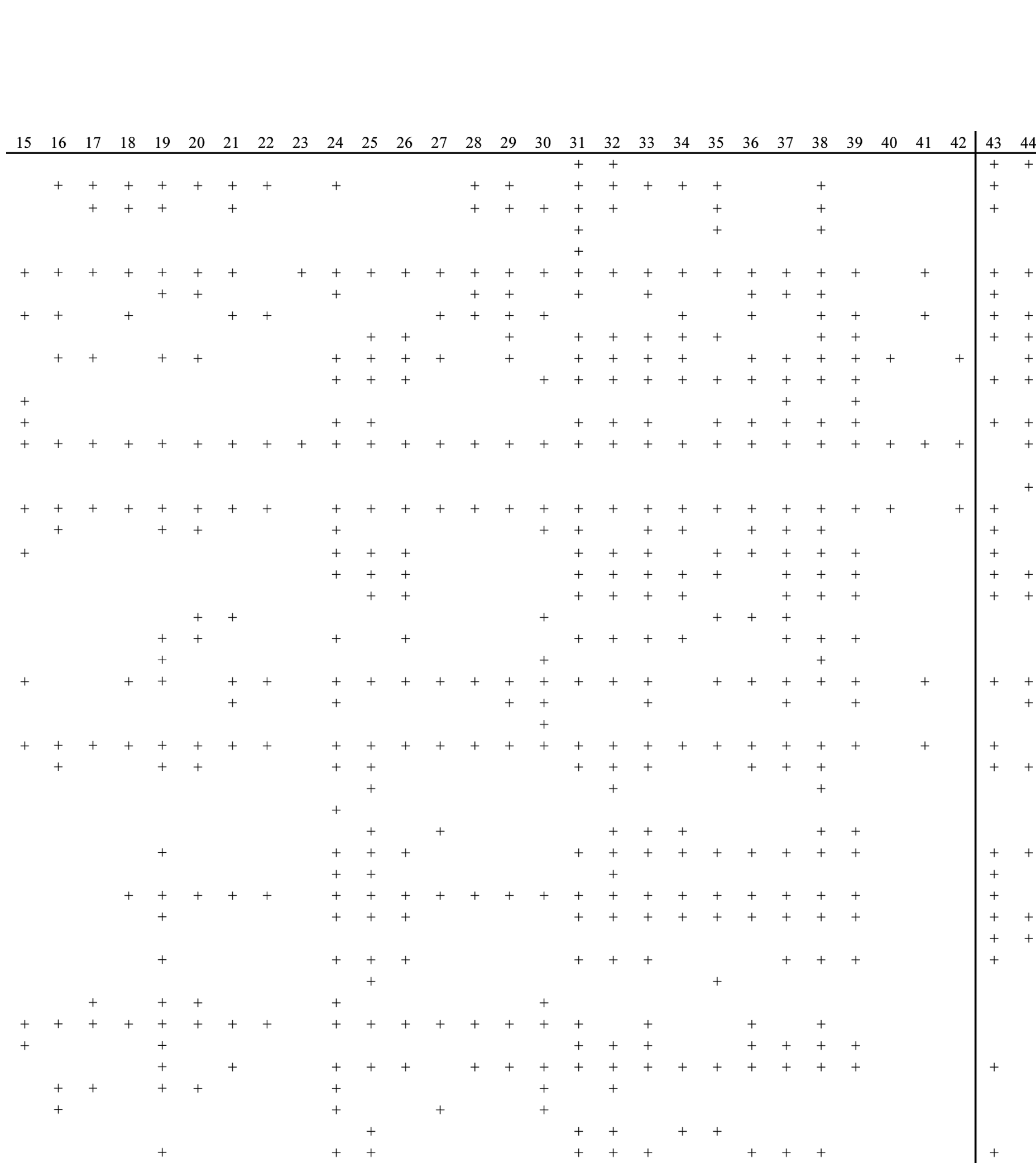

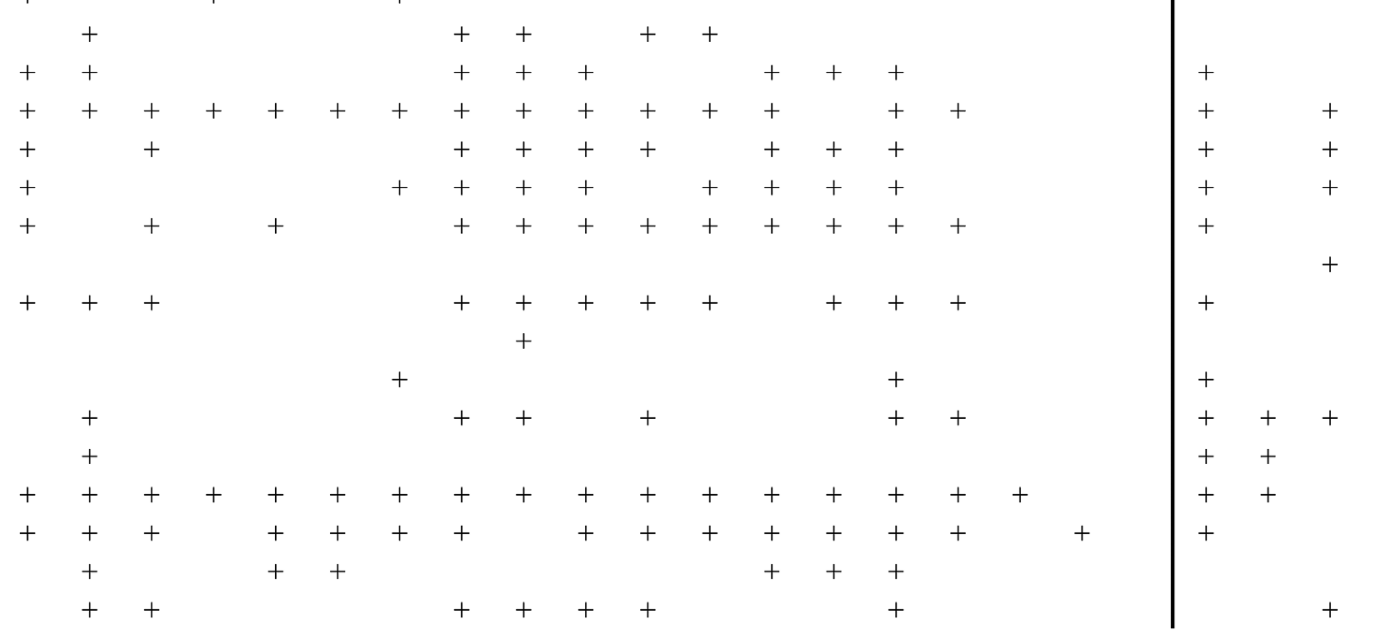




\begin{tabular}{|c|c|c|c|c|c|c|c|c|c|c|c|}
\hline 1 & 2 & 3 & 4 & 5 & 6 & 78 & 9 & $10 \quad 11$ & 12 & 13 & 14 \\
\hline 120 & Cerastium glomeratum Thuill. & $\mathrm{CA}$ & SR & $\mathrm{T}$ & $\mathrm{rr}$ & + & & & & & \\
\hline 121 & Cerastium holosteoides Fr.em. Hyl. [C.vulgatum L.; C. fontanum Baumg. ssp. vulgare] & A & M & $\mathrm{H}$ & $\mathrm{s}$ & + & & & & & \\
\hline 122 & Cerastium semidecandrum L. & $\mathrm{N}$ & $\mathrm{x}$ & $\mathrm{T}$ & $\mathrm{r}$ & + & & & & & \\
\hline 123 & Chelidonium majus $\mathrm{L}$. & A & NTH & $\mathrm{H}$ & $\mathrm{s}$ & + & & & & & \\
\hline 124 & Chenopodium album $\mathrm{L}$. & A & SS & $\mathrm{T}$ & s & & & & & & \\
\hline 125 & Chenopodium glaucum L. [Oxybasis glauca (L.) S. Fuentes et al.] & A & SR & $\mathrm{T}$ & $\mathrm{rr}$ & + & & & & & \\
\hline 126 & Chenopodium polyspermum L. [Lipandra polysperma (L.) S. Fuentes et al.] & A & SS & $\mathrm{T}$ & $\mathrm{rr}$ & + & & & & & \\
\hline 127 & Chenopodium strictum Roth ssp. strictum & $\mathrm{CA}$ & SR & $\mathrm{T}$ & $\mathrm{r}$ & + & & & & & \\
\hline 128 & Chimaphila umbellata (L.) W. P. C. Barton & $\mathrm{N}$ & CF & $\mathrm{Ch}$ & $\mathrm{rr}$ & + & NT & & & & \\
\hline 129 & Chrysosplenium alternifolium $\mathrm{L}$. & $\mathrm{N}$ & DF & $\mathrm{H}$ & $\mathrm{r}$ & + & & & & & \\
\hline 130 & Circaea alpina $\mathrm{L}$. & $\mathrm{N}$ & $\mathrm{CF}$ & $\mathrm{G}$ & $\mathrm{r}$ & + & & & & & \\
\hline 131 & Cirsim arvense (L.) Scop. & $\mathrm{N}$ & NTH & $\mathrm{G}$ & $\mathrm{c}$ & + & & + & + & + & \\
\hline 132 & Cirsium oleraceum (L.) Scop. & EA & SR & $\mathrm{H}$ & $\mathrm{rr}$ & + & vU & & & & \\
\hline 133 & Cirsium palustre (L.) Scop. & $\mathrm{N}$ & M & $\mathrm{H}$ & $\mathrm{r}$ & + & & & & & \\
\hline 134 & Cirsium vulgare (Savi) Ten. & A & SR & $\mathrm{H}$ & $\mathrm{s}$ & + & & & & + & \\
\hline 135 & Clinopodium vulgare L. [Satureja vulgaris (L.) Fritsch] & $\mathrm{N}$ & $\mathrm{X}$ & $\mathrm{H}$ & $\mathrm{r}$ & + & & & & & \\
\hline 136 & Comarum palustre L. [Potentilla palustris (L.) Scop.] & $\mathrm{N}$ & $P$ & $\mathrm{C}$ & $\mathrm{s}$ & + & & & + & + & \\
\hline 137 & Convallaria majalis $\mathrm{L}$. & $\mathrm{N}$ & $\mathrm{DF}$ & $\mathrm{G}$ & $\mathrm{f}$ & + & & & + & + & \\
\hline 138 & Convolvulus arvensis $\mathrm{L}$. & EA & SR & $\mathrm{H}$ & $\mathrm{rr}$ & + & & & & & \\
\hline 139 & Corallorhiza trifida Châtel. & $\mathrm{N}$ & $\mathrm{F}$ & $\mathrm{G}$ & $\mathrm{rr}$ & & & & & & \\
\hline 140 & Corydalis solida (L.) Clairv. & $\mathrm{N}$ & $\mathrm{DF}$ & $\mathrm{G}$ & $\mathrm{r}$ & + & & & & & \\
\hline 141 & Corylus avellana $\mathrm{L}$. & $\mathrm{N}$ & $\mathrm{DF}$ & $\mathrm{NF}$ & $\mathrm{s}$ & + & & & & & \\
\hline 142 & Cotoneaster lucidus Schltdl. & EA & $\mathrm{C} / \mathrm{SR}$ & NF & $\mathrm{rr}$ & + & & & & & \\
\hline 143 & Cotoneaster scandinavicus B. Hylmö [C. integerrimus auct.] & $\mathrm{N}$ & $\mathrm{x}$ & NF & $\mathrm{s}$ & + & & & & & \\
\hline 144 & Crataegus grayana Eggl. [C. flabellata (Bosc ex Spach) K. Koch) p.p.] & EA & SR & $\mathrm{NF}$ & $\mathrm{r}$ & + & & & & & \\
\hline 145 & Crepis tectorum L. ssp. tectorum & $\mathrm{N}$ & $\mathrm{x}$ & $\mathrm{T}$ & $\mathrm{s}$ & + & & & & & \\
\hline 146 & Cuscuta europaea L. ssp. europaea & A & SR & $\mathrm{T}$ & $\mathrm{rr}$ & + & & & & & \\
\hline 147 & Cystopteris fragilis (L.) Bernh. ssp. fragilis & $\mathrm{N}$ & $\mathrm{Ch}$ & $\mathrm{H}$ & $\mathrm{s}$ & + & & & & & \\
\hline 148 & Dactylis glomerata $\mathrm{L}$. & A & M & $\mathrm{H}$ & $\mathrm{s}$ & + & & & & + & \\
\hline 149 & Dactylorhiza maculata (L.) Soó & $\mathrm{N}$ & $\mathrm{P}$ & $\mathrm{G}$ & $\mathrm{r}$ & + & & & + & & \\
\hline 150 & Danthonia decumbens (L.) DC. & $\mathrm{N}$ & M & $\mathrm{H}$ & $\mathrm{s}$ & + & & & + & + & \\
\hline 151 & Dentaria bulbifera $\mathrm{L}$. & $\mathrm{N}$ & DF & $\mathrm{G}$ & $\mathrm{r}$ & + & & & & & \\
\hline 152 & Deschampsia cespitosa (L.) P.Beauv. & $\mathrm{N}$ & M & $\mathrm{H}$ & $\mathrm{f}$ & + & & & + & + & \\
\hline 153 & Deschampsia flexuosa (L.) Trin. [Avenella flexuosa (L.) Drejer] & $\mathrm{N}$ & $\mathrm{CF}$ & $\mathrm{H}$ & $\mathrm{c}$ & + & & + & + & + & + \\
\hline 154 & Descurainia sophia (L.) Webb ex Prantl & A & SR & $\mathrm{T}$ & $\mathrm{r}$ & + & & & & & \\
\hline 155 & Dianthus deltoides L. & A & $\mathrm{x}$ & $\mathrm{H}$ & $\mathrm{s}$ & + & NT & & & & \\
\hline 156 & Diphasiastrum complanatum (L.) Holub ssp. complanatum & $\mathrm{N}$ & $\mathrm{CF}$ & $\mathrm{C}$ & $\mathrm{rr}$ & & & & & & \\
\hline 157 & Dryopteris affinis (Lowe) Fraser-Jenk. & $\mathrm{N}$ & $\mathrm{CF}$ & $\mathrm{H}$ & $\mathrm{r}$ & + & & & & & \\
\hline 158 & Dryopteris carthusiana (Vill.) H. P. Fuchs & $\mathrm{N}$ & $\mathrm{CF}$ & $\mathrm{H}$ & $\mathrm{c}$ & + & & & + & + & + \\
\hline 159 & Dryopteris dilatata (Hoffm.) A. Gray & $\mathrm{N}$ & $\mathrm{CF}$ & $\mathrm{H}$ & $\mathrm{r}$ & + & & & & + & \\
\hline 160 & Dryopteris expansa (C. Presl) Fraser-Jenk. et Jermy & $\mathrm{N}$ & $\mathrm{CF}$ & $\mathrm{H}$ & $\mathrm{s}$ & + & & & + & + & \\
\hline 161 & Dryopteris filix-mas (L.) Schott & $\mathrm{N}$ & $\mathrm{DF}$ & $\mathrm{H}$ & $\mathrm{f}$ & + & & & + & + & \\
\hline 162 & Eleocharis acicularis (L.) Roem. et Schult. & $\mathrm{N}$ & $\mathrm{H}$ & Hy & $\mathrm{r}$ & + & & + & & & \\
\hline 163 & Eleocharis palustris (L.) Roem. et Schult. s.1. & $\mathrm{N}$ & $\mathrm{R}$ & Hy & $\mathrm{r}$ & + & & & & & \\
\hline 164 & Eleocharis parvula (Roem. et Schult.) Link ex Bluf, Nees et Schauer & $\mathrm{N}$ & $\mathrm{H}$ & Hy & $\mathrm{rr}$ & + & & & & & \\
\hline 165 & Eleocharis uniglumis (Link) Schult. & $\mathrm{N}$ & $\mathrm{H}$ & $\mathrm{H}$ & $\mathrm{s}$ & + & & + & & + & \\
\hline 166 & Elymus caninus (L.) L. [Agropyron caninum (L.) P. Beauv.] & $\mathrm{N}$ & $\mathrm{DF}$ & $\mathrm{H}$ & $\mathrm{s}$ & + & & & & & \\
\hline 167 & $\begin{array}{l}\text { Elymus repens (L.) Gould s.1. [Agropyron repens (L.) P. Beauv., Elytrigia repens (L.) Desv. ex } \\
\text { Nevski)] }\end{array}$ & $\mathrm{N}$ & NTH & $\mathrm{G}$ & $\mathrm{f}$ & + & & + & & + & \\
\hline 168 & Empetrum nigrum L. s.l. [incl. E. hermaphroditum Hagerup] & $\mathrm{N}$ & $\mathrm{CF}$ & $\mathrm{C}$ & $\mathrm{c}$ & + & & + & + & + & + \\
\hline 169 & Epilobium adenocaulon Hausskn. s.l. & EA & NTH & $\mathrm{H}$ & $\mathrm{s}$ & + & & & & & \\
\hline 170 & Epilobium angustifolium L. [Chamaenerion angustifolium (L.) Scop.] & $\mathrm{N}$ & NTH & $\mathrm{H}$ & $\mathrm{c}$ & + & & + & + & + & \\
\hline 171 & Epilobium collinum C. C. Gmel. & $\mathrm{N}$ & $\mathrm{Ch}$ & $\mathrm{H}$ & $\mathrm{s}$ & + & & & + & & \\
\hline 172 & Epilobium lamyi F. W. Schultz & $\mathrm{N}$ & SS & $\mathrm{H}$ & $\mathrm{rr}$ & + & $\mathrm{EN}$ & & & & \\
\hline 173 & Epilobium montanum $\mathrm{L}$. & $\mathrm{N}$ & NTH & $\mathrm{H}$ & $\mathrm{s}$ & + & & & & & \\
\hline 174 & Epilobium obscurum Schreb. & $\mathrm{N}$ & M & $\mathrm{H}$ & $\mathrm{r}$ & + & $\mathrm{EN}$ & & & & \\
\hline 175 & Epilobium palustre L. var. palustre & $\mathrm{N}$ & $P$ & $\mathrm{H}$ & $\mathrm{r}$ & + & & & & & \\
\hline 176 & Epipactis helleborine (L.) Crantz & $\mathrm{N}$ & $\mathrm{DF}$ & $\mathrm{G}$ & $\mathrm{r}$ & + & & & & & \\
\hline 177 & Equisetum arvense L. ssp. arvense & $\mathrm{N}$ & NTH & $\mathrm{G}$ & $\mathrm{f}$ & + & & & + & + & \\
\hline 178 & Equisetum fluviatile L. & $\mathrm{N}$ & $\mathrm{R}$ & Hy & $\mathrm{r}$ & + & & & & & \\
\hline 179 & Equisetum palustre L. & $\mathrm{N}$ & M & $\mathrm{G}$ & $\mathrm{r}$ & & & & & & \\
\hline
\end{tabular}



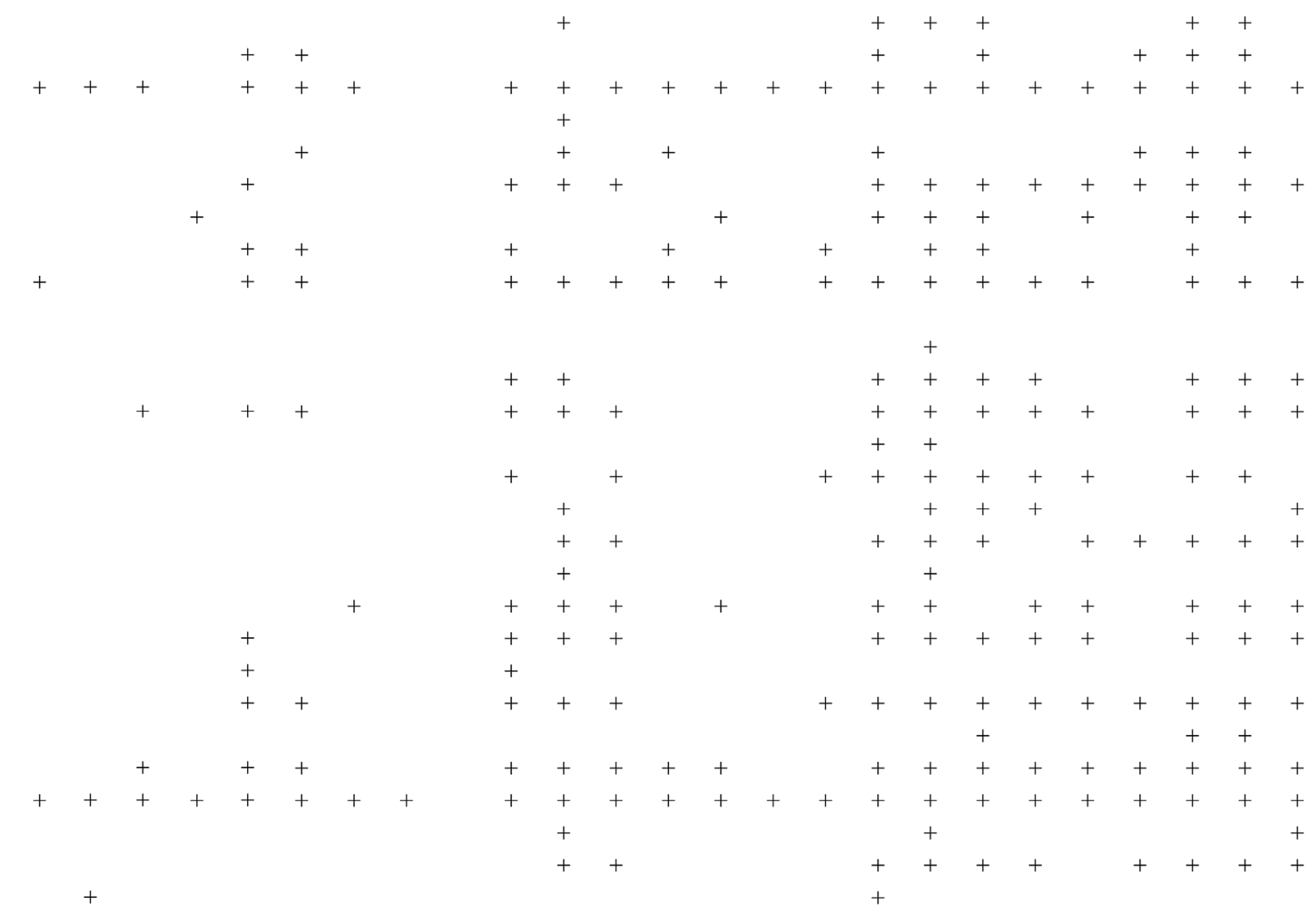

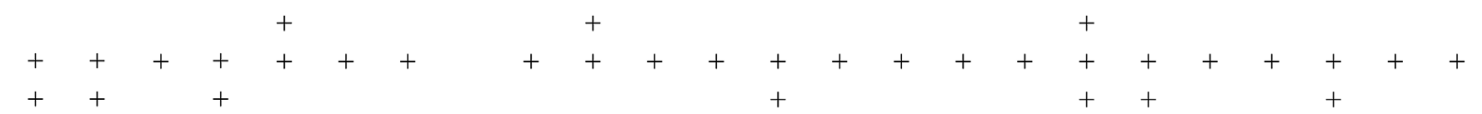
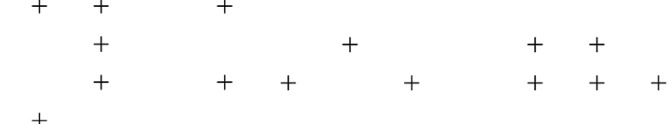

$\begin{array}{lllllll} & + & & & & \\ + & & & & & \\ + & + & & + & & \\ + & + & + & + & + & \\ + & + & & + & + & +\end{array}$

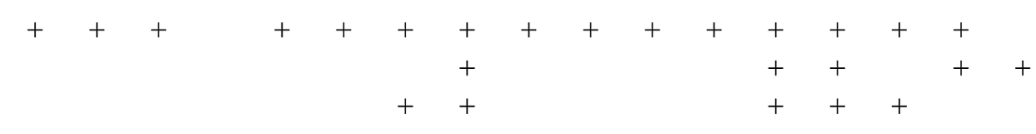

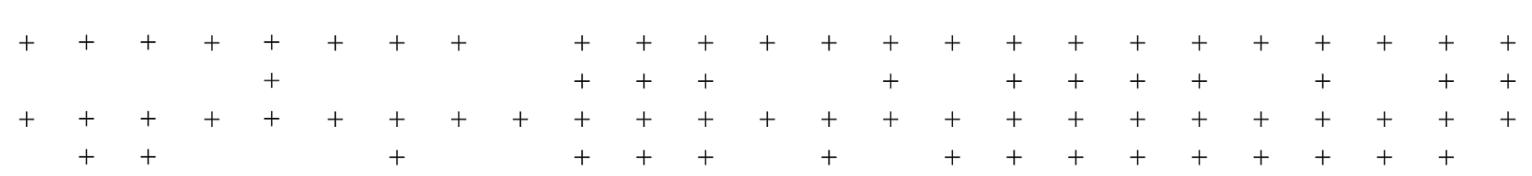

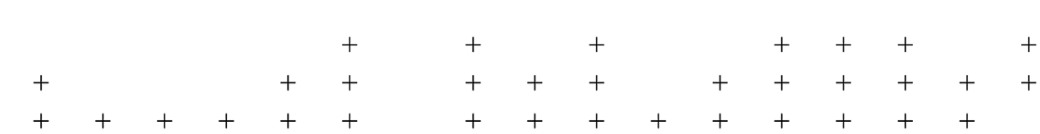

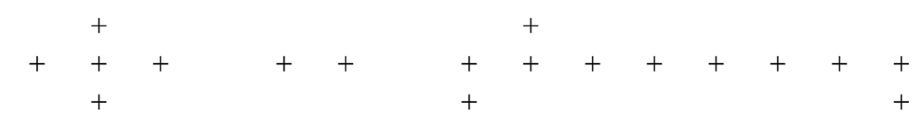

\begin{tabular}{l|lll}
+ & + & + & + \\
+ & + & + & \\
+ & + & + \\
& + & + & + \\
& & + &
\end{tabular}




\begin{tabular}{|c|c|c|c|c|c|c|c|c|c|c|c|c|}
\hline 1 & 2 & 3 & 4 & 5 & 67 & 78 & 9 & 10 & 11 & 12 & 13 & 14 \\
\hline 180 & Equisetum pratense Ehrh. & $\mathrm{N}$ & $\mathrm{DF}$ & $\mathrm{G}$ & $\mathrm{r}+$ & + & & & & & & \\
\hline 181 & Equisetum sylvaticum $\mathrm{L}$. & $\mathrm{N}$ & DF & G & $s+$ & + & & & & & & \\
\hline 182 & Erigeron acer L. ssp. acer & A & $\mathrm{x}$ & $\mathrm{H}$ & $r+$ & + & & & & & & \\
\hline 183 & Eriophorum angustifolium Honck. & $\mathrm{N}$ & $P$ & Hy & $s+$ & + & & & + & & & \\
\hline 184 & Eriophorum vaginatum $\mathrm{L}$ & $\mathrm{N}$ & $\mathrm{P}$ & $\mathrm{H}$ & $r+$ & + & & & & & & \\
\hline 185 & Erophila verna (L.) Chevall. [Draba verna $\mathrm{L}]$. & A & $\mathrm{x}$ & $\mathrm{T}$ & $\mathrm{r}+$ & + & & & & & & \\
\hline 186 & Erysimum cheiranthoides L. ssp. cheiranthoides & A & SS & $\mathrm{T}$ & $r+$ & + & & & & & & \\
\hline 187 & Eupatorium cannabinum $\mathrm{L}$. & $\mathrm{N}$ & LTH & $\mathrm{H}$ & $\mathrm{r}$ & + & & & & & & \\
\hline 188 & Euphorbia cyparissias $\mathrm{L}$. & EA & $\mathrm{X}$ & G & $\mathrm{r}$ & & & & & & & \\
\hline 189 & Euphorbia helioscopia L. & A & SS & $\mathrm{T}$ & $r+$ & + & & & & & & \\
\hline 190 & Euphrasia nemorosa (Pers.) Wallr. & A & M & $\mathrm{T}$ & $r+$ & + & & & & & & \\
\hline 191 & Euphrasia stricta J. F. Lehm. & A & M & $\mathrm{T}$ & $s+$ & + & & & + & & & \\
\hline 192 & Fallopia convolvulus (L.) A. Löve & A & SS & $\mathrm{T}$ & $s+$ & + & & & & & & \\
\hline 193 & Fallopia dumetorum (L.) Holub & $\mathrm{N}$ & NTH & $\mathrm{T}$ & $f+$ & + & & & & + & + & \\
\hline 194 & Festuca arundinacea Schreb. [F. elatior L., Schedonorus arundinaceus (Schreb.) Dumort.] & $\mathrm{N}$ & M & $\mathrm{H}$ & $\mathrm{r}+$ & + & & & & & & \\
\hline 195 & Festuca brevipila Tracey $[F$. trachyphylla (Hack.) Krajina] & EA & $\mathrm{X}$ & $\mathrm{H}$ & $\mathrm{rr}$ & + & & & & & & \\
\hline 196 & Festuca ovina $\mathrm{L}$. & $\mathrm{N}$ & $\mathrm{x}$ & $\mathrm{H}$ & $c+$ & + & & & & + & + & + \\
\hline 197 & Festuca pratensis Huds. [Schedonorus pratensis (Huds.) P. Beauv.] & A & M & $\mathrm{H}$ & $r+$ & + & & & & & & \\
\hline 198 & Festuca rubra L. ssp. rubra & $\mathrm{N}$ & M & $\mathrm{H}$ & $s+$ & + & & & & & + & \\
\hline 199 & Festuca salina Natho et Stohr [F. rubra L. ssp. (var., fo.) litoralis auct.] & $\mathrm{N}$ & $\mathrm{H}$ & $\mathrm{H}$ & $\mathrm{c}$ & + & & + & + & + & + & + \\
\hline 200 & Ficaria verna Huds. [Ranunculus ficaria L.] & $\mathrm{N}$ & $\mathrm{DF}$ & G & $\mathrm{r}+$ & + & & & & & & \\
\hline 201 & Filago arvensis $\mathrm{L}$. & A & $\mathrm{x}$ & $\mathrm{T}$ & $r+$ & + & & & & & & \\
\hline 202 & Filipendula ulmaria (L.) Maxim. & $\mathrm{N}$ & M & $\mathrm{H}$ & $c+$ & + & & & + & + & + & + \\
\hline 203 & Filipendula vulgaris Moench & A & $\mathrm{x}$ & $\mathrm{H}$ & $r+$ & + & & & & & & \\
\hline 204 & Fragaria moschata Weston [F. muricata Mill.] & EA & $\mathrm{x}$ & $\mathrm{H}$ & $\mathrm{rr}+$ & + & & & & & & \\
\hline 205 & Fragaria vesca $\mathrm{L}$. & $\mathrm{N}$ & $\mathrm{x}$ & $\mathrm{H}$ & $f+$ & + & & & + & + & + & + \\
\hline 206 & Fraxinus excelsior $\mathrm{L}$. & $\mathrm{N}$ & $\mathrm{DF}$ & MF & $s+$ & + & & & & & + & \\
\hline 207 & Fumaria officinalis $\mathrm{L}$. & A & SS & $\mathrm{T}$ & $r+$ & + & & & & & & \\
\hline 208 & Gagea minima (L.) Ker Gawl. & A & $\mathrm{DF}$ & G & $s+$ & + & & & & & & \\
\hline 209 & Galeopsis bifida Boenn. & $\mathrm{N}$ & LTH & $\mathrm{T}$ & $r+$ & + & & & & & & \\
\hline 210 & Galeopsis speciosa Mill. & A & NTH & $\mathrm{T}$ & $r+$ & + & & & & & & \\
\hline 211 & Galeopsis tetrahit $\mathrm{L}$. & A & NTH & $\mathrm{T}$ & $\mathrm{f}$ & & & & & & + & + \\
\hline 212 & Galium album Mill. [G. mollugo auct. p.p.] & EA & $\mathrm{x}$ & $\mathrm{H}$ & $r+$ & + & & & & & & \\
\hline 213 & Galium aparine L. & $\mathrm{N}$ & NTH & $\mathrm{T}$ & $s+$ & + & & & & & & \\
\hline 214 & Galium boreale L. & $\mathrm{N}$ & M & $\mathrm{H}$ & $s+$ & + & & & & & + & \\
\hline 215 & Galium elongatum C. Presl [G.palustre L. ssp. elongatum (C. Presl) Lange] & $\mathrm{N}$ & $\mathrm{R}$ & $\mathrm{H}$ & $\mathrm{r}$ & + & & & & & & \\
\hline 216 & Galium palustre L. ssp. palustre & $\mathrm{N}$ & $\mathrm{R}$ & $\mathrm{H}$ & $c+$ & + & & + & + & + & + & + \\
\hline 217 & Galium spurium L. s.1. & A & SS & $\mathrm{T}$ & $\mathrm{r}+$ & + & & & & & & \\
\hline 218 & Galium uliginosum $\mathrm{L}$. & $\mathrm{N}$ & M & $\mathrm{H}$ & $r+$ & + & & & & + & + & \\
\hline 219 & Galium verum $\mathrm{L}$. & $\mathrm{N}$ & $\mathrm{X}$ & $\mathrm{H}$ & $s+$ & + & $\mathrm{VU}$ & & & & + & \\
\hline 220 & Galium $\times$ pomeranicum Retz. & EA & M & $\mathrm{H}$ & $\mathrm{rr}$ & + & & & & & & \\
\hline 221 & Geranium pusillum L. & A & SS & $\mathrm{T}$ & $\mathrm{r}+$ & + & & & & & & \\
\hline 222 & Geranium robertianum $\mathrm{L}$. & $\mathrm{N}$ & NTH & $\mathrm{H}$ & $f+$ & + & & & & & & \\
\hline 223 & Geranium sylvaticum L. & $\mathrm{N}$ & NTH & $\mathrm{H}$ & $s+$ & + & & & & & & \\
\hline 224 & Geum rivale $\mathrm{L}$. & $\mathrm{N}$ & M & $\mathrm{H}$ & $\mathbf{s}+$ & + & & & & & & \\
\hline 225 & Geum urbanum $\mathrm{L}$. & $\mathrm{N}$ & $\mathrm{NTH}$ & $\mathrm{H}$ & $f+$ & + & & & & & & \\
\hline 226 & Glaux maritima L. [Lysimachia maritima (L.) Galasso et al.] & $\mathrm{N}$ & $\mathrm{H}$ & $\mathrm{H}$ & $c+$ & + & & + & + & + & + & + \\
\hline 227 & Glyceria fluitans (L.) R. Br. & $\mathrm{N}$ & $\mathrm{R}$ & Hy & $r+$ & + & & & & & & \\
\hline 228 & Gnaphalium sylvaticum L. [Omalotheca sylvatica (L.) Schultz-Bip. et F.W. Schultz] & A & $\mathrm{NTH}$ & $\mathrm{H}$ & $s+$ & + & & & & & & \\
\hline 229 & Gnaphalium uliginosum $\mathrm{L}$. & A & SS & $\mathrm{T}$ & $r+$ & + & & & & & & \\
\hline 230 & Goodyera repens (L.) R.Br. & $\mathrm{N}$ & $\mathrm{CF}$ & $\mathrm{H}$ & $s+$ & + & & & & & + & \\
\hline 231 & Gymnocarpium dryopteris (L.) Newman & $\mathrm{N}$ & $\mathrm{CF}$ & $\mathrm{G}$ & $f+$ & + & & & & + & + & \\
\hline 232 & Hepatica nobilis Schreb. & $\mathrm{N}$ & $\mathrm{DF}$ & $\mathrm{H}$ & $s+$ & + & & & & & & \\
\hline 233 & Heracleum sibiricum L. [Heracleum sphondylium L. ssp. sibiricum (L.) Sink.] & A & NTH & $\mathrm{H}$ & $\mathrm{rr}+$ & + & & & & & & \\
\hline 234 & Hieracium caesium ( $\mathrm{Fr}.) \mathrm{Fr}$. & $\mathrm{N}$ & $\mathrm{x}$ & $\mathrm{H}$ & $\mathrm{r}$ & + & & & & & + & \\
\hline 235 & Hieracium cymosum L. [Pilosella cymosa (L.) F. W. Schultz et Schultz-Bip. p.p.] & $\mathrm{N}$ & $\mathrm{x}$ & $\mathrm{H}$ & $\mathrm{r}$ & & & & & & & \\
\hline 236 & Hieracium laevigatum Willd. s. str. & $\mathrm{N}$ & $\mathrm{F}$ & $\mathrm{H}$ & $\mathrm{r}$ & + & & & & & & \\
\hline 237 & Hieracium linifolium Saelan ex Lindeb. [H. tridentatum Fr.] & $\mathrm{N}$ & $\mathrm{X}$ & $\mathrm{H}$ & $\mathrm{s}$ & + & & & & & & \\
\hline 238 & Hieracium murorum L. s.l. & $\mathrm{N}$ & DF & $\mathrm{H}$ & $\mathrm{s}$ & + & & & & & & \\
\hline 239 & $\begin{array}{l}\text { Hieracium oistophyllum Pugsley [H. fuscocinereum Norrl. em. S. Bräut.; H. sagittatum (Lindeb.) } \\
\text { Norrl.] }\end{array}$ & $\mathrm{N}$ & $\mathrm{F}$ & $\mathrm{H}$ & $\mathrm{rr}$ & + & & & & & & \\
\hline
\end{tabular}




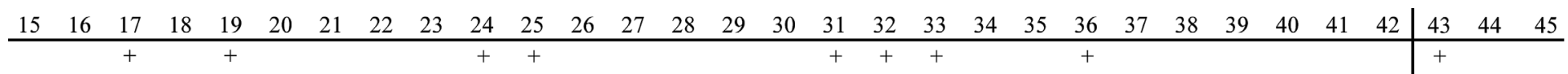

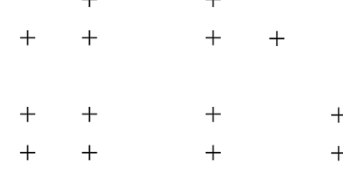

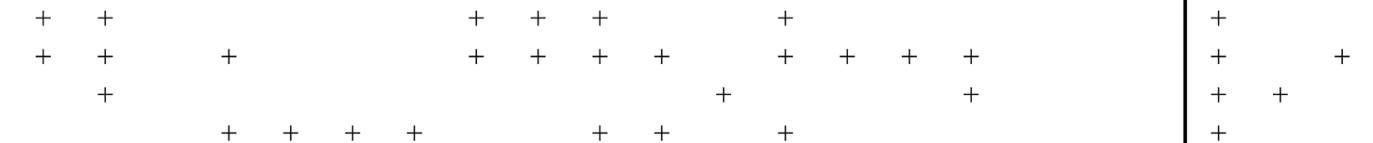




\begin{tabular}{|c|c|c|c|c|c|c|c|c|c|c|c|c|}
\hline 1 & 2 & 3 & 4 & 5 & 6 & 78 & 9 & 10 & 11 & 12 & 13 & 14 \\
\hline 240 & Hieracium pilosella L. [Pilosella officinarum F.W. Schultz et Schultz-Bip.] & $\mathrm{N}$ & $\mathrm{X}$ & $\mathrm{H}$ & $\mathrm{s}$ & + & & & & & & \\
\hline 241 & Hieracium suecicum Fr. [Pilosella $\times$ floribunda (Wimm. et Grab) Arv.-Touv. p.p.] & $\mathrm{N}$ & $\mathrm{X}$ & $\mathrm{H}$ & $\mathrm{rr}$ & & & & & & & \\
\hline 242 & Hieracium umbellatum $\mathrm{L}$. & $\mathrm{N}$ & LTH & $\mathrm{H}$ & $\mathrm{c}$ & & & + & + & + & + & + \\
\hline 243 & Hieracium vulgatum Fr. [H. lachenalii C. C. Gmel.] & $\mathrm{N}$ & $\mathrm{F}$ & $\mathrm{H}$ & $\mathrm{f}$ & + & & & & + & + & \\
\hline 244 & Hippophaë rhamnoides $\mathrm{L}$. & $\mathrm{N}$ & $\mathrm{X}$ & NF & $\mathrm{rr}$ & + & & & & & & \\
\hline 245 & Hippuris vulgaris $\mathrm{L}$. & $\mathrm{N}$ & W & Hy & $\mathrm{rr}$ & + & & & & & & \\
\hline 246 & Holcus mollis $\mathrm{L}$. & EA & M & $\mathrm{H}$ & $\mathrm{r}$ & + & & & & & & \\
\hline 247 & Hordeum vulgare L. & $\mathrm{CA}$ & SR & $\mathrm{T}$ & $\mathrm{rr}$ & + & & & & & & \\
\hline 248 & Huperzia selago (L.) Bernh. ex Schrank et Mart. ssp. selago & $\mathrm{N}$ & $\mathrm{CF}$ & $\mathrm{C}$ & $\mathrm{s}$ & + & & & & + & & + \\
\hline 249 & Hyoscyamus niger L. & A & SR & $\mathrm{T}$ & $\mathrm{rr}$ & + & & & & & & \\
\hline 250 & Hypericum maculatum Crantz & A & $\mathrm{x}$ & $\mathrm{H}$ & $\mathrm{s}$ & + & & & & & & \\
\hline 251 & Hypericum perforatum $\mathrm{L}$. & $\mathrm{N}$ & $\mathrm{X}$ & $\mathrm{H}$ & $\mathrm{s}$ & + & & & & & + & \\
\hline 252 & Hypochoeris maculata $\mathrm{L}$. & $\mathrm{N}$ & $\mathrm{X}$ & $\mathrm{H}$ & $\mathrm{rr}$ & + & & & & & & \\
\hline 253 & Iris pseudacorus $\mathrm{L}$. & $\mathrm{N}$ & $\mathrm{R}$ & Hy & $\mathrm{s}$ & + & & & & + & + & \\
\hline 254 & Isatis tinctoria $\mathrm{L}$. & A & SR & $\mathrm{H}$ & $\mathrm{r}$ & + & & & & & & \\
\hline 255 & Juncus articulatus L. var. articulatus & A & M & $\mathrm{H}$ & $\mathrm{r}$ & + & & & & & & \\
\hline 256 & Juncus bufonius L. ssp. bufonius & A & SS & $\mathrm{T}$ & $\mathrm{s}$ & + & & & & & + & \\
\hline 257 & Juncus bulbosus L. [J. supinus Moench] & $\mathrm{N}$ & $\mathrm{P}$ & $\mathrm{H}$ & $\mathrm{rr}$ & + & & & & & & \\
\hline 258 & Juncus compressus Jacq. & $\mathrm{N}$ & M & $\mathrm{G}$ & $\mathrm{r}$ & + & & & & & & \\
\hline 259 & Juncus conglomeratus L. & A & M & $\mathrm{H}$ & $\mathrm{r}$ & + & & & & & & \\
\hline 260 & Juncus effusus L. & $\mathrm{N}$ & M & $\mathrm{H}$ & $\mathrm{s}$ & + & & & & + & & \\
\hline 261 & Juncus filiformis L. & $\mathrm{N}$ & $P$ & $\mathrm{G}$ & $\mathrm{r}$ & + & & & & + & + & \\
\hline 262 & Juncus gerardii Loisel. & $\mathrm{N}$ & $\mathrm{H}$ & $\mathrm{G}$ & $\mathrm{c}$ & + & & + & + & + & + & + \\
\hline 263 & Juniperus communis L. ssp. communis & $\mathrm{N}$ & $\mathrm{CF}$ & NF & $\mathrm{c}$ & + & & + & & + & + & + \\
\hline 264 & Lamium amplexicaule $\mathrm{L}$. & A & SS & $\mathrm{T}$ & $\mathrm{r}$ & + & & & & & & \\
\hline 265 & Lamium hybridum Vill. [L. incisum Willd.] & A & SS & $\mathrm{T}$ & $\mathrm{r}$ & + & & & & & & \\
\hline 266 & Lamium purpureum $\mathrm{L}$. & A & SS & $\mathrm{T}$ & $\mathrm{r}$ & + & & & & & & \\
\hline 267 & Lapsana communis L. & A & NTH & $\mathrm{H}$ & $\mathrm{s}$ & + & & & & & & \\
\hline 268 & Lathyrus montanus Bernh. [L. linifolius (Reichard) Bässler] & $\mathrm{N}$ & DF & $\mathrm{G}$ & $\mathrm{rr}$ & + & & & & & & \\
\hline 269 & Lathyrus niger (L.) Bernh. & $\mathrm{N}$ & $\mathrm{DF}$ & G & $\mathrm{rr}$ & + & & & & & & \\
\hline 270 & Lathyrus pratensis $\mathrm{L}$. & $\mathrm{N}$ & M & $\mathrm{H}$ & $\mathrm{s}$ & + & & & + & + & + & \\
\hline 271 & Lathyrus vernus (L.) Bernh. & $\mathrm{N}$ & $\mathrm{DF}$ & $\mathrm{G}$ & $\mathrm{r}$ & + & & & & & & \\
\hline 272 & Ledum palustre L. [Rhododendron tomentosum Harmaja] & $\mathrm{N}$ & $\mathrm{CF}$ & $\mathrm{C}$ & $\mathrm{r}$ & + & & & & & & \\
\hline 273 & Lemna trisulca $\mathrm{L}$. & $\mathrm{N}$ & W & Hy & $\mathrm{r}$ & + & & & & & & \\
\hline 274 & Leontodon autumnalis L. [Scorzoneroides autumnalis (L.) Moench] & $\mathrm{N}$ & M & $\mathrm{H}$ & $\mathrm{f}$ & + & & + & + & & + & + \\
\hline 275 & Leonurus cardiaca L. ssp. cardiaca & EA & $\mathrm{SR}$ & $\mathrm{H}$ & $\mathrm{r}$ & + & & & & & & \\
\hline 276 & Leucanthemum vulgare Lam. & A & M & $\mathrm{H}$ & $\mathrm{s}$ & + & & & & & & \\
\hline 277 & Levisticum officinale W. D. J. Koch & EA & $\mathrm{C} / \mathrm{SR}$ & $\mathrm{H}$ & $\mathrm{rr}$ & & & & & & & \\
\hline 278 & Leymus arenarius (L.) Hochst. [Elymus arenarius L.] & $\mathrm{N}$ & LTH & $\mathrm{G}$ & $\mathrm{c}$ & + & & & + & + & + & + \\
\hline 279 & Lilium martagon L. & EA & $\mathrm{DF}$ & $\mathrm{G}$ & $\mathrm{r}$ & + & & & & & & \\
\hline 280 & Linaria vulgaris Mill. & $\mathrm{N}$ & SR & $\mathrm{G}$ & $\mathrm{r}$ & + & & & & & & \\
\hline 281 & Linnaea borealis $\mathrm{L}$. & $\mathrm{N}$ & $\mathrm{CF}$ & $\mathrm{C}$ & f & + & & & & & & \\
\hline 282 & Linum catharticum $\mathrm{L}$. & $\mathrm{N}$ & $\mathrm{x}$ & $\mathrm{T}$ & $\mathrm{r}$ & + & & & & & & \\
\hline 283 & Listera cordata (L.) R.Br. [Neottia cordata (L.) Rich.] & $\mathrm{N}$ & $\mathrm{CF}$ & $\mathrm{G}$ & $\mathrm{r}$ & + & & & & + & + & \\
\hline 284 & Listera ovata (L.) R.Br. [Neottia ovata (L.) Bluff \& Fingerh.] & $\mathrm{N}$ & $\mathrm{DF}$ & $\mathrm{G}$ & $\mathrm{r}$ & + & & & & & & \\
\hline 285 & Lithospermum arvense L. [Buglossoides arvensis (L.) M. I. Johnston] & A & SS & $\mathrm{T}$ & $\mathrm{rr}$ & + & $\mathrm{EN}$ & & & & & \\
\hline 286 & Lolium multiflorum Lam. & EA & M & $\mathrm{H}$ & $\mathrm{rr}$ & & & & & & & \\
\hline 287 & Lolium perenne $\mathrm{L}$. & EA & M & $\mathrm{H}$ & $\mathrm{r}$ & + & & & & & & \\
\hline 288 & Lonicera xylosteum $\mathrm{L}$. & $\mathrm{N}$ & DF & NF & $\mathrm{s}$ & + & & & & & & \\
\hline 289 & Lotus corniculatus L. & $\mathrm{N}$ & M & $\mathrm{H}$ & $\mathrm{s}$ & + & & & & & + & \\
\hline 290 & Lupinus polyphyllus Lindl. & EA & SR & $\mathrm{H}$ & $\mathrm{r}$ & + & & & & & & \\
\hline 291 & Luzula campestris (L.) DC. & A & $\mathrm{x}$ & $\mathrm{H}$ & $\mathrm{s}$ & + & & & & & & \\
\hline 292 & Luzula multiflora (Ret.) Lej. ssp. multiflora & A & M & $\mathrm{H}$ & f & + & & & & + & + & + \\
\hline 293 & Luzula pallescens Sw. [L. pallidula Kirschner] & $\mathrm{N}$ & $\mathrm{F}$ & $\mathrm{H}$ & $\mathrm{r}$ & & & & & & & \\
\hline 294 & Luzula pilosa (L.) Willd. & $\mathrm{N}$ & $\mathrm{F}$ & $\mathrm{H}$ & $\mathrm{c}$ & + & & & & + & + & + \\
\hline 295 & Lycopodium annotinum L. ssp. annotinum [Spinulum annotinum (L.) A. Haines] & $\mathrm{N}$ & $\mathrm{CF}$ & $\mathrm{C}$ & $\mathrm{f}$ & + & & & & + & + & \\
\hline 296 & Lycopodium clavatum L. ssp. clavatum & $\mathrm{N}$ & $\mathrm{CF}$ & $\mathrm{C}$ & $\mathrm{rr}$ & + & & & & & & \\
\hline 297 & Lycopus europaeus L. & $\mathrm{N}$ & DF & $\mathrm{H}$ & $\mathrm{r}$ & + & & & & & & \\
\hline 298 & Lysimachia nummularia $\mathrm{L}$. & EA & M & $\mathrm{C}$ & $\mathrm{rr}$ & + & & & & & & \\
\hline 299 & Lysimachia punctata L. & $\mathrm{CA}$ & M & $\mathrm{H}$ & $\mathrm{rr}$ & + & & & & & & \\
\hline 300 & Lysimachia thyrsiflora $\mathrm{L}$. & $\mathrm{N}$ & $\mathrm{R}$ & $\mathrm{H}$ & $\mathrm{r}$ & + & & & & & & \\
\hline
\end{tabular}




\begin{tabular}{|c|c|c|c|c|c|c|c|c|c|c|c|c|}
\hline 1 & 2 & 3 & 4 & 5 & 6 & 78 & 9 & 10 & 11 & 12 & 13 & 14 \\
\hline 301 & Lysimachia vulgaris L. & $\mathrm{N}$ & M & $\mathrm{H}$ & $\mathrm{c}$ & + & & + & + & + & + & + \\
\hline 302 & Lythrum salicaria $\mathrm{L}$. & $\mathrm{N}$ & M & $\mathrm{H}$ & $\mathrm{c}$ & + & & + & + & + & + & + \\
\hline 303 & Maianthemum bifolium (L.) F. W. Schm. & $\mathrm{N}$ & $\mathrm{F}$ & $\mathrm{G}$ & $\mathrm{f}$ & + & & & & + & + & + \\
\hline 304 & Malus domestica Borkh. & EA & $\mathrm{C} / \mathrm{SR}$ & MF & $\mathrm{r}$ & + & & & & & & \\
\hline 305 & Malva moschata $\mathrm{L}$. & EA & $\mathrm{SR}$ & $\mathrm{H}$ & $\mathrm{rr}$ & + & & & & & & \\
\hline 306 & Malva pusilla $\mathrm{Sm}$. & A & SR & $\mathrm{T}$ & $\mathrm{rr}$ & + & & & & & & \\
\hline 307 & $\begin{array}{l}\text { Matricaria discoidea DC. [Chamomilla suaveolens (Pursh) Rydb., M. matricarioides (Less.) } \\
\text { Porter] }\end{array}$ & EA & SR & $\mathrm{T}$ & $\mathrm{r}$ & + & & & & & & \\
\hline 308 & Matricaria chamomilla L. [Chamomilla recutita (L.) Rauschert] & A & SS & $\mathrm{T}$ & $\mathrm{rr}$ & + & & & & & & \\
\hline 309 & Melampyrum arvense L. & A & M & $\mathrm{T}$ & $\mathrm{r}$ & + & $\mathrm{VU}$ & & & & & \\
\hline 310 & Melampyrum pratense $\mathrm{L}$. & $\mathrm{N}$ & $\mathrm{CF}$ & $\mathrm{T}$ & $\mathrm{c}$ & + & & & & + & + & + \\
\hline 311 & Melampyrum sylvaticum $\mathrm{L}$. & $\mathrm{N}$ & $\mathrm{F}$ & $\mathrm{T}$ & $\mathrm{f}$ & + & & & & & & \\
\hline 312 & Melandrium album (Mill.) Garcke [Silene latifolia Poir. ssp. alba (Mill.) Greuter \& Burdet] & EA & SR & $\mathrm{H}$ & rr & + & & & & & & \\
\hline 313 & Melandrium noctiflorum (L.) Fr. [Silene noctiflora L.] & EA & Ss & $\mathrm{T}$ & $\mathrm{rr}$ & + & & & & & & \\
\hline 314 & Melandrium rubrum (Weigel) Garcke [Silene dioica (L.) Clairv.] & $\mathrm{N}$ & NTH & $\mathrm{H}$ & $\mathrm{s}$ & + & & & & & & \\
\hline 315 & Melica mutans $\mathrm{L}$. & $\mathrm{N}$ & DF & $\mathrm{H}$ & $\mathrm{f}$ & + & & & & + & + & \\
\hline 316 & Mentha $\times$ gracilis Sole & EA & $\mathrm{C} / \mathrm{SR}$ & $\mathrm{H}$ & $\mathrm{rr}$ & + & & & & & & \\
\hline 317 & Mentha arvensis $\mathrm{L}$. & $\mathrm{N}$ & M & $\mathrm{H}$ & $\mathrm{r}$ & + & & & & + & + & \\
\hline 318 & Menyanthes trifoliata $\mathrm{L}$. & $\mathrm{N}$ & $P$ & G & $\mathrm{r}$ & + & & & & & & \\
\hline 319 & Milium effusum $\mathrm{L}$. & $\mathrm{N}$ & DF & $\mathrm{H}$ & $r$ & + & & & & & & \\
\hline 320 & Moehringia trinervia (L.) Clairv. & $\mathrm{N}$ & NTH & $\mathrm{H}$ & $\mathrm{f}$ & + & & & & + & + & \\
\hline 321 & Moneses uniflora (L.) A.Gray & $\mathrm{N}$ & $\mathrm{CF}$ & $\mathrm{C}$ & $\mathrm{r}$ & + & & & & + & & \\
\hline 322 & Monotropa hypopitys L. ssp. hypopitys & $\mathrm{N}$ & $\mathrm{CF}$ & $\mathrm{G}$ & $\mathrm{s}$ & & & & & & + & \\
\hline 323 & Montia fontana $\mathrm{L}$. & $\mathrm{N}$ & W & Hy & $\mathrm{rr}$ & + & & & & & & \\
\hline 324 & Mycelis muralis (L.) Dumort. [Lactuca muralis (L.) Gaertn.] & $\mathrm{N}$ & NTH & $\mathrm{H}$ & $\mathrm{s}$ & + & & & & & & \\
\hline 325 & Myosotis arvensis (L.) Hill. & A & SS & $\mathrm{T}$ & $\mathrm{r}$ & + & & & & & & \\
\hline 326 & Myosotis laxa Lehm. ssp. baltica (Sam.) Hyl. ex Nordh. & $\mathrm{N}$ & LTH & $\mathrm{H}$ & $\mathrm{s}$ & + & & & + & & & \\
\hline & Myosotis laxa Lehm. ssp. caespitosa (Schultz) Hyl. ex Nordh. & $\mathrm{N}$ & M & $\mathrm{H}$ & $\mathrm{r}$ & + & & & & & & \\
\hline 327 & Myosotis ramosissima Rochel [M. collina Hoffm.] & $\mathrm{N}$ & $\mathrm{X}$ & $\mathrm{T}$ & $\mathrm{r}$ & + & & & & & & \\
\hline 328 & Myosotis scorpioides L. [M. palustris (L.) L. em. Rchb.] & $\mathrm{N}$ & M & $\mathrm{H}$ & $\mathrm{r}$ & + & & & & & & \\
\hline 329 & Myosotis stricta Link ex Roem. et Schult. [M. micrantha Pall.] & $\mathrm{N}$ & $\mathrm{X}$ & $\mathrm{T}$ & $\mathrm{r}$ & + & & & & & & \\
\hline 330 & Myosurus minimus $\mathrm{L}$. & A & SS & $\mathrm{T}$ & $\mathrm{rr}$ & + & & & & & & \\
\hline 331 & Myriophyllum spicatum L. s.l. & $\mathrm{N}$ & W & Hy & $\mathrm{s}$ & & & & & & + & \\
\hline 332 & Nardus stricta $\mathrm{L}$. & $\mathrm{N}$ & M & $\mathrm{H}$ & $\mathrm{r}$ & + & NT & & & + & & \\
\hline 333 & Neottia nidus-avis (L.) Rich. & $\mathrm{N}$ & $\mathrm{DF}$ & G & $\mathrm{r}$ & + & & & & & & \\
\hline 334 & Odontites litoralis (Fr.) Fr. ssp. littoralis & $\mathrm{N}$ & $\mathrm{H}$ & $\mathrm{T}$ & $\mathrm{f}$ & + & & + & + & & + & \\
\hline 335 & Odontites vulgaris Moench [O. serotina (Lam.) Rchb. O. rubra Gilib.] & A & M & $\mathrm{T}$ & $\mathrm{r}$ & + & & & & & & \\
\hline 336 & Ophioglossum vulgatum $\mathrm{L}$. & $\mathrm{N}$ & LTH & $\mathrm{G}$ & $\mathrm{s}$ & + & & & + & & + & \\
\hline 337 & Origanum vulgare L. & $\mathrm{N}$ & $\mathrm{X}$ & $\mathrm{C}$ & $\mathrm{r}$ & + & & & & & & \\
\hline 338 & Orthilia secunda (L.) House & $\mathrm{N}$ & $\mathrm{CF}$ & $\mathrm{C}$ & $\mathrm{f}$ & + & & & & + & + & \\
\hline 339 & Oxalis acetosella $\mathrm{L}$. & $\mathrm{N}$ & $\mathrm{F}$ & $\mathrm{G}$ & $\mathrm{s}$ & + & & & & + & + & \\
\hline 340 & Oxycoccus palustris Pers. [Vaccinium oxycoccos L.] & $\mathrm{N}$ & $\mathrm{P}$ & $\mathrm{C}$ & $\mathrm{r}$ & + & & & & & & \\
\hline 341 & Padus avium Mill. [Prunus padus L. ssp. padus ] & $\mathrm{N}$ & DF & MF & $\mathrm{s}$ & + & & & & & & \\
\hline 342 & Papaver somniferum $\mathrm{L}$. & EA & SS & $\mathrm{T}$ & rr & + & & & & & & \\
\hline 343 & Paris quadrifolia L. & $\mathrm{N}$ & DF & G & $\mathrm{r}$ & + & & & & & & \\
\hline 344 & Parthenocissus inserta (A. Kern.) Fritsch & $\mathrm{CA}$ & NTH & $\mathrm{H}$ & $\mathrm{r}$ & & & & & & & \\
\hline 345 & Peucedanum palustre (L.) Moench & $\mathrm{N}$ & $\mathrm{R}$ & $\mathrm{H}$ & $\mathrm{s}$ & + & & & & & + & \\
\hline 346 & Phalaris arundinacea L. [Phalaroides arundinacea (L.) Rausch.] & $\mathrm{N}$ & LTH & $\mathrm{H}$ & $\mathrm{c}$ & + & & + & + & + & + & + \\
\hline 347 & Phegopteris connectilis (Michx.) Watt [Thelypteris phegopteris (L.) Sloss.] & $\mathrm{N}$ & $\mathrm{CF}$ & $\mathrm{H}$ & $\mathrm{r}$ & + & & & & & & \\
\hline 348 & Phleum pratense L. s.l. & A & M & $\mathrm{H}$ & $\mathrm{s}$ & + & & & & & & \\
\hline 349 & Phragmites australis (Cav.) Trin. et Steudel & $\mathrm{N}$ & $\mathrm{R}$ & Hy & $\mathrm{c}$ & + & & + & + & & + & + \\
\hline 350 & Picea abies (L.) H. Karst. s.l. & $\mathrm{N}$ & $\mathrm{CF}$ & MF & $\mathrm{c}$ & + & & & & + & + & + \\
\hline 351 & Pimpinella major (L.) Huds. & $\mathrm{CA}$ & M & $\mathrm{H}$ & $r$ & + & $\mathrm{CR}$ & & & & & \\
\hline 352 & Pimpinella saxifraga $\mathrm{L}$. & A & $\mathrm{X}$ & $\mathrm{H}$ & $\mathrm{s}$ & + & & & & & + & \\
\hline 353 & Pinus sylvestris L. & $\mathrm{N}$ & $\mathrm{CF}$ & MF & $\mathrm{c}$ & + & & + & + & + & + & + \\
\hline 354 & Plantago intermedia DC [P. major ssp. intermedia (DC.) Arcang.] & $\mathrm{N}$ & Ss & $\mathrm{T}$ & $\mathrm{r}$ & & & & & & & \\
\hline 355 & Plantago lanceolata $\mathrm{L}$. & A & M & $\mathrm{H}$ & $\mathrm{s}$ & + & & & & & & \\
\hline 356 & Plantago major L. s. str. & A & M & $\mathrm{H}$ & $\mathrm{f}$ & + & & & & + & & \\
\hline 357 & Plantago maritima $\mathrm{L}$. & $\mathrm{N}$ & $\mathrm{H}$ & $\mathrm{H}$ & $\mathrm{c}$ & + & & + & + & & + & + \\
\hline 358 & Plantago winteri Wirtg. [P. major ssp. winteri (Wirtg.) W. Ludwig] & $\mathrm{N}$ & $\mathrm{H}$ & $\mathrm{H}$ & $\mathrm{c}$ & + & & & + & + & + & + \\
\hline 359 & Platanthera bifolia (L.) Rich. & $\mathrm{N}$ & $\mathrm{DF}$ & $\mathrm{G}$ & $\mathrm{s}$ & + & & & & + & + & \\
\hline 360 & Poa angustifolia $\mathrm{L}$. & $\mathrm{N}$ & $\mathrm{X}$ & $\mathrm{H}$ & $\mathrm{s}$ & + & & & & & & \\
\hline
\end{tabular}




$\begin{array}{llllllllllllllllllllllllllll}+ & + & + & + & + & + & + & + & + & + & + & + & & + & + & + & + & + & + & + & + & + & + & + & + & + \\ + & + & + & + & + & + & + & + & + & + & + & + & + & + & + & + & + & + & + & + & + & + & + & + & + & +\end{array}$

$+++++\quad+\quad+\quad+\quad+\quad+\quad+\quad+\quad+\quad++++$




\begin{tabular}{|c|c|c|c|c|c|c|c|c|c|c|c|c|}
\hline 1 & 2 & 3 & 4 & 5 & 6 & 78 & 9 & 10 & 11 & 12 & 13 & 14 \\
\hline 361 & Poа аппиа $\mathrm{L}$. & $\mathrm{A}$ & SR & $\mathrm{T}$ & $\mathrm{s}$ & + & & & & & & \\
\hline 362 & Poa compressa L. ssp. compressa & $\mathrm{N}$ & SR & $\mathrm{H}$ & $\mathrm{r}$ & + & & & & & & \\
\hline 363 & Poa nemoralis L. & $\mathrm{N}$ & $\mathrm{DF}$ & $\mathrm{H}$ & $\mathrm{f}$ & + & & & & + & + & \\
\hline 364 & Poa palustris $\mathrm{L}$. & $\mathrm{N}$ & M & $\mathrm{H}$ & $\mathrm{s}$ & + & & & & & & \\
\hline 365 & Poa pratensis L.s. str. & A & $\mathrm{M}$ & $\mathrm{H}$ & $\mathrm{s}$ & + & & & & & & \\
\hline 366 & Poa subcaerulea Sm. [Poa humilis Ehrh. ex Hoffm.] & $\mathrm{N}$ & M & $\mathrm{H}$ & $\mathrm{s}$ & + & & & & & & \\
\hline 367 & Poa trivialis $\mathrm{L}$. & $\mathrm{N}$ & M & $\mathrm{H}$ & $\mathrm{s}$ & + & & & & & + & \\
\hline 368 & Polygonatum multiflorum (L.) All. & $\mathrm{N}$ & DF & G & $\mathrm{r}$ & + & & & & & & \\
\hline 369 & Polygonatum odoratum (Mill.) Druce & $\mathrm{N}$ & $\mathrm{x}$ & $\mathrm{G}$ & $\mathrm{s}$ & + & & & & & & \\
\hline 370 & $\begin{array}{l}\text { Polygonum arenastrum Boreau [P. aequale Lindm., P. aviculare L. ssp. microspermum (Jord. } \\
\text { ex Boreau) Berher p.p.] }\end{array}$ & A & SR & $\mathrm{T}$ & $\mathrm{r}$ & & & & & & & \\
\hline \multirow[t]{2}{*}{371} & Polygonum aviculare L. ssp. aviculare $[P$. heterophyllum Lindm.] & A & SR & $\mathrm{T}$ & $\mathrm{s}$ & + & & & & & & \\
\hline & Polygonum aviculare L. ssp. boreale (Lange) Karlsson [P. aviculare L. var. littorale auct.] & $\mathrm{N}$ & $\mathrm{H}$ & $\mathrm{T}$ & $\mathrm{s}$ & + & & + & + & & & \\
\hline 372 & Polygonum calcatum Lindm. [P. aviculare L. ssp. microspermum (Jord. ex Boreau) Berher p.p.] & A & SR & $\mathrm{T}$ & $\mathrm{r}$ & & & & & & & \\
\hline 373 & Polygonum hydropiper L. [Persicaria hydropiper (L.) Spach] & A & LTH & $\mathrm{T}$ & $\mathrm{r}$ & + & & & & & & \\
\hline 374 & Polygonum neglectum Besser [P. aviculare L. ssp. neglectum (Besser) Arcang.] & $\mathrm{N}$ & SR & $\mathrm{T}$ & $\mathrm{rr}$ & + & & & & & & \\
\hline 375 & $\begin{array}{l}\text { Polygonum nodosum Pers. }[\text { P. lapathifolium L. ssp. lapathifolium, Persicaria lapathifolia (L.) } \\
\text { Gray ssp. lapathifolia }]\end{array}$ & A & SS & $\mathrm{T}$ & $\mathrm{rr}$ & + & & & & & & \\
\hline 376 & Polygonum oxyspermum C. A. Mey. \& Bunge ex Ledeb. & $\mathrm{N}$ & $\mathrm{H}$ & $\mathrm{T}$ & $\mathrm{r}$ & & $\mathrm{CR}$ & + & + & & + & \\
\hline 377 & Polygonum persicaria L. [Persicaria maculosa Gray] & A & SS & $\mathrm{T}$ & $\mathrm{r}$ & + & & & & & & \\
\hline 378 & $\begin{array}{l}\text { Polygonum tomentosum Schrank [P. lapathifolium L. ssp. pallidum (With.) Fr., Persicaria } \\
\text { lapathifolia ssp. pallida (With.) S. Ekman \& T. Knutsson] }\end{array}$ & A & SS & $\mathrm{T}$ & $\mathrm{r}$ & + & & & & & & \\
\hline 379 & Polypodium vulgare $\mathrm{L}$. & $\mathrm{N}$ & $\mathrm{Ch}$ & $\mathrm{H}$ & $\mathrm{c}$ & + & & & + & + & + & + \\
\hline 380 & Populus tremula $\mathrm{L}$. & $\mathrm{N}$ & $\mathrm{DF}$ & MF & $\mathrm{f}$ & + & & & & & + & \\
\hline 381 & Potamogeton filiformis Pers. [Stuckenia filiformis (Pers.) Börner] & $\mathrm{N}$ & $\mathrm{W}$ & Hy & $\mathrm{f}$ & + & & + & + & + & + & \\
\hline 382 & Potamogeton pectinatus L. [Stuckenia pectinata (L.) Börner] & $\mathrm{N}$ & W & $\mathrm{Hy}$ & $\mathrm{s}$ & + & & & & + & & \\
\hline 383 & Potamogeton perfoliatus L. & $\mathrm{N}$ & W & Hy & $\mathrm{c}$ & + & & + & + & + & + & \\
\hline 384 & Potamogeton pusillus L. & $\mathrm{N}$ & W & Hy & $\mathrm{r}$ & + & & & & & & \\
\hline 385 & Potentilla anserina L. ssp. anserina [Argentina anserina (L.) Rydb.] & $\mathrm{N}$ & M & $\mathrm{H}$ & $f$ & + & & & + & + & + & \\
\hline 386 & Potentilla argentea L. s. str. & A & $\mathrm{X}$ & $\mathrm{H}$ & $\mathrm{s}$ & & & & & & + & \\
\hline 387 & Potentilla crantzii (Crantz) Beck. ex Fritsch & $\mathrm{N}$ & $\mathrm{x}$ & $\mathrm{H}$ & $\mathrm{r}$ & + & & & & & & \\
\hline 388 & Potentilla erecta (L.) Raeusch. & $\mathrm{N}$ & M & $\mathrm{H}$ & $\mathrm{f}$ & + & & & & + & + & \\
\hline 389 & Primula veris $\mathrm{L}$. & $\mathrm{N}$ & $\mathrm{X}$ & $\mathrm{H}$ & $\mathrm{s}$ & + & & & & & & \\
\hline 390 & Prunella vulgaris L. & $\mathrm{N}$ & M & $\mathrm{H}$ & $\mathrm{s}$ & + & & & & & + & \\
\hline 391 & Pteridium aquilinum (L.) Kuhn ssp. pinetorum (C. N. Page \& R. R. Mill) J. A. Thomson & $\mathrm{N}$ & $\mathrm{CF}$ & G & $\mathrm{f}$ & + & & & & + & + & \\
\hline 392 & $\begin{array}{l}\text { Puccinellia capillaris (Lilj.) Jansen [P. distans (L.) Parl. ssp. borealis Holmberg, P. retroflexa } \\
\text { W. E. Hughes] }\end{array}$ & $\mathrm{N}$ & $\mathrm{H}$ & $\mathrm{H}$ & $\mathrm{s}$ & + & & & + & + & + & \\
\hline 393 & Pyrola chlorantha Sw. & $\mathrm{N}$ & $\mathrm{CF}$ & $\mathrm{H}$ & $\mathrm{s}$ & + & & & & + & & \\
\hline 394 & Pyrola media $\mathrm{Sw}$. & $\mathrm{N}$ & $\mathrm{CF}$ & $\mathrm{H}$ & $\mathrm{r}$ & + & NT & & & & & \\
\hline 395 & Pyrola minor $\mathrm{L}$. & $\mathrm{N}$ & $\mathrm{CF}$ & $\mathrm{H}$ & $\mathrm{s}$ & + & & & & + & + & \\
\hline \multirow[t]{2}{*}{396} & $\begin{array}{l}\text { Pyrola rotundifolia L. ssp. norvegica (Knaben) Hämet-Ahti [P. r. ssp. maritima (Kenyon) E. F. } \\
\text { Warburg] }\end{array}$ & $\mathrm{N}$ & $\mathrm{CF}$ & $\mathrm{H}$ & $\mathrm{rr}$ & ++ & & & & & & \\
\hline & Pyrola rotundifolia L. ssp. rotundifolia & $\mathrm{N}$ & $\mathrm{CF}$ & $\mathrm{H}$ & $\mathrm{r}$ & + & & & & & & \\
\hline 397 & Quercus robur $\mathrm{L}$. & $\mathrm{N}$ & $\mathrm{DF}$ & MF & $\mathrm{r}$ & + & & & & & & \\
\hline 398 & Ranunculus acris L. ssp. acris & $\mathrm{N}$ & M & $\mathrm{H}$ & $\mathrm{s}$ & + & & & & & & \\
\hline 399 & Ranunculus auricomus $\mathrm{L}$. & $\mathrm{N}$ & M & $\mathrm{H}$ & $\mathrm{s}$ & & & & & + & + & \\
\hline 400 & Ranunculus bulbosus L. & A & $\mathrm{x}$ & $\mathrm{H}$ & $\mathrm{r}$ & + & & & & & & \\
\hline 401 & Ranunculus flammula $\mathrm{L}$. & $\mathrm{N}$ & $\mathrm{R}$ & $\mathrm{H}$ & $\mathrm{r}$ & + & & & & & & \\
\hline 402 & Ranunculus polyanthemos L. ssp. polyanthemos & A & $\mathrm{x}$ & $\mathrm{H}$ & $\mathrm{s}$ & + & & & & & & \\
\hline 403 & Ranunculus repens $\mathrm{L}$. & $\mathrm{N}$ & M & $\mathrm{H}$ & $\mathrm{f}$ & + & & & & & + & \\
\hline 404 & Raphanus raphanistrum $\mathrm{L}$. & A & SS & $\mathrm{T}$ & $\mathrm{rr}$ & + & & & & & & \\
\hline 405 & Raphanus sativus L. & $\mathrm{CA}$ & $\mathrm{C} / \mathrm{SR}$ & $\mathrm{T}$ & $\mathrm{rr}$ & & & & & & & \\
\hline 406 & Rhinanthus minor L. ssp. minor & A & M & $\mathrm{T}$ & $\mathrm{r}$ & + & & & & & & \\
\hline 407 & Ribes alpinum $\mathrm{L}$. & $\mathrm{N}$ & DF & $\mathrm{NF}$ & $\mathrm{f}$ & + & & & & + & + & \\
\hline 408 & Ribes nigrum $\mathrm{L}$. & $\mathrm{N}$ & $\mathrm{DF}$ & $\mathrm{NF}$ & $\mathrm{s}$ & + & & & & & & \\
\hline 409 & Ribes spicatum E.Robson s.1. & $\mathrm{N}$ & $\mathrm{DF}$ & $\mathrm{NF}$ & $\mathrm{s}$ & + & & & & & & \\
\hline 410 & Ribes uva-crispa L. & EA & $\mathrm{DF}$ & $\mathrm{NF}$ & $\mathrm{r}$ & + & & & & & & \\
\hline 411 & Rorippa palustris (L.) Besser & $\mathrm{N}$ & LTH & $\mathrm{T}$ & $\mathrm{rr}$ & + & & & & & & \\
\hline 412 & Rosa caesia $\mathrm{Sm}$. [R. dumalis Bechst. ssp. coriifolia (Fr.) auct.] & $\mathrm{N}$ & $\mathrm{X}$ & NF & $\mathrm{s}$ & + & & & & & + & \\
\hline 413 & Rosa canina L. s. str. & $\mathrm{N}$ & $\mathrm{x}$ & $\mathrm{NF}$ & s & . & $\mathrm{CR}$ & & & & & \\
\hline 414 & Rosa corymbifera Borkh. [R. canina L. ssp. dumetorum (Thuill.) auct.] & $\mathrm{N}$ & $\mathrm{x}$ & $\mathrm{NF}$ & $\mathrm{r}$ & + & $\mathrm{DD}$ & & & & & \\
\hline 415 & Rosa dumalis Bechst. s. str. & $\mathrm{N}$ & $\mathrm{x}$ & $\mathrm{NF}$ & s & + & & & & & & \\
\hline 416 & Rosa majalis Herrm. [R. cinnamomea L.] & $\mathrm{N}$ & $\mathrm{x}$ & $\mathrm{NF}$ & $\mathrm{rr}$ & + & & & & & & \\
\hline 417 & Rosa mollis Sm. (R. villosa L.) & $\mathrm{N}$ & $\mathrm{x}$ & $\mathrm{NF}$ & $\mathrm{rr}$ & + & & & & & & \\
\hline 418 & Rosa spinosissima L. $[R$. pimpinellifolia $\mathrm{L}]$. & $\mathrm{CA}$ & $\mathrm{C} / \mathrm{SR}$ & NF & $\mathrm{r}$ & + & & & & & & \\
\hline
\end{tabular}




\begin{tabular}{|c|c|c|c|c|c|c|c|c|c|c|c|}
\hline 1 & 2 & 3 & 4 & 5 & 6 & $\begin{array}{lll}78 & 9\end{array}$ & 10 & 11 & 12 & 13 & 14 \\
\hline 419 & Rosa 'Splendens' [incl. $R . \quad \times a l b a$ L.] & $\mathrm{CA}$ & $\mathrm{C} / \mathrm{SR}$ & $\mathrm{NF}$ & $\mathrm{r}$ & + & & & & & \\
\hline 420 & Rubus chamaemorus L. & $\mathrm{N}$ & $\mathrm{CF}$ & $\mathrm{H}$ & $\mathrm{rr}$ & & & & & & \\
\hline 421 & Rubus idaeus L. & $\mathrm{N}$ & NTH & NF & $\mathrm{c}$ & + & & & + & + & + \\
\hline 422 & Rubus saxatilis L. & $\mathrm{N}$ & $\mathrm{CF}$ & $\mathrm{H}$ & $\mathrm{f}$ & + & & & & + & \\
\hline 423 & Rumex acetosa L. ssp. acetosa & $\mathrm{N}$ & M & $\mathrm{H}$ & $\mathrm{s}$ & + & & & + & & \\
\hline 424 & Rumex acetosella L. ssp. acetosella & $\mathrm{N}$ & $\mathrm{x}$ & $\mathrm{H}$ & $\mathrm{s}$ & + & & & & & \\
\hline 425 & Rumex crispus L. s.l. & $\mathrm{N}$ & M & $\mathrm{H}$ & $\mathrm{c}$ & + & & + & + & + & + \\
\hline 426 & Rumex longifolius DC. & A & SR & $\mathrm{H}$ & $\mathrm{r}$ & + & & & & + & \\
\hline 427 & Rumex tenuifolius (Wallr.) A. Löve [R. acetosella L. ssp. tenuifolius (Wallr.) O. Schwarz] & $\mathrm{N}$ & $\mathrm{X}$ & $\mathrm{H}$ & $\mathrm{c}$ & + & & + & + & + & + \\
\hline 428 & Sagina nodosa (L.) Fenzl ssp. nodosa & $\mathrm{N}$ & $\mathrm{Ch}$ & $\mathrm{C}$ & $\mathrm{r}$ & + & & & & & \\
\hline 429 & Sagina procumbens L. & $\mathrm{N}$ & SR & $\mathrm{T}$ & $\mathrm{c}$ & + & + & & + & + & + \\
\hline 430 & Salix aurita $\mathrm{L}$. & $\mathrm{N}$ & $\mathrm{DF}$ & $\mathrm{NF}$ & $\mathrm{r}$ & + & & & & & \\
\hline 431 & Salix caprea $\mathrm{L}$. & $\mathrm{N}$ & $\mathrm{DF}$ & MF & $\mathrm{f}$ & + & & & + & + & \\
\hline 432 & Salix cinerea L. & $\mathrm{N}$ & DF & NF & $\mathrm{s}$ & + & & & & & \\
\hline 433 & Salix pentandra L. & $\mathrm{N}$ & $\mathrm{DF}$ & MF & $\mathrm{r}$ & + & & & & & \\
\hline 434 & Salix phylicifolia $\mathrm{L}$. & $\mathrm{N}$ & DF & $\mathrm{NF}$ & $\mathrm{rr}$ & + & & & & & \\
\hline 435 & Salix starkeana Willd. ssp. starkeana & $\mathrm{N}$ & $\mathrm{DF}$ & $\mathrm{NF}$ & $\mathrm{rr}$ & + & & & & & \\
\hline 436 & Sambucus racemosa L. & EA & NTH & $\mathrm{NF}$ & $\mathrm{r}$ & + & & & & & \\
\hline 437 & Saponaria officinalis $\mathrm{L}$. & EA & SR & $\mathrm{H}$ & $\mathrm{r}$ & + & & & & & \\
\hline 438 & Schoenoplectus tabernaemontani (C. C. Gmel.) Palla & $\mathrm{N}$ & $\mathrm{R}$ & Hy & $\mathrm{s}$ & + & & + & & & \\
\hline 439 & Scleranthus annuus L. ssp. annuus & A & SS & $\mathrm{T}$ & $\mathrm{rr}$ & + & & & & & \\
\hline 440 & Scleranthus polycarpos L. [S. annus ssp. polycarpos (L.) Thell.] & A & $\mathrm{X}$ & $\mathrm{T}$ & $\mathrm{r}$ & + & & & & & \\
\hline 441 & Scrophularia nodosa L. & $\mathrm{N}$ & DF & $\mathrm{H}$ & $\mathrm{f}$ & + & & & & + & \\
\hline 442 & Scutellaria galericulata $\mathrm{L}$. & $\mathrm{N}$ & $\mathrm{R}$ & $\mathrm{H}$ & $\mathrm{s}$ & + & & & + & + & \\
\hline 443 & Scutellaria hastifolia L. & $\mathrm{N}$ & $\mathrm{x}$ & $\mathrm{H}$ & $\mathrm{r}$ & + & & & & & \\
\hline 444 & Sedum acre L. & $\mathrm{N}$ & $\mathrm{x}$ & $\mathrm{C}$ & $\mathrm{c}$ & + & & + & + & + & + \\
\hline 445 & Sedum album L. & $\mathrm{N}$ & $\mathrm{x}$ & $\mathrm{C}$ & $\mathrm{rr}$ & + & & & & & \\
\hline 446 & $\begin{array}{l}\text { Sedum maximum (L.) Hoffm. [S. telephium L. ssp. maximum (L.) Schinz \& Thell., } \\
\text { Hylotelephium maximum (L.) Holub] }\end{array}$ & $\mathrm{N}$ & $\mathrm{x}$ & G & $\mathrm{c}$ & + & & + & + & + & + \\
\hline 447 & Sedum spurium M.Bieb. [Phedimus spurius (M. Bieb.) 't Hart] & EA & $\mathrm{x}$ & $\mathrm{C}$ & $\mathrm{r}$ & + & & & & & \\
\hline 448 & Senecio sylvaticus L. & $\mathrm{N}$ & NTH & $\mathrm{T}$ & $\mathrm{c}$ & + & & + & + & + & + \\
\hline 449 & Senecio viscosus L. & $\mathrm{EA}$ & SR & $\mathrm{T}$ & $\mathrm{r}$ & + & & & & & \\
\hline 450 & Senecio vulgaris $\mathrm{L}$. & A & Ss & $\mathrm{T}$ & $\mathrm{r}$ & + & & + & & & \\
\hline 451 & Silene nutans L. & $\mathrm{N}$ & $\mathrm{X}$ & $\mathrm{H}$ & $\mathrm{r}$ & + & & & & & \\
\hline 452 & Silene viscosa (L.) Pers. & $\mathrm{N}$ & SS & $\mathrm{H}$ & $\mathrm{rr}$ & + & & & & & \\
\hline 453 & Silene vulgaris (Moenh) Garcke var. littoralis (Rupr.) Jalas & $\mathrm{N}$ & LTH & $\mathrm{C}$ & $\mathrm{s}$ & + & + & & + & + & \\
\hline 454 & Sisymbrium officinale (L.) Scop. & A & SR & $\mathrm{T}$ & $\mathrm{r}$ & + & & & & & \\
\hline 455 & Solanum dulcamara $\mathrm{L}$. & $\mathrm{N}$ & $\mathrm{DF}$ & $\mathrm{NF}$ & $\mathrm{s}$ & + & & & & & \\
\hline 456 & Solanum nigrum L. s.l. & A & Ss & $\mathrm{T}$ & $\mathrm{rr}$ & + & & & & & \\
\hline 457 & Solidago virgaurea L. ssp. virgaurea & $\mathrm{N}$ & $\mathrm{CF}$ & $\mathrm{H}$ & $\mathrm{rr}$ & + & & & & & \\
\hline 458 & Sonchus arvensis L. var. arvensis & A & ss & $\mathrm{H}$ & $\mathrm{s}$ & + & & & & + & \\
\hline & Sonchus arvensis L. var. maritimus Wahlb. & $\mathrm{N}$ & LTH & $\mathrm{H}$ & $\mathrm{c}$ & + & & + & + & + & + \\
\hline 459 & Sonchus asper (L.) Hill. & A & ss & $\mathrm{T}$ & $\mathrm{r}$ & + & & & & & \\
\hline 460 & Sonchus oleraceus L. & A & SS & $\mathrm{T}$ & $\mathrm{r}$ & + & & & & & \\
\hline 461 & Sorbus aucuparia L. ssp. aucuparia & $\mathrm{N}$ & $\mathrm{F}$ & MF & $\mathrm{c}$ & + & + & + & + & + & + \\
\hline 462 & Sorbus intermedia (Ehrh.) Pers. [S. suecica (L.) Krok. et Almq.] & $\mathrm{N}$ & DF & MF & $\mathrm{rr}$ & $+\mathrm{VU}$ & & & & & \\
\hline 463 & Sorbushybrida L. [S. fennica (Kalm) Fr., Hedlundia hybrida auct.] & $\mathrm{N}$ & DF & MF & $\mathrm{rr}$ & + & & & & & \\
\hline 464 & Sparganium natans L. [S. minimum Wallr.] & $\mathrm{N}$ & $\mathrm{W}$ & Hy & $\mathrm{rr}$ & + & & & & & \\
\hline 465 & Spergula arvensis L. ssp. arvensis & A & SS & $\mathrm{T}$ & $\mathrm{r}$ & + & & & & & \\
\hline 466 & Spergula morisonii Boreau & $\mathrm{N}$ & $\mathrm{X}$ & $\mathrm{T}$ & $\mathrm{s}$ & + & & & + & & + \\
\hline 467 & Spergularia rubra (L.) J. Presl et C. Presl & A & SR & $\mathrm{H}$ & $\mathrm{r}$ & + & & & & & \\
\hline 468 & Spergularia salina J. Presl et C. Presl [S. marina (L.) Griseb.] & $\mathrm{N}$ & $\mathrm{H}$ & $\mathrm{H}$ & $\mathrm{s}$ & + & + & + & & & \\
\hline 469 & Spiraea $\times$ billiardii Hérincq & EA & NTH & $\mathrm{NF}$ & $\mathrm{r}$ & + & & & & & \\
\hline 470 & Stachys palustris $\mathrm{L}$. & $\mathrm{N}$ & LTH & $\mathrm{G}$ & $\mathrm{f}$ & + & & + & + & + & \\
\hline 471 & Stellaria graminea $\mathrm{L}$. & $\mathrm{N}$ & M & $\mathrm{H}$ & $\mathrm{f}$ & + & & & & + & \\
\hline 472 & Stellaria holostea L. & $\mathrm{N}$ & DF & $\mathrm{C}$ & $\mathrm{r}$ & + & & & & & \\
\hline 473 & Stellaria longifolia Willd. & $\mathrm{N}$ & $\mathrm{CF}$ & $\mathrm{H}$ & $\mathrm{r}$ & + & & & & & \\
\hline 474 & Stellaria media (L.) Vill. & $\mathrm{N}$ & SS & $\mathrm{T}$ & $\mathrm{s}$ & + & & & & & \\
\hline 475 & Stellaria palustris Retz. & $\mathrm{N}$ & M & $\mathrm{H}$ & $\mathrm{r}$ & + & & & & & \\
\hline 476 & Tanacetum vulgare $\mathrm{L}$. & $\mathrm{N}$ & LTH & $\mathrm{H}$ & $\mathrm{c}$ & + & + & + & + & + & + \\
\hline 477 & Taraxacum balticum Dahlst. & $\mathrm{N}$ & LTH & $\mathrm{H}$ & $\mathrm{s}$ & + & + & + & & + & \\
\hline 478 & Taraxacum dahlstedtii H. Lindb. & $\mathrm{N}$ & LTH & $\mathrm{H}$ & $\mathrm{r}$ & + & & & & & \\
\hline
\end{tabular}




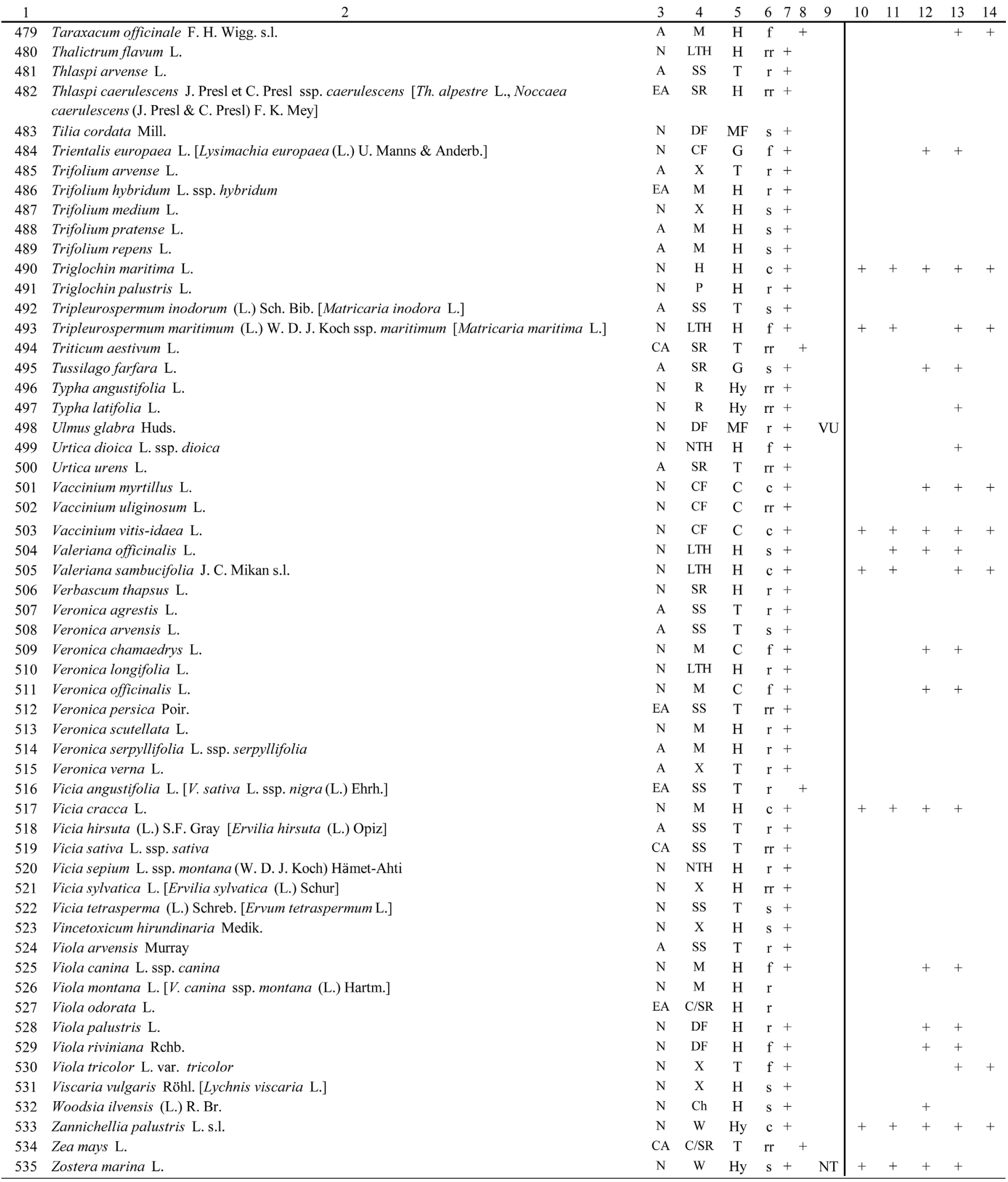

Explanations: 1 - subsequent number of species; 2 - names of taxa; 3 - origin of taxa (for Regio aboënsis): $\mathrm{N}$ - native, A - archaeophyte, EA - established alien, CA - casual alien; 4 - socioeco-logical group (comp. Tab. 1): DF - Deciduous forests, CF - Coniferous forests, LTH - Littoral tall herbs, $\mathrm{H}$ - Halophytes, $\mathrm{P}$ - Peats and mires, $\mathrm{R}$ - reeds, $\mathrm{W}$ - water, $\mathrm{F}$ - general forest, $\mathrm{Ch}$ - chasmophytes, $\mathrm{M}$ - meadows, pastures and heathland, X - Xerothermophilous swards, forest edges and thickets, NTH - Nitrophilous tall herbs and thickets connected with forests, SS synanthropic segetal, SR - synanthropic ruderal, C/SR - cultivated and locally escaping on ruderal sites; 5 - life forms: MF - megaphanerophytes, 
Appendix 2. Specification of cultivated vascular plants of Seili archipelago

\begin{tabular}{|c|c|c|c|c|c|c|c|c|c|c|c|c|c|c|c|c|c|}
\hline No & Taxon & 1 & 23 & $\begin{array}{c}4 \\
\mathrm{C}_{2}\end{array}$ & $\begin{array}{c}5 \\
\mathrm{D}_{2}\end{array}$ & $\begin{array}{r}6 \\
\mathrm{D}_{3}\end{array}$ & $\begin{array}{cc}7 & 8 \\
\mathrm{D}_{4} & \mathrm{D}_{5}\end{array}$ & $\begin{array}{c}9 \\
\mathrm{E}_{1}\end{array}$ & $\begin{array}{l}10 \\
E_{2}\end{array}$ & $\begin{array}{l}11 \\
E_{3}\end{array}$ & $\begin{array}{l}12 \\
\mathrm{E}_{4}\end{array}$ & $\begin{array}{ll}13 & 1 \\
F_{1} & \mathrm{I}\end{array}$ & $\begin{array}{ll}14 & 15 \\
\mathrm{~F}_{2} & \mathrm{~F}_{3}\end{array}$ & $\begin{array}{ll}16 & 17 \\
\mathrm{~F}_{4} & \mathrm{~F}_{5}\end{array}$ & 18 & 19 & 20 \\
\hline 1 & Abies alba Mill. & & & & & & & & & & & & & & 0 & + & \\
\hline 2 & Abies grandis (Dougl. ex D.Don.) Lindl. & & & & & & & & + & & & & & & 1 & & + \\
\hline 3 & Abies sibirica Ledeb. & & + & & & & & & + & & & & & & 1 & & \\
\hline 4 & Allium cepa $\mathrm{L}$. & & + & & & + & & & & + & & & & & 2 & & \\
\hline 5 & Allium porrum L. & & + & & & + & & & & + & & & & & 2 & & \\
\hline 6 & Allium sativum L. & & + & & & + & & & + & & & & & & 2 & & \\
\hline 7 & Anthriscus cerefolium (L.) Hoffm. & & & & & + & & & & & & & & & 1 & & + \\
\hline 8 & Apium graveolens $\mathrm{L}$. & & + & & & + & & & & + & & & & & 2 & & \\
\hline 9 & Artemisia dracunculus L. & & & & & & & & & + & & & & & 1 & & + \\
\hline 10 & Bergenia crassifolia (L.) Fritsch & & + & & + & & & & + & & & & & & 2 & & \\
\hline 11 & $\begin{array}{l}\text { Beta vulgaris L. ssp. rapacea (Koch) } \\
\text { Döll }\end{array}$ & & + & & & + & & & & + & + & & & & 3 & & \\
\hline 12 & $\begin{array}{l}\text { Betula fontinalis Sarg. (=B. occidentalis } \\
\text { Hook.) }\end{array}$ & & & & & & & & & & + & & & + & 2 & & + \\
\hline 13 & Betula japonica Sieb. & & & & & & & & & & + & & & + & 2 & & + \\
\hline 14 & Betula papyrifera Marshall & & & & & & & & & & + & & & + & 2 & & + \\
\hline 15 & $\begin{array}{l}\text { Brassica cretica Lam. ssp. botrytis (L.) } \\
\text { O. Schwarz }\end{array}$ & & + & & & + & & & & & & & & & 1 & & \\
\hline 16 & Brassica oleracea L. s.1. & & + & & & + & & & & & & & & & 1 & & \\
\hline 17 & Calendula officinalis $\mathrm{L}$. & & + & & & + & & & + & + & & & & & 3 & & \\
\hline 18 & Caragana arborescens Lam. & & + & & + & & & & & & & & + & + & 3 & & \\
\hline 19 & $\begin{array}{l}\text { Centaurea macrocephala Muss. Puschk. } \\
\text { ex Willd. }\end{array}$ & & + & & & & & & + & & & & & & 1 & & \\
\hline 20 & Cerastium tomentosum $\mathrm{L}$. & & + & & & + & & & + & + & & + & & & 4 & & \\
\hline 21 & $\begin{array}{l}\text { Cerasus avium (L.) Moench (=Prunus } \\
\text { avium (L.) L.) }\end{array}$ & & + & + & + & & & + & + & & & & & & 4 & & \\
\hline 22 & $\begin{array}{l}\text { Cerasus vulgaris Mill. (=Prunus cerasus } \\
\text { L.) }\end{array}$ & + & & & + & + & & & + & + & + & + & & & 6 & & \\
\hline 23 & $\begin{array}{l}\text { Chaenomeles japonica (Thunb.) Lindl. } \\
\text { ex Spach }\end{array}$ & & + & & + & & & & & & & & & & 1 & & \\
\hline 24 & Cucumis sativus $\mathrm{L}$. & & & & & + & & & & + & + & & & & 3 & & \\
\hline 25 & Cucurbita pepo L. & & & & & + & & & & + & & & & & 2 & & \\
\hline 26 & Dahlia hybrida hort. & & + & & & + & & & & & + & & & & 2 & & \\
\hline 27 & $\begin{array}{l}\text { Daucus carota L. ssp. sativus (Hoffm.) } \\
\text { Arcang. }\end{array}$ & & + & & & + & & & & + & & & & & 2 & & \\
\hline 28 & Delphinium $\times$ cultorum Voss & & + & & & & & & & + & & & & & 1 & & \\
\hline 29 & Dianthus barbatus L. & & + & & + & & & & & & + & & & & 2 & & \\
\hline 30 & Dicentra formosa (Haw.) Walp. & & & & & + & & & + & + & & & & & 3 & & + \\
\hline 31 & Eleagnus commutata Bernh. & & + & & & & & & & & & & + & & 1 & & \\
\hline 32 & Fragaria $\times$ ananassa (Weston) Rozier & & + & + & + & + & & & + & + & + & & & + & 7 & & \\
\hline 33 & Helianthus tuberosus L. & & + & & & + & & & & & & & & & 1 & & \\
\hline 34 & Helleborus L. spec. & & & & & & & & + & & + & & & & 2 & & \\
\hline 35 & Hemerocallis fulva (L.) L. & & & & + & + & & & + & + & + & & & & 5 & & + \\
\hline 36 & Hemerocallis lilioasphodelus L. & & & & & & & & + & + & & & & & 2 & & + \\
\hline 37 & $\begin{array}{l}\text { Heracleum mantegazzianum Somier et } \\
\text { Levier. }\end{array}$ & & + & & & & & & + & & & & & & 1 & & \\
\hline 38 & Hyssopus officinalis L. & + & & & & & & & & & & & & & 0 & & + \\
\hline 39 & Iris germanica L. & & + & & + & & & & + & + & & & & + & 4 & & \\
\hline 40 & Lactuca sativa $\mathrm{L}$. & & + & & & + & & & & + & & & & & 2 & & \\
\hline 41 & Lilium bulbiferum $\mathrm{L}$. & & + & & & & + & & & + & + & + & & & 4 & & \\
\hline 42 & Linum usitatissimum L. & & + & & & & & & & + & & & & & 1 & & \\
\hline 43 & Lycopersicon esculentum Mill. & & + & & & + & & & & + & & & & & 2 & & \\
\hline 44 & Melissa officinalis $\mathrm{L}$. & & + & & + & & & & & & & & & & 1 & & \\
\hline 45 & Muscari botryoides (L.) Mill. & & & & & + & + & & & + & & & & & 3 & & + \\
\hline 46 & Narcissus poeticus L. & & & & & + & + & & & + & & & + & & 4 & & + \\
\hline 47 & $\begin{array}{l}\text { Ornithogallum angustifolium Boreau } \\
(=\text { O. umbellatum } \mathrm{L} .)\end{array}$ & + & & & & + & & & & + & & & & & 2 & & + \\
\hline 48 & Paeonia officinalis L. & & + & & & & & & + & + & & & & & 2 & & \\
\hline 49 & Petroselinum crispum (Mill.) A. W. Hill & & + & & & + & & & & + & + & & & & 3 & & \\
\hline
\end{tabular}




\begin{tabular}{|c|c|c|c|c|c|c|c|c|c|c|c|c|c|c|c|c|c|c|c|c|c|}
\hline No & Taxon & 1 & 2 & 3 & 4 & 5 & 6 & 7 & 8 & 9 & 10 & 11 & 12 & 13 & 14 & 15 & 16 & 17 & 18 & 19 & 20 \\
\hline 50 & Petunia $\times$ atkinsiana D. Don & & + & & & & + & & & & & + & & & & & & & 2 & & \\
\hline 51 & Phaseolus vulgaris L. & & + & & & & + & & & & & + & & & & & & & 2 & & \\
\hline 52 & Philadelphus coronarius L. & & & & & & + & & & & & + & + & & & & & & 3 & & + \\
\hline 53 & Picea engelmannii Parry ex Engelm. & & & & & & & & & & + & & & & & + & & & 2 & & + \\
\hline 54 & Picea glauca (Moench) Voss & & & & & & & & & & & & & & & + & & & 1 & & + \\
\hline 55 & Picea jezoensis (Siebold \& Zucc.) Carr. & & + & & & & & & & & & & & & & + & & & 1 & & \\
\hline 56 & Picea omorica (Panč.) Purk. & & & & & & & & & & & & & & & + & & & 1 & & + \\
\hline 57 & Picea pungens Engelm. & & & & & & & & & & & & & & & + & & & 1 & & + \\
\hline 58 & Picea sitchensis (Bong.) Carr. & & + & & & & & & & & & & & & & + & & & 1 & & \\
\hline 59 & Pisum sativum L. & & + & & & & + & & & & & + & & & & & & & 2 & & \\
\hline 60 & Populus tremula $\times$ P. tremuloides Michx. & & & & & & & & & & & + & & & & & & & 1 & & + \\
\hline 61 & Potentilla fruticosa $\mathrm{L}$. & & & & & & + & & & & & & & & & & & & 1 & & + \\
\hline 62 & Prunus domestica $\mathrm{L}$. & & + & & & + & & & & & + & & + & & & & & & 3 & & \\
\hline 63 & Pseudotsuga menziesii (Mirb.) Franco & & + & & & & & & + & & & & & & & & & & 1 & & \\
\hline 64 & Ptelea trifoliata $\mathrm{L}$. & & + & & & & & & & & & & & & & & & + & 1 & & \\
\hline 65 & Quercus rubra L. & & + & & + & & & & & & & & & & & & & & 1 & & \\
\hline 66 & Rheum rhabarbarum $\mathrm{L}$. & & + & & & & & & & & & + & & & & & & & 1 & & \\
\hline 67 & Salix viminalis L. & & + & & & & & & & & & & & & & & + & & 1 & & \\
\hline 68 & Scilla siberica Haw. & & & & & & + & + & & & + & + & & & & & & & 4 & & + \\
\hline 69 & Solanum tuberosum L. & & + & & & & + & & & & & + & + & & & & & & 3 & & \\
\hline 70 & Solidago gigantea Aiton ssp. serotina & & + & & & & + & & & & & & & & & & & + & 2 & & \\
\hline 71 & $\begin{array}{l}\text { Spiraea chamaedryfolia } \text { L. }(=S . \times \text { arguta } \\
\text { Zabel) }\end{array}$ & & + & & & & + & & & & & & & & & & & & 1 & & \\
\hline 72 & Syringa josikaea Jacq. ex Rchb. & & + & & & & & & & & + & + & & & & & & & 2 & & \\
\hline 73 & Syringa vulgaris L. & & & & & + & + & + & & & + & + & & + & + & & + & & 8 & & + \\
\hline 74 & Tagetes patula $\mathrm{L}$. & & + & & & & + & & & & & + & & & & & & & 2 & & \\
\hline 75 & Taxus baccata L. & & + & NT & & & + & & & & & & & & & & & & 1 & & \\
\hline 76 & Viola $\times$ wittrockiana Gams ex Kappert & & + & & & & + & + & & & & + & & & & & & + & 4 & & \\
\hline
\end{tabular}

Explanations: 1 - species common with data of Lampinen et al. (2016) for square 669:322; 2 - species new for square 669:322; 3 - categories of threat in Finland (Kalliovirta et al. 2010) NT - near threatened; 4-17 - squares, 18 - frequency; 19 - taxa given by Eklund (1946); 20 - herbarium materials and unpublished data submitted by Leila Linnaluoto 